\title{
OpenSHMEM
}

\section{Application Programming Interface}

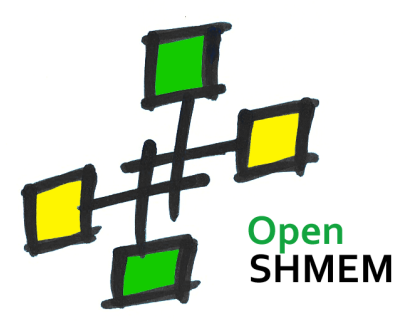

http: //www. openshmem.org/

Version 1.0 FINAL

The comment period for Version 1.0 FINAL ended on January 31, 2012 The comment period for Version 1.1 started on February 1, 2012

\section{Developed by}

- High Performance Computing Tools group at the University of Houston

http://www.cs.uh.edu/ hpctools/

- Extreme Scale Systems Center, Oak Ridge National Laboratory 


\section{Sponsored by}

- U.S. Department of Defense

http://www.defense.gov/

- Oak Ridge National Laboratory

http://www.ornl.gov/

\section{Authors and Collaborators}

- Barbara Chapman, University of Houston

- Tony Curtis, University of Houston

- Ricardo Mauricio, University of Houston

- Swaroop Pophale, University of Houston

- Ram Nanjegowda, University of Houston

- Amrita Banerjee, University of Houston

- Karl Feind, SGI

- Jeff Kuehn, ORNL

- Stephen Poole, ORNL

- Lauren Smith, DoD

\section{Acknowledgements}

The OpenSHMEM development work is supported by the Oak Ridge National Laboratory Extreme Scale System Center.

The following people (listed alphabetically) have contributed ideas, criticisms and suggestions on the openshmem mailing list and in other fora:

Vikas Aggarwal; Brian W. Barrett; Christian Bell; Max Billingsley III; Mark Debbage; Mike Dubman; Dick Foster; Hal Finkel; Roger A. Golliver; Jeff Hammond; Alistair Hart; Tsai-yang Jea; Daniel Kidger; Rishi Khan; David LaFrance-Linden; John Leidel; Alexander Mikheev; Chen Qi; Duncan Roweth; Sameer Shende; Marc Snir; Lawrence Stewart; Keith D. Underwood; Brian Wibecan.

Apologies to people who have contributed but who are not acknowledged here: it is not intentional. 


\section{Contents}

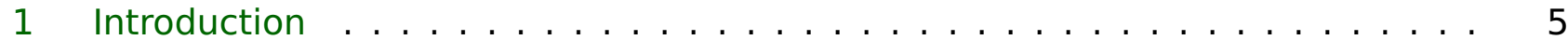

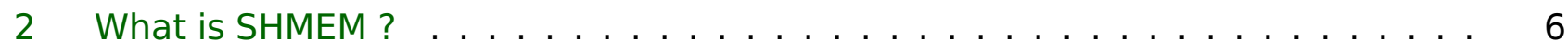

2.1 Partitioned Global Address Space . . . . . . . . . . . . . . . . . . . . . . 6

2.2 SHMEM .......................... 6

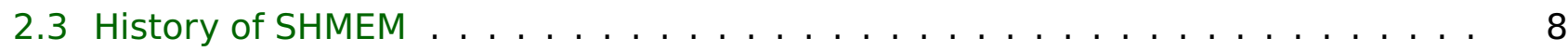

3 The OpenSHMEM Project . . . . . . . . . . . . . . . . . . 10

3.1 What is OpenSHMEM ? . . . . . . . . . . . . . . . . . . . . 10

4 Language Bindings and Conformance . . . . . . . . . . . . . . . . . 11

5 Memory Model . . . . . . . . . . . . . . . . . . . . . . . . . . . . . . . 12

6 Execution Model . . . . . . . . . . . . . . . . . . . . . . . . . . . 13

6.1 Communication Progress . . . . . . . . . . . . . . . . . . . . . . . . 14

6.2 Atomicity Guarantees . . . . . . . . . . . . . . . . . . . . . . . . . 14

7 Undefined Behavior . . . . . . . . . . . . . . . . . . . . . 15

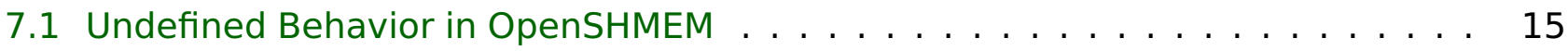

8 Library Routines . . . . . . . . . . . . . . . . . . . . . . . . . . . . . 16

8.1 Initialization Routines . . . . . . . . . . . . . . . . . 16

8.2 Query Routines . . . . . . . . . . . . . . . . . . . . . . . . . 17

8.3 Accessibility Query Routines . . . . . . . . . . . . . . . . . . . . . . . . . . . 19

8.4 Symmetric Heap Routines . . . . . . . . . . . . . . . . . . . . . . . . . . 21

8.5 Remote Pointer Operations . . . . . . . . . . . . . . . . . . . . . . . . 30

8.6 Elemental Put Routines . . . . . . . . . . . . . . . . . . . . . . . . . 31

8.7 Block Data Put Routines . . . . . . . . . . . . . . . . . . . . . 32

8.8 Strided Put Routines . . . . . . . . . . . . . . . . . . . . . 34

8.9 Elemental Data Get Routines . . . . . . . . . . . . . . . . . . . . . 37

8.10 Block Data Get Routines . . . . . . . . . . . . . . . . . . . . . 38

8.11Strided Get Routines . . . . . . . . . . . . . . . . . . . . . . . . 40

8.12Atomic Memory fetch-and-operate Routines . . . . . . . . . . . . . . . . . 42

8.13Atomic Memory Operation Routines . . . . . . . . . . . . . . . . . . . . . . . 49

$\begin{array}{ll}\text { 1.0 FINAL } & 3\end{array}$ 
8.14 Point-to-Point Synchronization Routines . . . . . . . . . . . . . . . . . 51

8.15 Barrier Synchronization Routines . . . . . . . . . . . . . . . . . . . . 54

8.16 Reduction Routines . . . . . . . . . . . . . . . . . . . . . . . . . 59

8.17 Collect Routines . . . . . . . . . . . . . . . . . . . . . 83

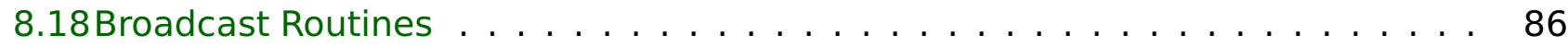

8.19 Lock Routines . . . . . . . . . . . . . . . . . . . . . . . . . . . 90

9 Library Constants . . . . . . . . . . . . . . . . . . . . . . . . . . . 94

9.1 Constants Related To Reduction Operations . . . . . . . . . . . . . . . . . . 94

10 Writing OpenSHMEM Programs . . . . . . . . . . . . . . . . . . 95

10.1 Incorporating OpenSHMEM into Programs . . . . . . . . . . . . . . 95

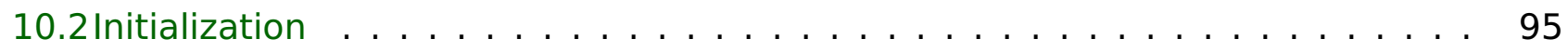

A Deprecated Items . . . . . . . . . . . . . . . . . . . . 96

A.1 Cache Management Routines . . . . . . . . . . . . . . . . . . 96

B Environment Variables . . . . . . . . . . . . . . . . . . . . . . 101

C Compiling and Running Applications . . . . . . . . . . . . . . . . . . . 102

C.1 Compilation. . . . . . . . . . . . . . . . . . . . . . . . . . . . 102

C.2 Applications written in $\mathrm{C}++\ldots \ldots \ldots \ldots \ldots \ldots . \ldots \ldots 2$

C.3 Applications written in Fortran . . . . . . . . . . . . . . . . . 102

D Running Applications . . . . . . . . . . . . . . . . . . . . . . . 103

E Examples . . . . . . . . . . . . . . . . . . . . . . . . . . . . 104

E.1 C Language Examples . . . . . . . . . . . . . . . . . . . . . . . 105

E.2 Fortran Language Examples . . . . . . . . . . . . . . . . . . . . . . 122

F Glossary. . . . . . . . . . . . . . . . . . . . . . 123

F.1 OpenSHMEM Concepts . . . . . . . . . . . . . . . . . . . . . 123

F.2 Data Terminology . . . . . . . . . . . . . . . . . . . . . . . . . 124

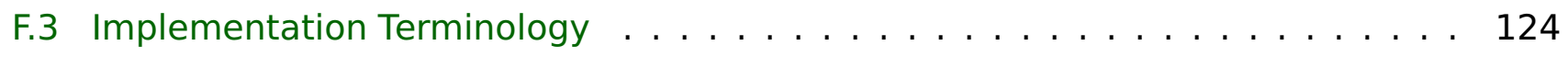

Bibliography . . . . . . . . . . . . . . . . . . . . 126

$\begin{array}{ll}1.0 \text { FINAL } & 4\end{array}$ 


\section{Introduction}

This document defines the elements of the OpenSHMEM Application Programming Interface ${ }^{1}$. The purpose of the OpenSHMEM API is to provide programmers with a standard interface for writing parallel programs using $\mathrm{C}, \mathrm{C}++$ and Fortran with one-sided communication.

More information about the OpenSHMEM project can be found at:

http://www. openshmem.org/

${ }^{1}$ SHMEM and OpenSHMEM are trademarks of Silicon Graphics International Corp. 


\section{What is SHMEM ?}

This section is an introduction to previous work on SHMEM. We begin with a quick overview of the Partitioned Global Address Space model, which is the basis for SHMEM's data sharing strategy.

\subsection{Partitioned Global Address Space}

Conventional Parallel Programming Models can be broadly classified into 2 types:

Shared-Memory Model: in this model all processors interact with a globally available memory space.

Distributed-Memory Model: in this model each processor has its own memory to work with and can only directly access the data that resides in its memory. When a processor needs data from another processor an explicit function call must be made to communicate with the target processor.

The current high performance computing architectures prefer a combination of the above mentioned memory models, which is referred to Partitioned Global Address Space or PGAS for short. In PGAS, each processing element (PE) has access to its own private local memory and also to a shared memory space. This programming model enhances performance by exposing data/thread locality. PGAS programming languages include Unified Parallel C (UPC), Co-Array Fortran (CAF), Titanium, X-10 and Chapel.

More information about PGAS can be found at the PGAS Forum website.[4]

\subsection{SHMEM}

SHMEM is a library API that allows its participating processes (the places where work occurs are called Processing Elements or PEs) to view a Partitioned Global Address Space. Each PE is able to see variables with a common name, but each PE has its own local copy of the variable.

The SHMEM library provides inter-processor communication using data passing and onesided communication techniques. SHMEM differs from the Message Passing Interface (MPI), currently the most widely used communication model, in that the latter generally uses twosided communication (MPI now also includes one-sided calls). In two-sided communication, both sides of the exchange (source and destination) are required to participate actively. The one-sided communication mechanism decouples data transfer and synchronization, reducing communication overhead, resulting in faster communication patterns. Figure 1 shows diagrams for one-sided and two-sided communications. 

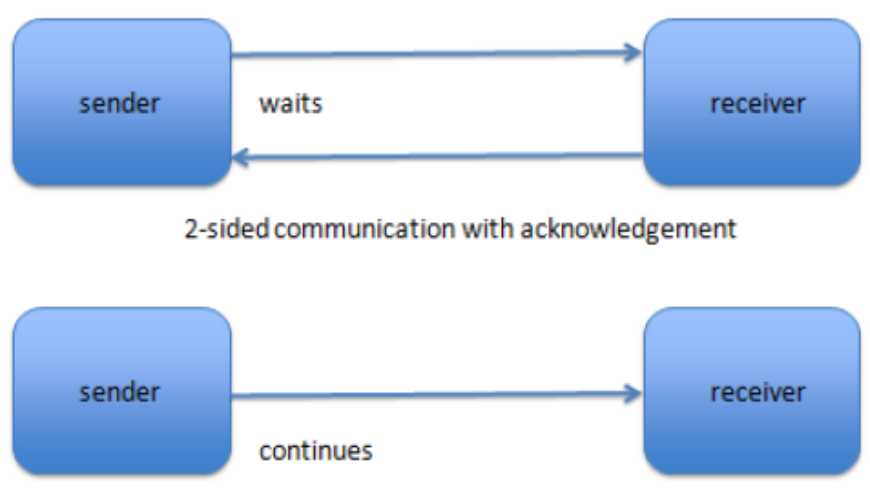

1-sided communication without acknowledgement

Fig. 1: Communication Scheme

The following are some of the communication operations available in SHMEM:

\section{Data Transfers}

(a) One-sided puts : the initiator PE (active side) specifies the local data to be written to the target PE's (passive side) memory.

(b) One-sided gets : an explicit fetch operation is used to copy a variable amount of data from a remote process and store it locally.

Note: By avoiding the need for matching send and receive calls, SHMEM simplifies the communication process by reducing the number of calls required to have one PE interact with other PEs.

\section{Synchronization Mechanisms}

(a) Fence: Ensures ordering of PUT operations to a specific PE.

(b) Quiet: Ensures ordering of PUT operations to all PEs.

(c) Barrier: A collective synchronization routine in which no PE may leave the barrier prior to all PEs entering the barrier.

\section{Collective Communication}

(a) Broadcast: Copy a block of data from one PE to one or more target PEs. 
(b) Collection: Concatenate elements from the source array to a target array over the specified PEs.

(c) Reduction: Perform an associative binary operation over the specified PEs.

\section{Address Manipulation}

(a) Allocating and deallocating memory blocks in the symmetric space.

\section{Locks}

(a) Implementation of mutual exclusion.

\section{Atomic Memory Operations}

(a) Swap, Conditional Swap, Add and Increment

\section{Data Cache control}

(a) Implementation of mechanisms to exploit the capabilities of hardware cache if available.

Note: More information about OpenSHMEM routines can be found in the Library Routines section.

\subsection{History of SHMEM}

Cray SHMEM (MP-SHMEM, LC-SHMEM): Cray first introduced SHMEM in 1993 for its Cray T3D systems. Cray SHMEM was also used in other models: T3E, PVP and XT series.

SGI SHMEM (SGI-SHMEM): Cray Research merged with Silicon Graphics (SGI) in February 1996. At this point SHMEM was incorporated into SGI's Message Passing Toolkit (MPT). The platforms supported were - SGI Irix, Origin and Altix.

Quadrics SHMEM (Q-SHMEM): an optimized API for the Quadrics QsNet interconnect. It included SGI extensions and provided non-blocking puts and gets. A joint effort from HCS Lab \& Quadrics incorporated a program profiling interface called PSHMEM that can aid in the execution analysis of SHMEM programs.

The success of SHMEM's performance attracted several vendors to provide implementations (with varying names and features) for their systems. Some of them include:

HP SHMEM: Based on the Quadrics API. It is included in the UPC product kit. 
Cyclops-64 SHMEM (C64-SHMEM): this SHMEM API supports the Cyclops-64 architecture. Most of the core features of Cray SHMEM are available with some additional interfaces specific to the Cyclops-64 architecture.

IBM SHMEM: An implementation created by IBM intended for internal use only.

TurboSHMEM: This implementation uses IBM's Low-Level API (LAPI) technology to obtain optimized one-sided communication for the put/get operations. This allows applications written with the SHMEM API to run on IBM platforms with minimal source code changes.

GPSHMEM: This implementation of SHMEM aims at providing full portability of applications. It is built mostly with Cray T3D components and functionalities and provides MPI and ARMCI support. This project is no longer maintained. 


\section{The OpenSHMEM Project}

\subsection{What is OpenSHMEM ?}

There are currently various SHMEM implementations available for different platforms. These versions have subtle differences from one another, and generally, code written using any one of these implementations is not directly portable to the others.

OpenSHMEM aims to address this situation by creating a process that builds a new, open specification to consolidate the various extant SHMEM versions into a widely accepted standard.

A result of this process will be an initial reference implementation based on the SGI SHMEM that can serve as a starting point for vendors and library developers. New features and enhancements can be incorporated by the community as agreed and desired. Additionally, OpenSHMEM aims to produce a portable specification enabling programmers to write SHMEM code that will run with little effort on as many different platforms as possible. 


\section{Language Bindings and Conformance}

OpenSHMEM is available with $\mathrm{C}, \mathrm{C}++$ and Fortran bindings. The $\mathrm{C}++$ interface is currently the same as that for $\mathrm{C}$.

An OpenSHMEM implementation can be conformant to one or both of the interfaces. An implementation that provides e.g. only a C interface may claim to conform to the OpenSHMEM specification with respect to the $C$ language, but not to Fortran and should make this clear in its documentation.

An implementation that provides both $\mathrm{C}$ and Fortran bindings may claim complete conformance. 


\section{Memory Model}

The OpenSHMEM specification defines how data is stored in the memory of each PE and how data objects are made remotely accessible to all other PEs.

Data objects can be stored in a private local memory address or in a remotely accessible memory address space. Objects in the private address space can only be accessed by the PE itself; these data objects cannot be accessed by other PEs via OpenSHMEM routines. Remotely accessible objects, however, can be accessed by remote PEs using OpenSHMEM routines. Remotely accessible data objects are also known as Symmetric Objects. An object is symmetric if it has a corresponding object with the same type, size and offset on all other PEs. Examples of Symmetric Objects are static and global variables in $\mathrm{C}$ and $\mathrm{C}++$, which are often allocated at the same address on all PEs where the program is being executed (e.g. in the ELF executable format). See Figure 2 for an example of how Symmetric Memory Objects may be arranged in memory.

OpenSHMEM routines allow the creation of dynamically allocated Symmetric data objects. These objects are created in a special memory region called the Symmetric Heap, which is created during execution at locations determined by the implementation, meaning the Symmetric Heap may be in different memory regions on different PEs. OpenSHMEM has nothing to say regarding the underlying memory layout; it is up to the implementation to decide how to handle the Symmetric Heap.

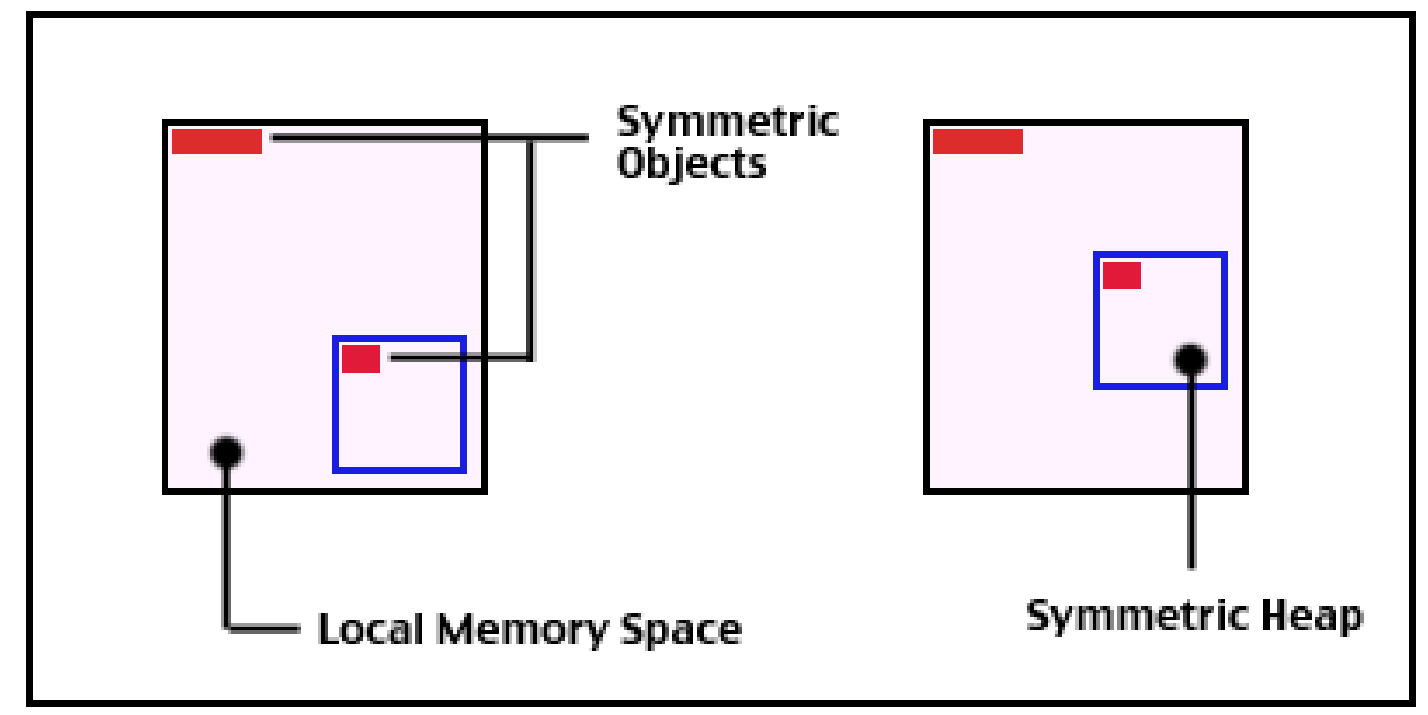

Fig. 2: Example of Symmetric Objects 


\section{Execution Model}

This section describes the Execution Model of an OpenSHMEM application.

OpenSHMEM uses a Single Process Multiple Data (SPMD) approach to express parallelism. An OpenSHMEM application makes use of multiple processors, referred to as Processing Elements or PEs, to complete operations in parallel.

OpenSHMEM requires initialization before using any of the library routines. To this end, the program issues a call to the start_pes() routine. start_pes() performs any required initialization steps, such as setting up the symmetric heap for every PE and creating the PE numbers. The symmetric heap is one of the memory spaces that is remotely accessible by all PEs. The symmetric heap is discussed further in the Memory Model section. The PE numbers are the identifiers used to refer to each of the PEs involved in the execution. These $\mathrm{PE}$ numbers are integers assigned in a monotonically increasing manner from zero to the total number of PEs minus 1.

Data transfer in OpenSHMEM is possible through several one-sided put (for write) and get (for read) operations, as well as various collective routines such as broadcasts and reductions.

Query routines are available to gather information about the execution. OpenSHMEM also provides synchronization routines to coordinate data transfers and other operations.

It is up to the implementation how to handle the finalization of the OpenSHMEM library and any other resources initialized by the library: there is currently no explicit call for the programmer. 


\subsection{Communication Progress}

The OpenSHMEM model assumes that computation and communication are naturally overlapped. OpenSHMEM programs are expected to exhibit progression of communication both with and without OpenSHMEM calls.

Consider a PE that is engaged in a long computation with no OpenSHMEM calls. Other PEs must be able to communicate (put/get, collective, atomic) with that computationally-bound PE without that PE issuing any explicit OpenSHMEM calls.

OpenSHMEM communication calls involving that PE must progress regardless of when that PE next engages in an OpenSHMEM call.

Note to implementers: progress will often be ensured through the use of a dedicated progress thread in software, or through network hardware that offloads communication handling from processors.

\subsection{Atomicity Guarantees}

OpenSHMEM contains a number of routines that operate on symmetric data atomically. These routines guarantee that accesses by OpenSHMEM 's atomic operations will be exclusive, but do not guarantee exclusivity in combination with other routines, either inside OpenSHMEM 's or outside.

For example: during the execution of a remote integer increment operation on a symmetric variable " $x$ ", no other OpenSHMEM atomic operation may access " $x$ ". After the increment, " $x$ " will have increased its value by 1 on the target $P E$, at which point other atomic operations may then modify that " $x$ ". 


\section{Undefined Behavior}

\subsection{Undefined Behavior in OpenSHMEM}

The specification provides guidelines to the expected behavior of various library routines. In cases where routines are improperly used or the input is not in accordance with the specification, undefined behavior may be observed. Depending on the implementation there are many interpretations of undefined behavior.

\begin{tabular}{|l|l|}
\hline Inappropriate Usage & Undefined Behavior \\
\hline \hline Uninitialized library & $\begin{array}{l}\text { If OpenSHMEM is not initialized through a call to } \\
\text { start_pes(), subsequent accesses to OpenSHMEM } \\
\text { routines have undefined results. An implementation } \\
\text { may choose, for example, to try to continue or abort } \\
\text { immediately upon the first call to an uninitialized } \\
\text { routine. }\end{array}$ \\
\hline Accessing non-existent PEs & $\begin{array}{l}\text { If a communications routine accesses a non-existent } \\
\text { PE then the OpenSHMEM library can choose to handle } \\
\text { this situation in an implementation-defined way. For } \\
\text { example, the library may issue an error message } \\
\text { saying that the PE accessed is outside the range of } \\
\text { accessible PEs, or may exit without a warning. }\end{array}$ \\
\hline $\begin{array}{l}\text { Use of non-symmetric } \\
\text { variables }\end{array}$ & $\begin{array}{l}\text { Some routines require remotely accessible variables } \\
\text { to perform their function. A “put" to a non-symmetric } \\
\text { variable can be trapped where possible and the library } \\
\text { can abort the program. Another implementation may } \\
\text { choose to continue either with a warning or silently. }\end{array}$ \\
\hline Non-symmetric Variables & $\begin{array}{l}\text { The symmetric memory management routines are } \\
\text { collectives, which means that all PEs in the program } \\
\text { must issue the same shmalloc() call with the same } \\
\text { size request. OpenSHMEM implementations should } \\
\text { detect the size mismatch and return error information } \\
\text { to the caller. Implementations may also produce an } \\
\text { error message. Program behavior after a mismatched } \\
\text { shmalloc() call is undefined. }\end{array}$ \\
\hline
\end{tabular}




\section{Library Routines}

\subsection{Initialization Routines}

\subsection{1 start_pes}

\section{Summary}

Initializes OpenSHMEM.

\section{Synopsis}

$\mathrm{C} / \mathrm{C}++$ :

void start_pes (int npes);

Fortran:

INTEGER npes

CALL START_PES (npes)

\section{Parameters}

npes Unused. Should be set to 0 .

\section{Constraints}

- If start_pes() is called multiple times, subsequent calls have no effect.

- An OpenSHMEM application must make a call to start_pes() before being able to call any other OpenSHMEM routine. Calling another OpenSHMEM library routine before calling start_pes() results in undefined behavior.

\section{Effect}

Initializes the execution environment of the PE. This routine is responsible inter alia for setting up the symmetric heap on the calling PE, and the creation of the PE numbers. Upon successful return from this routine, the calling PE will be able to communicate with and transfer data to other PEs. 


\section{Return Values}

None

\subsection{Query Routines}

The OpenSHMEM query routines provide information about the program execution.

\subsection{1 num_pes, shmem_n_pes}

\section{Summary}

Returns the number of processing elements (PEs) used to run the application.

\section{Synopsis}

$\mathrm{C} / \mathrm{C}++$ :

int_num_pes (void) ;

int shmem_n_pes (void);

Fortran:

INTEGER I

$I=$ NUM_PES ( )

$\mathbf{I}=$ SHMEM_N_PES ()

\section{Parameters}

None.

\section{Constraints}

Note that the Fortran name is num_pes whereas the $\mathrm{C} / \mathrm{C}++$ name has a leading underscore, _num_pes.

\section{Effect}

num_pes() or shmem_n_pes() returns the total number of PEs running in an application. 


\section{Return Values}

The total number of PEs running the application. PEs are numbered $0 . .(n-1)$.

\subsection{2 my_pe, shmem_my_pe}

\section{Summary}

Returns the PE number of the calling PE.

\section{Synopsis}

$\mathrm{C} / \mathrm{C}++$ :

int_my_pe (void);

int shmem_my_pe (void);

Fortran:

INTEGER I

$\mathbf{I}=\mathbf{M Y} \_\mathbf{P E}()$

$\mathbf{I}=$ shmem_MY $\_\mathbf{P E}()$

\section{Parameters}

None.

\section{Constraints}

Note that the Fortran name is $\mathbf{m y}$ _pe whereas the $\mathrm{C} / \mathrm{C}++$ name has a leading underscore, _my_pe.

\section{Effect}

my_pe() or shmem_my_pe() returns the PE number of the calling PE. PEs are numbered from $0 . .(n-1)$.

\section{Return Values}

The PE number of the calling PE. 


\subsection{Accessibility Query Routines}

\subsection{1 shmem_pe_accessible}

\section{Summary}

This routine determines if a target PE is reachable from the calling PE.

\section{Synopsis}

$\mathrm{C} / \mathrm{C}++$ :

int shmem_pe_accessible (int pe);

Fortran:

LOGICAL LOG，SHMEM_PE_ACCESSIBLE

INTEGER pe

LOG $=$ SHMEM_PE_ACCESSIBLE $($ pe $)$

\section{Parameters}

pe PE number of the target PE.

\section{Constraints}

- pe must be a PE number. For more information about how PE numbers are assigned please refer to the Execution Model section.

\section{Effect}

This routine returns a value that indicates whether the calling PE is able to perform OpenSHMEM communication operations with the target PE.

\section{Return Values}

In $\mathrm{C} / \mathrm{C}++$, shmem_pe_accessible() returns 1 if the specified PE is a valid target PE for OpenSHMEM functions; otherwise, it returns 0 . In Fortran, shmem_pe_accessible() returns .TRUE. if the specified PE is a valid target PE for OpenSHMEM functions; otherwise, it returns .FALSE.. 


\subsection{2 shmem_addr_accessible}

\section{Summary}

This routine indicates if an address is accessible via OpenSHMEM operations from the specified target PE.

\section{Synopsis}

$\mathrm{C} / \mathrm{C}++$ :

int shmem_addr_accessible (void *addr, int pe);

Fortran:

LOGICAL LOG, SHMEM_ADDR_ACCESSIBLE

INTEGER pe

LOG $=$ SHMEM_ADDR_ACCESSIBLE (addr, pe $)$

\section{Parameters}

addr The address to the memory block in the local symmetric heap.

pe PE number of the target PE.

\section{Constraints}

- pe must be a PE number. For more information about how PE numbers are assigned please refer to the Execution Model section.

\section{Effect}

shmem_addr_accessible() determines if the target PE pe is accessible via OpenSHMEM communication routines and that the address addr is in a symmetric segment with respect to the target PE pe.

\section{Return Values}

In C/C++, shmem_addr_accessible() returns 1 if the data object at address addr is symmetric and can be accessed via OpenSHMEM functions; otherwise, it returns 0 . In Fortran, shmem_addr_accessible() .TRUE. if the data object at address addr is symmetric and can be accessed via OpenSHMEM functions; otherwise, it returns .FALSE.. 


\subsection{Symmetric Heap Routines}

The OpenSHMEM Symmetric Heap routines manage memory blocks inside the symmetric heap. The total size of the symmetric heap is determined at program start.

\subsection{1 shmalloc}

\section{Summary}

This routine allocates a block of memory in the symmetric heap of the calling PE.

\section{Synopsis}

$\mathrm{C} / \mathrm{C}++\mathrm{:}$

void *shmalloc (size_t size);

\section{Parameters}

size Size of the requested memory block, in bytes.

\section{Constraints}

- All PEs must call this routine at the same point of the execution path; otherwise, undefined behavior results.

- All PEs must call this routine with the same size value; otherwise, undefined behavior results.

- The parameter size must be less than or equal to the amount of symmetric heap space available for the calling PE; otherwise shmalloc returns NULL.

\section{Effect}

The shmalloc() routine allocates a block of memory of at least size bytes from the symmetric heap of the calling PE, and returns a pointer to the allocated block.

shmalloc() calls shmem_barrier_all() before returning to ensure that all the PEs participate. This guarantees symmetric allocation, and that the memory on all the PEs is available to the other PEs. 


\section{Return Values}

The shmalloc() routine returns a pointer to the allocated block; otherwise a null pointer is returned if no block could be allocated.

\subsection{2 shmemalign}

\section{Summary}

This routine allocates a block from the symmetric heap with a byte alignment specified by the programmer.

\section{Synopsis}

$\mathrm{C} / \mathrm{C}++$ :

void *shmemalign (size_t alignment, size_t size);

\section{Parameters}

alignment Size of the alignment block, in bytes.

size Size for the memory block, in bytes.

\section{Constraints}

- Values for parameters alignment and size must be positive integer values.

- alignment is a power of 2 ; power $\geq 3$.

- The parameter size must be less than or equal to the amount of symmetric heap space available for the calling PE; otherwise shmemalign returns NULL.

\section{Effect}

The shmemalign() routine allocates a memory block of size bytes in the symmetric heap, with an alignment of alignment bytes.

shmemalign() calls shmem_barrier_all() before returning to ensure that all the PEs participate. This guarantees symmetric allocation, and that the target memory on all the PEs is available to the other PEs. 


\section{Return Values}

This routine returns a pointer to the allocated block of memory; otherwise a null pointer is returned.

\subsection{3 shrealloc}

\section{Summary}

This routine expands or reduces the size of the block to which ptr points, depending on the provided size parameter.

\section{Synopsis}

$\mathrm{C} / \mathrm{C}++$ :

void $\star$ shrealloc (void $\star$ ptr, size_t size);

\section{Parameters}

ptr Pointer to a memory block previously allocated with shmalloc() or shrealloc() to be reallocated.

size New size for the memory block, in bytes.

\section{Constraints}

- All PEs must call this routine at the same point of the execution path; otherwise, undefined behavior results.

- All PEs must call this routine with the same parameters; otherwise different symmetric heap addresses may be returned to each PE.

- The parameter size must be an integer greater than or equal to zero.

- The parameter size must be lesser than or equal to the amount of symmetric heap space available for the calling PE; otherwise shrealloc returns NULL. 


\section{Effect}

The shrealloc() routine changes the size of the memory block to which ptr points to, and returns a pointer to the memory block. The contents of the memory block are preserved up to the lesser of the new and old sizes. If the new size is larger, the value of the newly allocated portion of the block is indeterminate. In case shrealloc() is unable to extend the size of the memory block at its current location, the routine may move the block elsewhere in the symmetric heap while ensuring that contents of the block are preserved. In case of relocation, shrealloc() will return a pointer to the new location.

If ptr is null, shrealloc() behaves exactly like shmalloc(). If size is 0 and ptr is not null, the memory block is deallocated. Otherwise, if ptr does not match a pointer earlier returned by a symmetric heap function, or if the space has already been deallocated, shrealloc() will return a null pointer. If the space cannot be allocated, the block to which ptr points to is unchanged.

shrealloc() calls shmem_barrier_all() before returning to ensure that all the PEs participate. This guarantees symmetric allocation, and that the target memory on all the PEs is available to the other PEs.

\subsection{4 shfree}

\section{Summary}

Frees a memory block previously allocated in the symmetric heap.

\section{Synopsis}

$\mathrm{C} / \mathrm{C}++$ :

void shfree (void $\star$ ptr);

\section{Parameters}

ptr Pointer to a memory block previously allocated with shmalloc() or shrealloc() to be deallocated.

\section{Constraints}

None. 


\section{Effect}

This routine causes the block to which ptr points to, to be deallocated; that is, made available for further allocation.

If ptr is a null pointer, no action occurs; otherwise, if the argument does not match a pointer earlier returned by a symmetric heap function, or if the space has already been deallocated, shfree() returns.

shfree() calls shmem_barrier_all() before returning to ensure that all the PEs participate. This guarantees symmetric deallocation within the heap.

\section{Return Values}

None.

\subsubsection{SHPALLOC}

\section{Summary}

This routine allocates a block of memory in the symmetric heap of the calling PE.

\section{Synopsis}

Fortran:

POINTER (addr, $\mathbf{A}(1)$ )

INTEGER (length, errcode, abort)

CALL SHPALLOC (addr, length, errcode, abort)

\section{Parameters}

addr First word address of the allocated block (output variable).

length Number of words of memory requested (input variable). One word is 32 bits.

errcode Error code is 0 if no error was detected; otherwise, it is a negative integer code for the type of error (output variable).

abort Abort code; nonzero requests abort on error; 0 requests an error code (input variable). 


\section{Constraints}

- All PEs must call this routine at the same point of the execution path; otherwise, undefined behavior results.

- All PEs must call this routine with the same parameters; otherwise different symmetric heap addresses may be returned to each PE..

- length must be lesser than or equal to the amount of symmetric heap space available for the calling PE.

\section{Effect}

The SHPALLOC() routine allocates a block of memory in the symmetric heap of the calling $\mathrm{PE}$, and returns the address of the allocated block via the addr parameter.

shpalloc() calls shmem_barrier_all() before returning to ensure that all the PEs participate. This guarantees symmetric allocation, and that the target memory on all the PEs is available to the other PEs.

\section{Return Values}

The SHPALLOC() routine returns the address of the allocated memory block via the addr parameter and a result code via the errcode parameter. The possible values for errcode are:

0 Operation was successful .

-1 Length is not an integer greater than 0 .

-2 No more memory is available from the system (checked if the request cannot be satisfied from the available blocks on the symmetric heap).

\subsubsection{SHPDEALLC}

\section{Summary}

Frees a memory block previously allocated in the symmetric heap. 


\section{Synopsis}

Fortran:

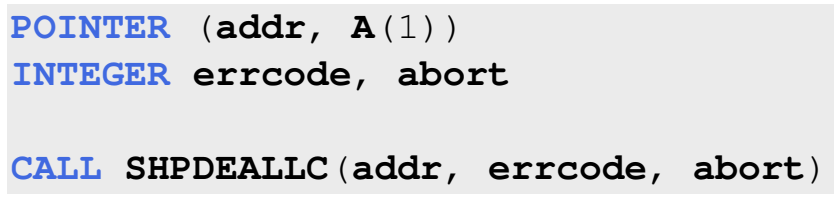

\section{Parameters}

addr First word address of the block to deallocate (input).

errcode Error code is 0 if no error was detected; otherwise, it is a negative integer code for the type of error (output).

abort Abort code. Nonzero requests abort on error; 0 requests an error code (input).

\section{Constraints}

- All PEs must call this routine at the same point of the execution path; otherwise, undefined behavior results.

- All PEs must call this routine with the same parameters; otherwise different symmetric heap addresses may be returned to each PE.

- The parameter addr must be the address of a block of memory allocated in the symmetric heap.

- To maintain symmetric heap consistency, all processing elements (PEs) in a program must call SHPDEALLC() with the same value of addr; if any PEs fail to call this routine, it may result in undefined behavior.

\section{Effect}

This routine causes the block at address addr, to be deallocated, that is, made available for further allocation. If addr is not an address in the symmetric heap, an error code is returned via the parameter errcode.

shdeallc() calls shmem_barrier_all() before returning to ensure that all the PEs participate. This guarantees symmetric deallocation. 


\section{Return Values}

This routine returns a result code via the errcode parameter. Possible values for errcode are:

0 Operation was successful .

-3 Address is outside the bounds of the symmetric heap.

-4 Block is already free.

-5 Address is not at the beginning of the block.

\subsubsection{SHPCLMOVE}

\section{Summary}

This routine expands or reduces the size of the memory block with address addr, depending on the provided length parameter.

\section{Synopsis}

Fortran:

POINTER (addr, A(1))

INTEGER (length, errcode, abort)

CALL SHPCLMOVE (addr, length, status, abort)

\section{Parameters}

addr On entry, first word address of the block to change; on exit, the new address of the block if it was moved. (input and output)

length Requested new total length in words (input). One word is 32 bits.

status Status code. See Return Values below for possible status codes (output).

abort Abort code. Nonzero requests abort on error; 0 requests an error code (input). 


\section{Constraints}

- All PEs must call this routine at the same point of the execution path; otherwise, undefined behavior results.

- All PEs must call this routine with the same parameters; otherwise different symmetric heap addresses may be returned to each PE.

- The parameter addr must be the address of a block of memory allocated in the symmetric heap.

\section{Effect}

The SHPCLMOVE() routine either extends a symmetric heap block if the block is followed by a large enough free block or copies the contents of the existing block to a larger block and returns a status code indicating that the block was moved. This function also can reduce the size of a block if the new length is less than the old length. The function may move the memory block to a new location, in which case the address of the new location is returned.

shpclmove() calls shmem_barrier_all() before returning to ensure that all the PEs participate. This guarantees symmetric allocation, and that the target memory on all the PEs is available to the other PEs.

\section{Return Values}

The address of the new block passed back via the parameter addr.

Possible codes returned via the status parameter:

0 The memory block was resized at its initial location in the symmetric heap.

1 The memory block was moved to a new location in the symmetric heap.

-1 Length is not an integer greater than 0 .

-2 No more memory is available from the system (checked if the block cannot be extended and the free space list does not include a large enough block).

-3 Address is outside the bounds of the symmetric heap.

-4 Block is already free.

-5 Address is not at the beginning of a block. 


\subsection{Remote Pointer Operations}

\subsection{1 shmem_ptr}

\section{Summary}

Returns a pointer to a data object of a target PE.

\section{Synopsis}

$\mathrm{C} / \mathrm{C}++$ :

void $\star s h m e m$ ptr (void *target, int pe);

Fortran:

POINTER (PTR, POINTEE)

INTEGER pe

PTR = SHMEM_PTR (target, pe)

\section{Parameters}

target Address of the symmetric data object.

pe The PE number of the target PE.

\section{Constraints}

- The shmem_ptr() function only returns a non-NULL value on systems where ordinary memory loads and stores are used to implement OpenSHMEM put and get operations.

- target must be the address of a symmetric data object.

- pe must be a PE number. For more information about how PE numbers are assigned please refer to the Execution Model section. 


\section{Effect}

The shmem_ptr() routine returns an address that can be used to directly reference target on the target PE pe. The programmer must be able to assign this address to a pointer and perform ordinary loads and stores to this target address.

When a sequence of loads (gets) and stores (puts) to a data object on a target PE does not match the access pattern provided in a OpenSHMEM data transfer routine like shmem put32() or shmem_real_iget(), the shmem_ptr() function can provide an efficient means to accomplish the communication.

\section{Return Values}

Returns a pointer to the data object on the target PE or NULL if the target object cannot be accessed directly.

\subsection{Elemental Put Routines}

\subsection{1 shmem_TYPE_p}

Summary These routines provide a low latency mechanism to write basic types (short, int, float, double, long, long long, long double) to symmetric data objects on target PEs.

\section{Synopsis $\mathrm{C} / \mathrm{C}++$ :}

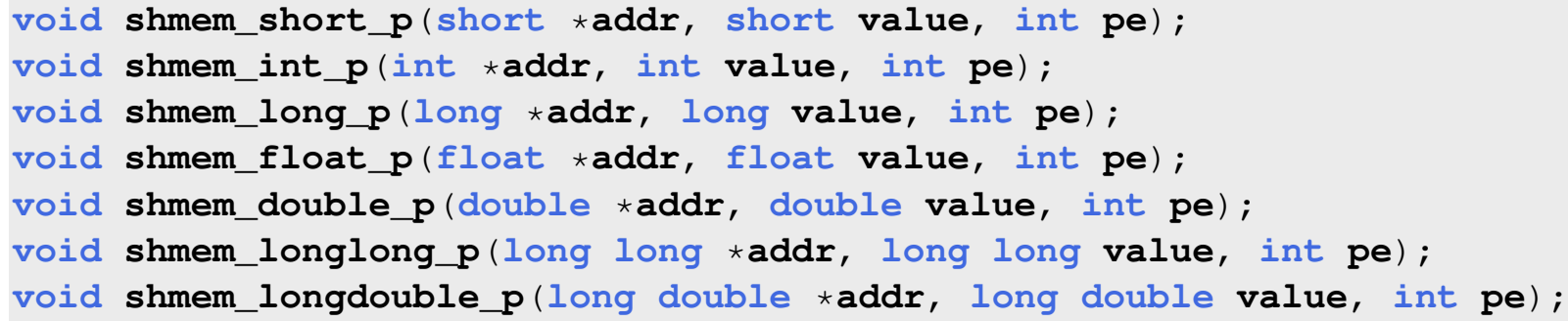

\section{Parameters}

addr Address of the symmetric data object where to save the data on the target pe. value The value to be transferred to addr on the target pe. pe PE number of the target PE. 


\section{Constraints}

- addr must be the address of a symmetric data object.

- pe must be a PE number. For more information about how PE numbers are assigned please refer to the Execution Model section.

Effect The shmem_TYPE_p() routines write value to a symmetric array element or scalar data object of the target PE indicated by the parameter pe. These routines start the target transfer and may return before the data is delivered to the target PE.

\section{Return Values None}

\subsection{Block Data Put Routines}

\subsection{1 shmem_put}

\section{Summary}

These routines copy contiguous data from a local object to an object on the destination PE.

\section{Synopsis}

$\mathrm{C} / \mathrm{C}++$ :

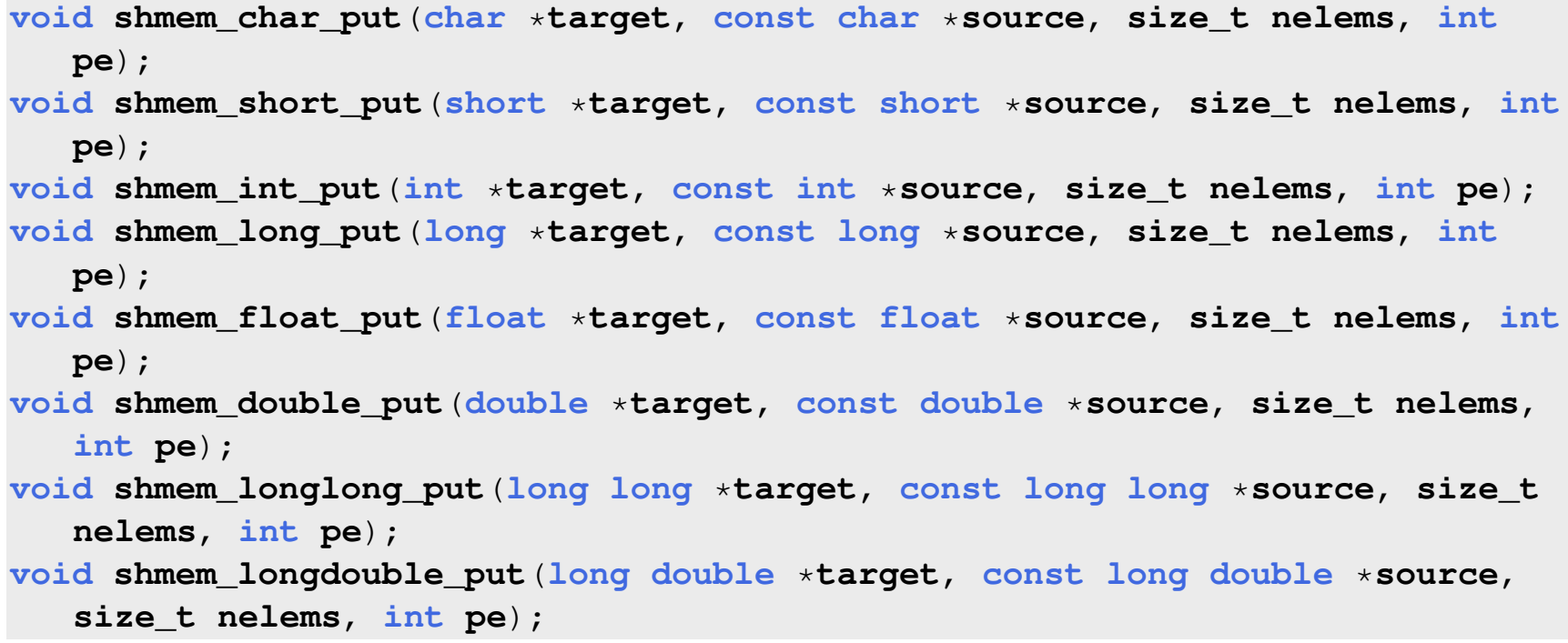




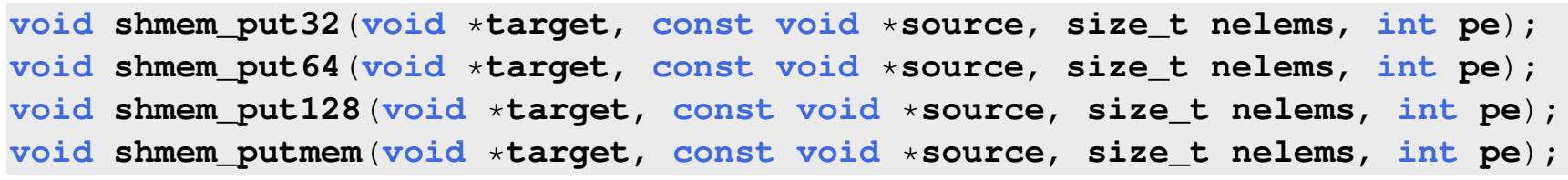

Fortran:

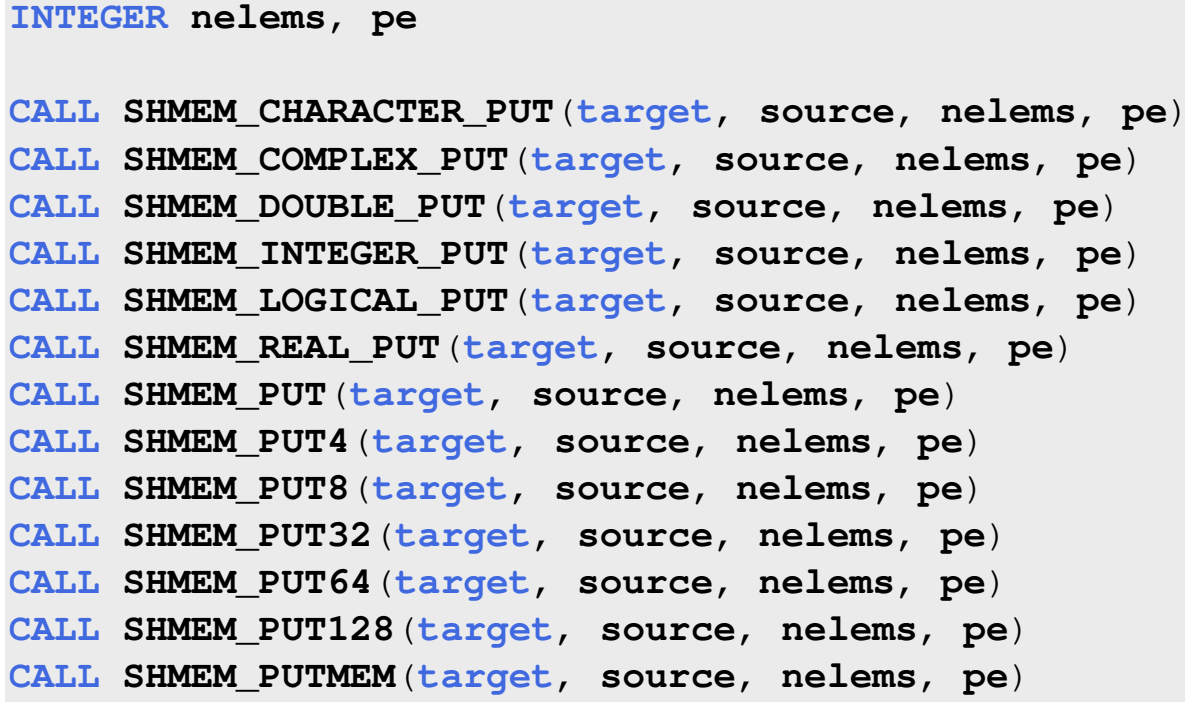

\section{Parameters}

target Address of the symmetric data object where to save the data on the target pe.

source Address of the data to be transferred to the target data object.

nelems The number of elements in the target and source objects.

pe PE number of the target PE.

\section{Constraints}

- target must be the address of a symmetric data object.

- source must have the same type as target.

- nelems must be of type integer. If you are using Fortran, it must be a constant, variable, or array element of default integer type.

- pe must be a PE number. For more information about how PE numbers are assigned please refer to the Execution Model section. 


\section{Effect}

These routines transfer nelems elements of the data object at address source on the calling PE, to the data object at address target on the target PE pe. These routines start the target transfer and return after the data has been copied out of the source array on the local PE. However, this does not guarantee delivery to the target PE. The delivery of data into the data object on the destination PE from different put calls may occur in any order. Because of this, two successive put operations may deliver data out of order unless a call to shmem. fence() is introduced between the two calls.

\section{Return Values}

None

\subsection{Strided Put Routines}

\subsection{1 shmem_iput}

\section{Summary}

These routines copy strided data from the local PE to a strided data object on the destination PE.

\section{Synopsis}

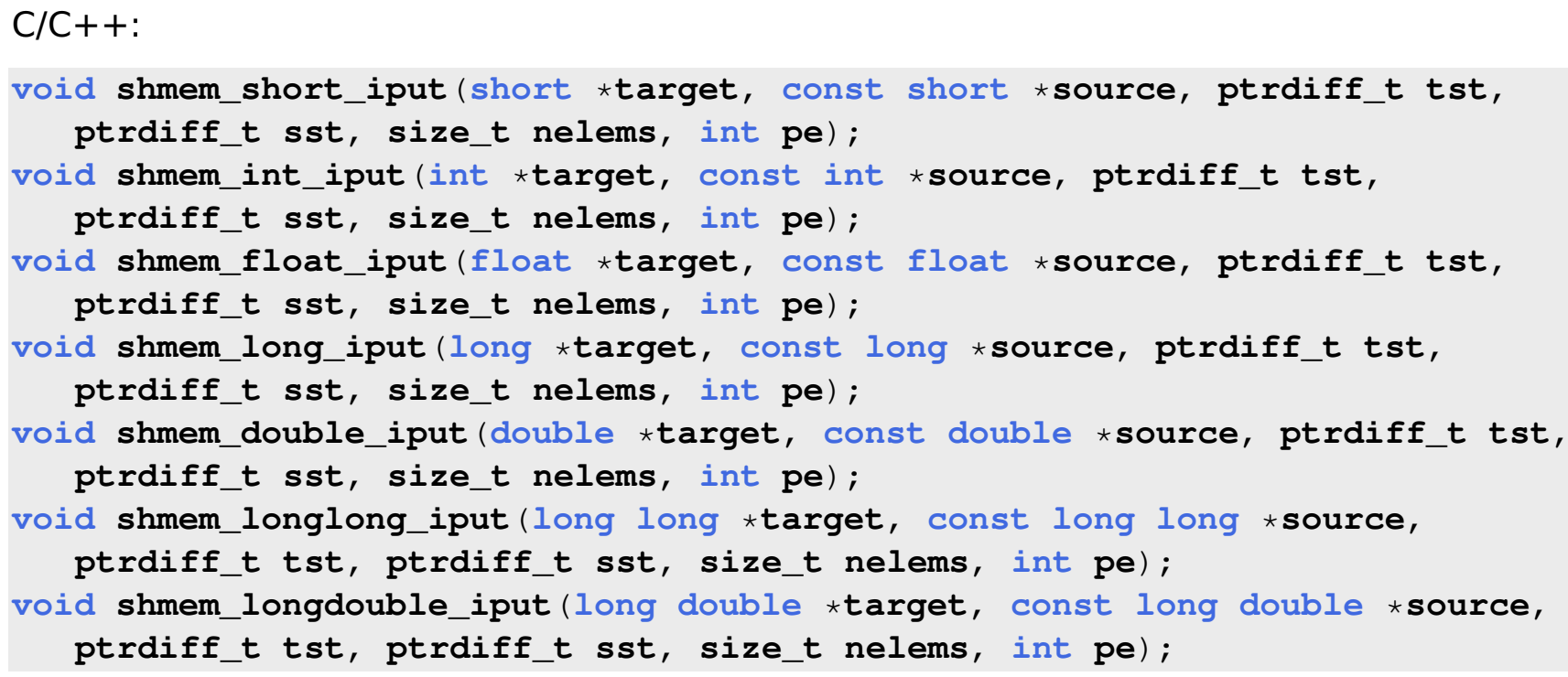




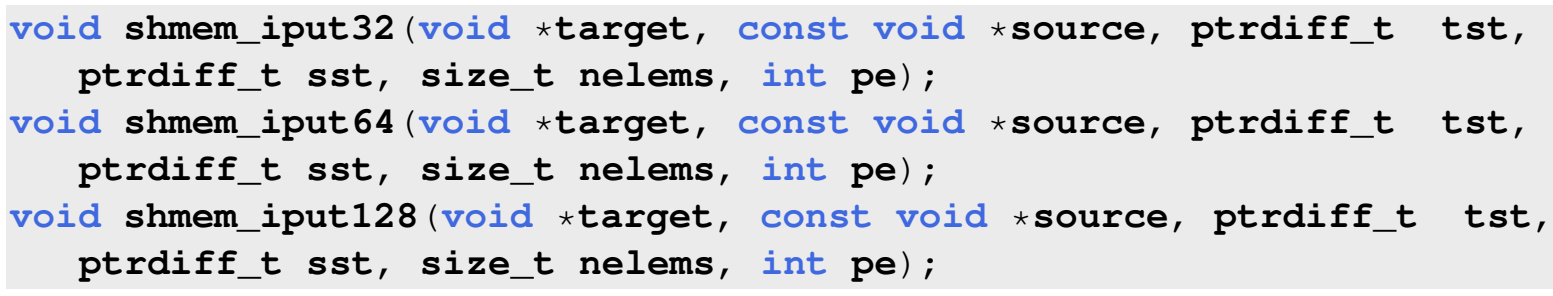

Fortran:

INTEGER tst, sst, nelems, pe

CALI SHMEM_COMPLEX_IPUT (target, source, tst, sst, nelems, pe)

CALI SHMEM_DOUBLE_IPUT (target, source, tst, sst, nelems, pe)

CALI SHMEM_INTEGER_IPUT (target, source, tst, sst, nelems, pe)

CALI SHMEM_LOGICAL_IPUT (target, source, tst, sst, nelems, pe)

CALI SHMEM_REAL_IPUT (target, source, tst, sst, nelems, pe)

CALL SHMEM_IPUT4 (target, source, tst, sst, nelems, pe)

CALI SHMEM_IPUT8 (target, source, tst, sst, nelems, pe)

CALL SHMEM_IPUT32 (target, source, tst, sst, nelems, pe)

CALL SHMEM_IPUT64 (target, source, tst, sst, nelems, pe)

CALI SHMEM_IPUT128 (target, source, tst, sst, nelems, pe)

\section{Parameters}

target Address of the symmetric data object where to save the data on the target pe.

source Address of the data to be transferred to the target data object.

tst The stride between consecutive elements in the target array. tst must be of type integer. If you are using Fortran, it must be a default integer value.

sst The stride between consecutive elements in the source array. sst must be of type integer. If you are using Fortran, it must be a default integer value.

nelems Number of elements in the target and source objects. nelems must be of type integer. If you are using Fortran, it must be a constant, variable, or array element of default integer type.

pe PE number of the target PE.

\section{Constraints}

- target must be the address of a symmetric data object. 
- source must have the same type as target.

- The strides tst and sst are scaled by the element size of the target and source arrays respectably. A value of 1 indicates contiguous data. tst and sst must be of type integer. If you are using Fortran, it must be a default integer value.

- pe must be a PE number. For more information about how PE numbers are assigned please refer to the Execution Model section.

- Depending on the routine being called, source and target must conform to the following typing constraints:

- In shmem_iput32() and shmem_iput4() they can only have a non character type that has a storage size equal to 32 bits.

- In shmem_iput64() and shmem_iput8() they can only have a non character type that has a storage size equal to 64 bits.

- In shmem_iput128() they can only have a non character type that has a storage size equal to 128 bits.

- In shmem_short_iput() they must be of type short.

- In shmem_int_iput() they must be of type int.

- In shmem_float_iput() they must be of type float.

- In shmem_double_iput() they must be of type double.

- In shmem_long_iput() they must be of type long.

- In shmem_longlong_iput() they must be of type long long.

- In shmem_longdouble_iput() they must be of type long double.

- In SHMEM_COMPLEX_IPUT() they must be of type complex of default size.

- In SHMEM_DOUBLE_IPUT() they must be of type double precision.

- In SHMEM_INTEGER_IPUT() they must be of type integer.

- In SHMEM_LOGICAL_IPUT() they must be of type logical.

- In SHMEM_REAL_IPUT() they must be of type real.

\section{Effect}

The shmem_iput() routines read the elements of a local array (source) and write them to a target array (target) on the PE indicated by pe. These routines return when the data has been copied out of the source array on the local PE but not necessarily before the data has been delivered to the target data object. 


\section{Return Values}

None.

\subsection{Elemental Data Get Routines}

\subsection{1 shmem_TYPE_g}

\section{Summary}

These routines provide a low latency mechanism to retrieve basic types (short, int, float, double, long) from symmetric data objects on target PEs.

\section{Synopsis}

$\mathrm{C} / \mathrm{C}++$ :

short shmem_short_g (short $\star$ addr, int pe);

int shmem_int_g(int $\star$ addr, int pe);

long shmem_long_g(long $\star$ addr, int pe);

float shmem_float_g(float *addr, int pe);

double shmem_double_g(double $\star$ addr, int pe) ;

long long shmem_longlong_g(long long $\star$ addr, int pe);

long double shmem_longdouble_g(long double $\star$ addr, int pe);

\section{Parameters}

addr Address of the symmetric data object that contains the data to be read. pe PE number of the target PE.

\section{Constraints}

- addr must be the address of a symmetric data object.

\section{Effect}

Retrieves the value at the symmetric address addr of the target PE pe. 


\section{Return Value}

The value at the symmetric address addr on PE pe.

\subsection{Block Data Get Routines}

\subsection{1 shmem_get}

\section{Summary}

These routines retrieve data from a contiguous data object on a target PE.

\section{Synopsis}

$\mathrm{C} / \mathrm{C}++$ :

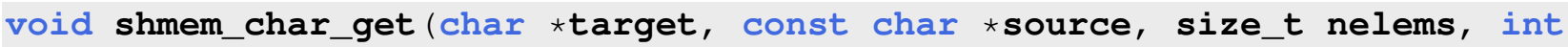
pe) ;

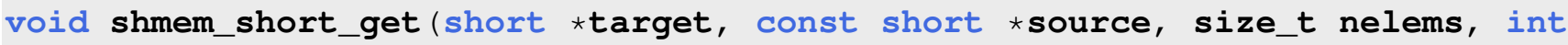
pe) ;

void shmem_int_get (int *target, const int *source, size_t nelems, int pe);

void shmem_long_get(long *target, const long $\star$ source, size_t nelems, int pe) ;

void shmem_float_get (float *target, const float *source, size_t nelems, int pe) ;

void shmem_double_get (double *target, const double source, size_t nelems, int pe);

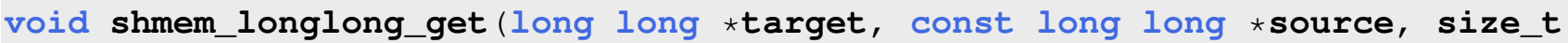
nelems, int pe);

void shmem_longdouble_get(long double *target, const long double $\star s o u r c e$, size_t nelems, int pe);

void shmem_get 32 (void *target, const void *source, size_t nelems, int pe); void shmem_get 64 (void *target, const void *source, size_t nelems, int pe);

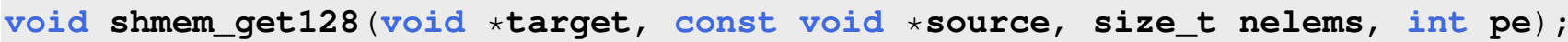
void shmem_getmem(void *target, const void *source, size_t nelems, int pe);

Fortran:

INTEGER nelems, pe

CALL SHMEM_CHARACTER_GET (target, source, nelems, pe)

CALL SHMEM_COMPLEX_GET (target, source, nelems, pe) 


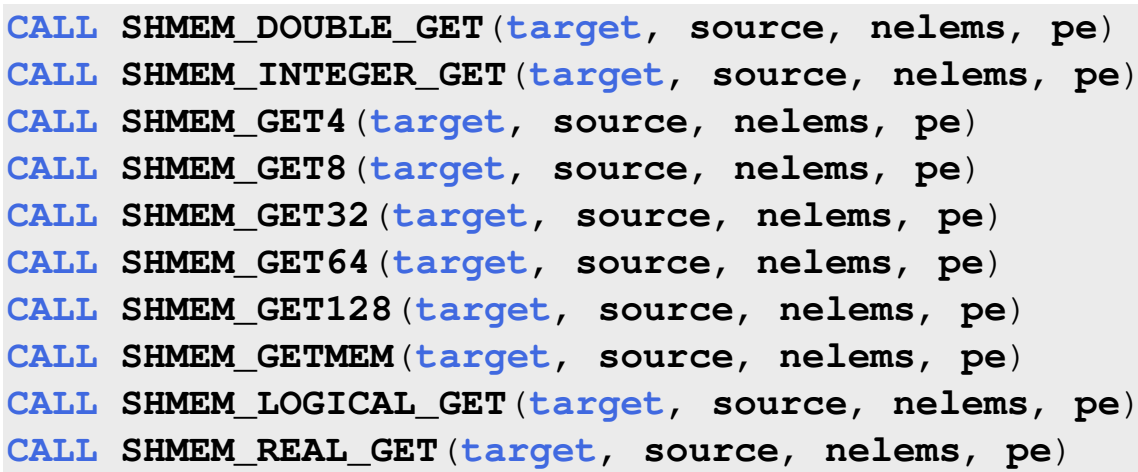

\section{Parameters}

target Address of the local data object in which to save the data.

source Address of the symmetric data object on the target pe with the data to be retrieved. nelems Number of elements in the target and source arrays.

pe Identifier of the target PE.

\section{Constraints}

- source must be the address of a symmetric data object.

- In $\mathrm{C} / \mathrm{C}++$ nelems must be of type integer. If you are using Fortran, it must be a constant, variable, or array element of default integer type.

\section{Effect}

The shmem_get() routines transfer nelems elements of the data object at address source on the target PE (pe), to the data object at address target on the local PE. These routines return after the data has been copied to address target on the local pe.

\section{Return Values}

After successful completion, the retrieved data will be available at address target. 


\subsection{Strided Get Routines}

\subsection{1 shmem_iget}

\section{Summary}

The strided get routines copy strided data located on a target PE to a local strided data object.

\section{Synopsis}

\section{$\mathrm{C} / \mathrm{C}++$ :}

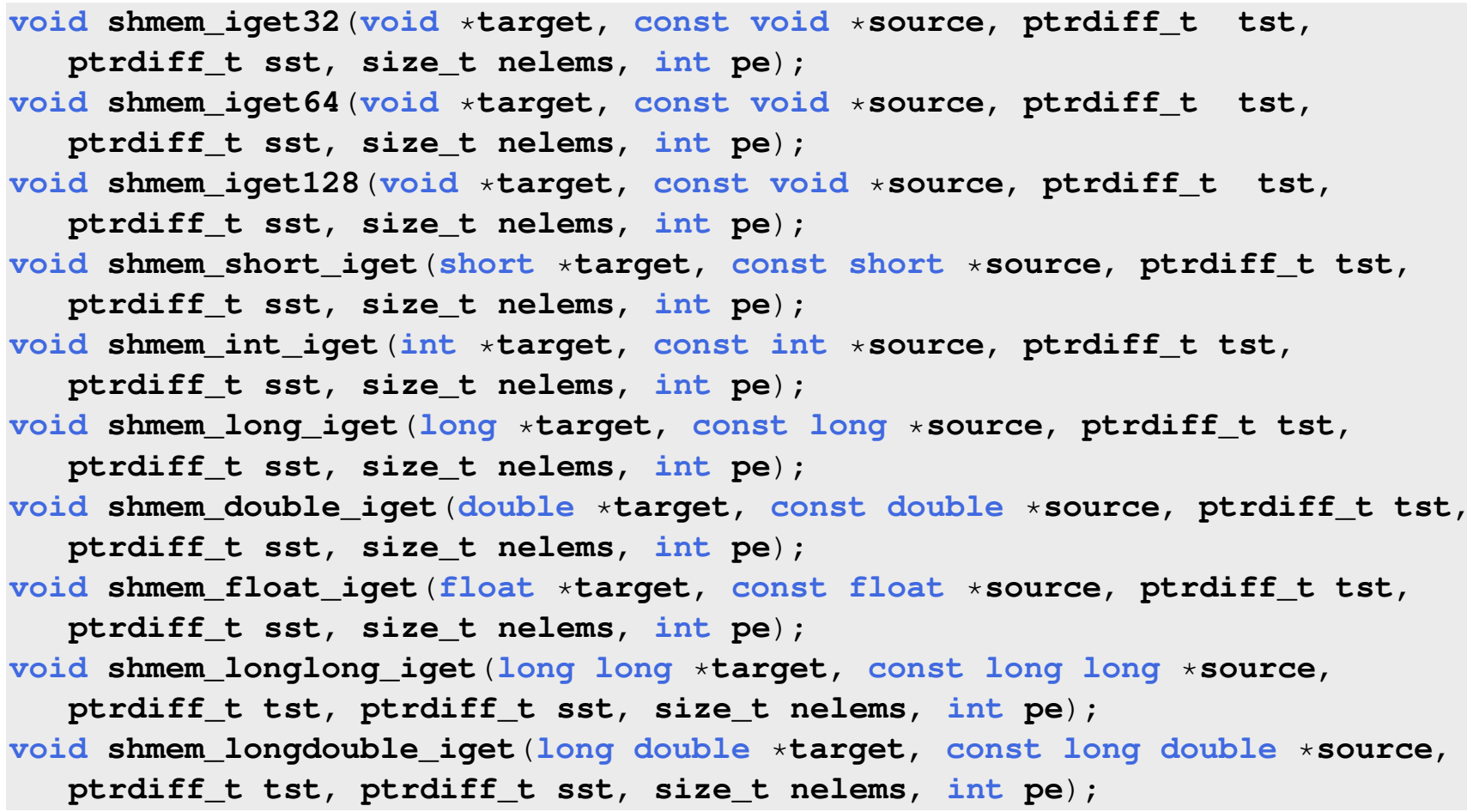

Fortran:

INTEGER tst, sst, nelems, pe

CALL SHMEM_IGET4 (target, source, tst, sst, nelems, pe)

CALL SHMEM_IGET8 (target, source, tst, sst, nelems, pe)

CALL SHMEM_IGET32 (target, source, tst, sst, nelems, pe)

CALL SHMEM_IGET64 (target, source, tst, sst, nelems, pe)

CALL SHMEM_IGET128 (target, source, tst, sst, nelems, pe) 


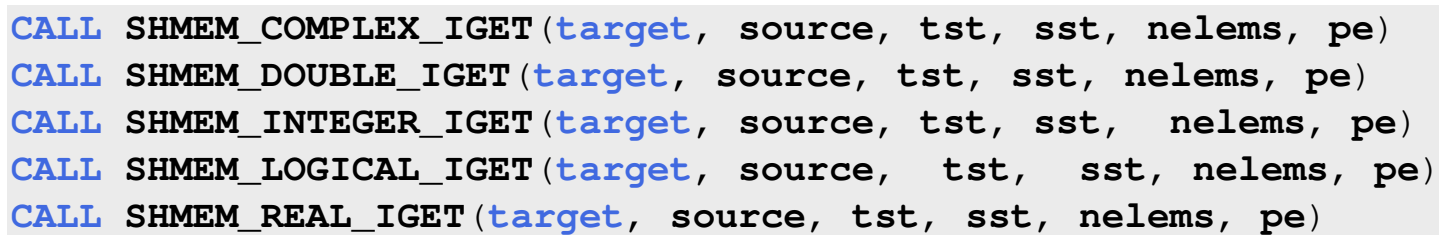

\section{Parameters}

target Address of the data object in which to save the data on the local pe.

source Address of the symmetric data object on the target pe with the data to be retrieved. tst The stride between consecutive elements in the target array.

sst The stride between consecutive elements in the source array.

nelems Number of elements in the target and source objects.

pe PE number of the target PE.

\section{Constraints}

- source must be the address of a symmetric data object.

- source must have the same type as target.

- In $\mathrm{C} / \mathrm{C}++$ tst and sst must be of type integer. If you are using Fortran, they must be a default integer value.

- The strides tst and sst are scaled by the element size of the target and source arrays respectably. A value of 1 indicates contiguous data. tst and sst must be of type integer. If you are using Fortran, it must be a default integer value.

- In $\mathrm{C} / \mathrm{C}++$ nelems must be of type integer. If you are using Fortran, it must be a constant, variable, or array element of default integer type.

- Depending on the routine being called, source and target must conform to the following typing constraints:

- In shmem_iget32() and shmem_iget4() they can only have a non character type that has a storage size equal to 32 bits.

- In shmem_iget64() and shmem_iget8() they can only have a non character type that has a storage size equal to 64 bits. 
- In shmem_iget128() they can only have a non character type that has a storage size equal to 128 bits.

- In shmem_short_iget() they must be of type short.

- In shmem_int_iget() they must be of type int.

- In shmem_float_iget() they must be of type float.

- In shmem_double_iget() they must be of type double.

- In shmem_long_iget() they must be of type long.

- In shmem_longlong_iget() they must be of type long long.

- In shmem_longdouble_iget() they must be of type long double.

- In SHMEM_COMPLEX_IGET() they must be of type complex of default size.

- In SHMEM_DOUBLE_IGET() they must be of type double precision.

- In SHMEM_INTEGER_IGET() they must be of type integer.

- In SHMEM_LOGICAL_IGET() they must be of type logical.

- In SHMEM_REAL_IGET() they must be of type real.

\section{Effect}

The strided get routines retrieve array data available at address source on target PE (pe). The elements of the source array are separated by a stride sst. Once the data is received, it is stored at the local memory address target, separated by stride tst. The routines return when the data has been copied into the local target array.

\section{Return Values}

Upon return of this routine, the data object at address target will contain the data retrieved from the target memory address source.

\subsection{Atomic Memory fetch-and-operate Routines}

This section describes the OpenSHMEM Atomic fetch-op Routines. These routines allow operations on a symmetric object guaranteeing that another process will not update target between the time of the fetch and the update.

\subsection{1 shmem_swap}

\section{Summary}

Performs an atomic swap operation. 


\section{Synopsis}

$\mathrm{C} / \mathrm{C}++$ :

int shmem_int_swap (int *target, int value, int pe);

long shmem_long_swap(long *target, long value, int pe);

long shmem_swap (long *target, long value, int pe);

long long shmem_longlong_swap(long long *target, long long value, int pe);

float shmem_float_swap (float *target, float value, int pe);

double shmem_double_swap (double *target, double value, int pe);

Fortran:

INTEGER pe

INTEGER SHMEM_SWAP

ires = SHMEM_SWAP (target, value, pe)

INTEGER (KIND=4) SHMEM_INT4_SWAP

ires = SHMEM_INT4_SWAP (target, value, pe)

INTEGER (KIND=8) SHMEM_INT8_SWAP

ires = SHMEM_INT8_SWAP (target, value, pe)

REAL (KIND=4) SHMEM_REAL4_SWAP

res = SHMEM_REAL4_SWAP (target, value, pe)

REAL (KIND=8) SHMEM_REAL8_SWAP

res = SHMEM_REAL8_SWAP (target, value, pe)

\section{Parameters}

target Address of the symmetric data object to be updated on the target pe.

value Value to be atomically written to the target PE.

pe PE number of the target PE.

\section{Constraints}

- target must be the address of a symmetric data object. 
- If using $\mathrm{C} / \mathrm{C}++$, the type of target must match that implied in the Synopsis section. When calling from Fortran, the target data types are as follows:

- For SHMEM_INT4_SWAP() target must be of type Integer, with element size of 4 bytes.

- For SHMEM_INT8_SWAP() target must be of type Integer, with element size of 8 bytes.

- For SHMEM_REAL4_SWAP() target must be of type Real, with element size of 4 bytes.

- For SHMEM_REAL8_SWAP() target must be of type Real, with element size of 8 bytes.

- value must be the same type as target.

- This process must be carried out guaranteeing that it will not be interrupted by any other operation.

\section{Effect}

The atomic swap routines write value to address target on PE pe, and return the previous contents of target. The operation must be completed without the possibility of another process updating target between the time of the fetch and the update.

\section{Return Values}

Returns the previous value of target.

\subsection{2 shmem_cswap}

\section{Summary}

The conditional swap routines conditionally update a target data object on an arbitrary processing element (PE) and return the prior contents of the data object in one atomic operation.

\section{Synopsis}

$\mathrm{C} / \mathrm{C}++$ : 
int shmem_int_cswap (int *target, int cond, int value, int pe);

long shmem_long_cswap(long *target, long cond, long value, int pe);

long shmem_longlong_cswap(longlong *target, longlong cond, longlong value, int pe);

Fortran:

INTEGER pe

INTEGER (KIND=4) SHMEM_INT4_CSWAP

ires = SHMEM_INT4_CSWAP (target, cond, value, pe)

INTEGER (KIND=8) SHMEM_INT8_CSWAP

ires = SHMEM_INT8_CSWAP (target, cond, value, pe)

\section{Parameters}

target Address of the symmetric data object to be updated on the target pe.

cond cond is compared to the target target value.

value The value to be atomically written to the target $\mathrm{PE}$.

pe PE number of the target PE.

\section{Constraints}

- target must be the address of a symmetric data object.

- If using $\mathrm{C} / \mathrm{C}++$, the type of target must match that implied in the Synopsis section. When calling from Fortran, the target data types are as follows:

- For SHMEM_INT4_CSWAP() target must be of type Integer, with element size of 4 bytes.

- For SHMEM_INT8_CSWAP() target must be of type Integer, with element size of 8 bytes.

- value and cond must be the same type as target.

- This process must be carried out guaranteeing that it will not be interrupted by any other operation. 


\section{Effect}

The conditional swap routines write value to address target on PE pe, and return the previous contents of target. The replacement must occur only if cond is equal to target; otherwise target is left unchanged. In either case, the routine must return the initial value of target. The operation must be completed without the possibility of another process updating target between the time of the fetch and the update.

\section{Return Values}

Returns the initial value of target.

\subsection{3 shmem_fadd}

\section{Summary}

These routines perform an atomic fetch-and-add operation.

\section{Synopsis}

$\mathrm{C} / \mathrm{C}++$ :

int shmem_int_fadd(int *target, int value, int pe);

long shmem_long_fadd(long *target, long value, int pe);

long long shmem_longlong_fadd(long long *target, long long value, int pe);

Fortran:

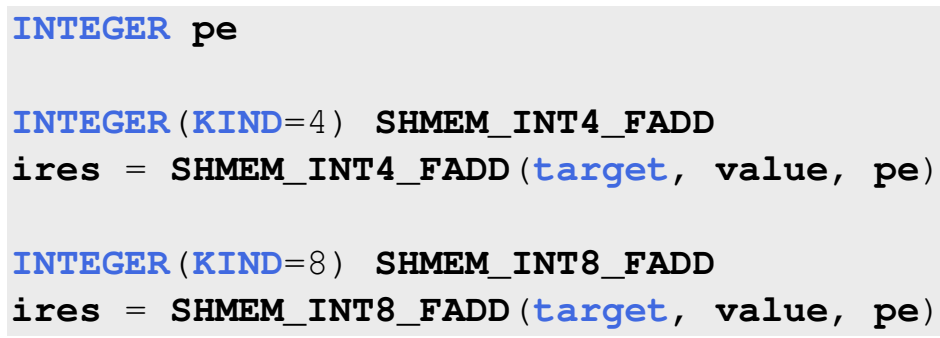

\section{Parameters}

target Address of the symmetric data object where to save the data on the target pe.

value The value to be atomically added to the value at address target.

pe PE number of the target PE. 


\section{Constraints}

- target must be the address of a symmetric data object.

- If using $\mathrm{C} / \mathrm{C}++$, the type of target must match that implied in the Synopsis section. When calling from Fortran, the target data types are as follows:

- For SHMEM_INT4_FADD() target must be of type Integer, with element size of 4 bytes.

- For SHMEM_INT8_FADD() target must be of type Integer, with element size of 8 bytes.

- value must be the same type as target.

- This process must be carried out guaranteeing that it will not be interrupted by any other operation.

\section{Effect}

The fetch and add routines retrieve the value at address target on PE pe, and update target with the result of adding value to the retrieved value. The operation must be completed without the possibility of another process updating target between the time of the fetch and the update.

\section{Return Values}

Returns the initial value of target.

\subsection{4 shmem_finc}

\section{Summary}

These routines perform a fetch-and-increment operation.

\section{Synopsis}

$\mathrm{C} / \mathrm{C}++$ :

int shmem_int_finc (int *target, int pe);

long shmem_long_finc(long *target, int pe);

long long shmem_longlong_finc(long long *target, int pe); 
Fortran:

INTEGER pe

INTEGER (KIND=4) SHMEM_INT4_FINC

ires = SHMEM_INT4_FINC (target 4, pe)

INTEGER (KIND=8) SHMEM_INT8_FINC

ires = SHMEM_INT8_FINC (target 8, pe $)$

\section{Parameters}

target Address of the symmetric data object where to save the data on the target pe. pe PE number of the target PE.

\section{Constraints}

- target must be the address of a symmetric data object.

- If using $\mathrm{C} / \mathrm{C}++$, the type of target must match that implied in the Synopsis section. When calling from Fortran, the target data types are as follows:

- For SHMEM_INT4_FINC() target must be of type Integer, with element size of 4 bytes.

- For SHMEM_INT8_FINC() target must be of type Integer, with element size of 8 bytes.

- This process must be carried out guaranteeing that it will not be interrupted by any other operation.

\section{Effect}

The fetch and increment routines retrieve the value at address target on PE pe, and update target with the result of incrementing the retrieved value by one. The operation must be completed without the possibility of another process updating target between the time of the fetch and the update.

\section{Return Values}

Returns the initial value of target. 


\subsection{Atomic Memory Operation Routines}

\subsection{1 shmem_add}

\section{Summary}

These routines perform an atomic add operation.

\section{Synopsis}

$\mathrm{C} / \mathrm{C}++$ :

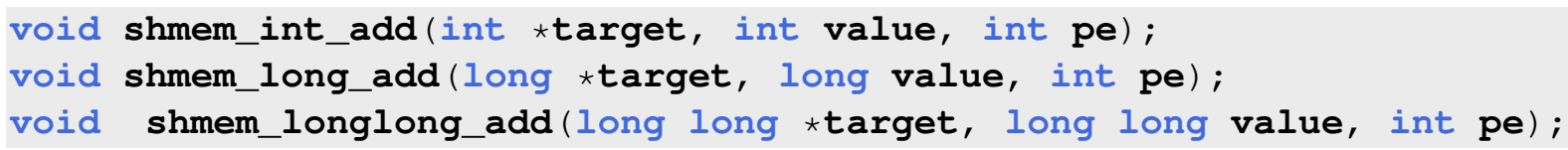

Fortran:

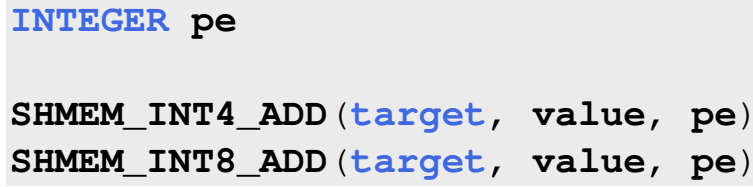

\section{Parameters}

target Address of the symmetric data object where to save the data on the target pe.

value The value to be atomically added to target.

pe An integer that indicates the PE number upon which target is to be updated. If you are using Fortran, it must be a default integer value.

\section{Constraints}

- target must be the address of a symmetric data object.

- If using $\mathrm{C} / \mathrm{C}++$, the type of var must match that implied in the Synopsis section. When calling from Fortran, the data type of var must be as follows:

- For SHMEM_INT4_ADD(), var must be of type Integer, with element size of 4 bytes. 
- For SHMEM_INT8_ADD(), var must be of type Integer, with element size of 8 bytes.

- value must be the same type as target.

- This process must be carried out guaranteeing that it will not be interrupted by any other operation.

\section{Effect}

The atomic add routines add value to the data at address target on PE pe. The operation must be completed without the possibility of another process updating target between the time of the fetch and the update.

\section{Return Values}

None.

\subsection{2 shmem_inc}

\section{Summary}

These routines perform an atomic increment operation on a target data object.

\section{Synopsis}

$\mathrm{C} / \mathrm{C}++$ :

void shmem_int_inc(int *target, int pe);

void shmem_long_inc(long *target, int pe);

void shmem_longlong_inc(long long *target, int pe);

Fortran:

INTEGER pe

INTEGER (KIND $=4)$ target 4

CALI SHMEM_INT4_INC (target4, pe)

INTEGER (KIND=8) target 8

CALI SHMEM_INT8_INC (target8, pe) 


\section{Parameters}

target Address of the symmetric data object where to save the data on the target pe. pe PE number of the target PE.

\section{Constraints}

- target must be the address of a symmetric data object.

- If using $\mathrm{C} / \mathrm{C}++$, the type of target must match that implied in the Synopsis section. When calling from Fortran, the target data types are as follows:

- For SHMEM_INT4_INC() target must be of type Integer, with element size of 4 bytes.

- For SHMEM_INT8_INC() target must be of type Integer, with element size of 8 bytes.

- This process must be carried out guaranteeing that it will not be interrupted by any other operation.

\section{Effect}

The atomic increment routines replace the value of target with its value incremented by one. The operation must be completed without the possibility of another process updating target between the time of the fetch and the update.

\section{Return Values}

None.

\subsection{Point-to-Point Synchronization Routines}

The point-to-point synchronization routines force the calling PE to halt execution until a symmetric data object is changed by a target write or atomic swap issued by another PE.

\subsection{1 shmem_wait}

\section{Summary}

These routines force the calling PE to wait until var is no longer equal to value. 


\section{Synopsis}

$\mathrm{C} / \mathrm{C}++$ :

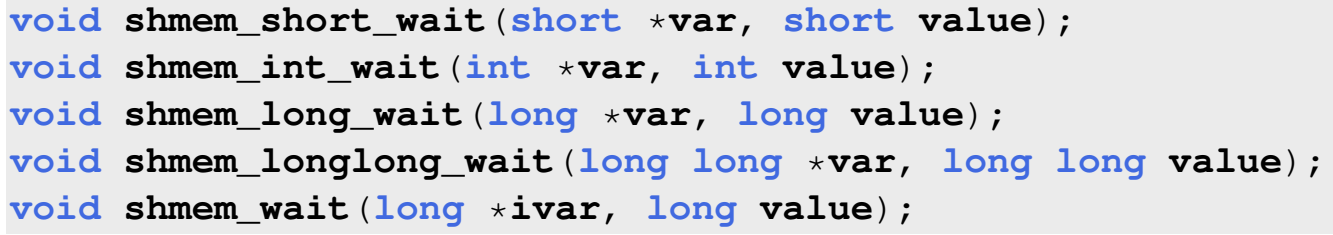

Fortran:

CALI SHMEM_INT4_WAIT (var, value)
CALI SHMEM_INT8_WAIT (var, value)
CALL SHMEM_WAIT (var, value)

\section{Parameters}

var The symmetric data object to be monitored on the calling PE.

value Value to be compared against the value at address var.

\section{Constraints}

- var must be the address of a symmetric data object.

- If using $\mathrm{C} / \mathrm{C}++$, the type of var must match that implied in the Synopsis section. When calling from Fortran, the data type of var must be as follows:

- For SHMEM_INT4_WAIT(), var must be of type Integer, with element size of 4 bytes.

- For SHMEM_INT8_WAIT(), var must be of type Integer, with element size of 8 bytes.

- value must be the same type as var.

\section{Effect}

A call to any shmem_wait() routine does not return until some other processor makes the value at address var not equal to value. 


\section{Return Values}

None.

\subsection{2 shmem_wait_until}

\section{Summary}

These routines force the calling PE to wait until the condition indicated by cond and value is satisfied.

\section{Synopsis}

$\mathrm{C} / \mathrm{C}++$ :

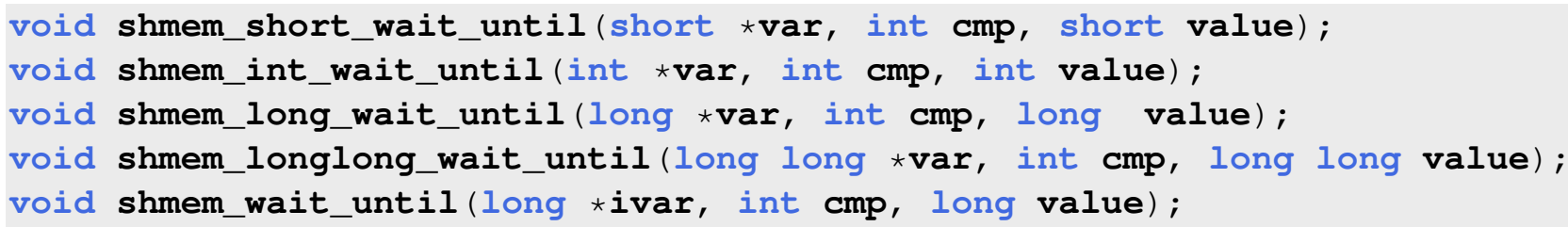

Fortran:

INTEGER cmp

CALI SHMEM_INT4_WAIT_UNTIL (var, cmp, value)

CALL SHMEM_INT8_WAIT_UNTIL (var, cmp, value)

CALI SHMEM_WAIT_UNTIL (var, cmp, value)

\section{Parameters}

var The symmetric data object to be monitored on the calling PE.

$\mathrm{cmp}$ Indicates how to compare value at address var and value. The following are the allowed compare operations:

SHMEM_CMP_EQ Equal

SHMEM_CMP_NE Not equal

SHMEM_CMP_GT Greater than

SHMEM_CMP_LE Less than or equal to

SHMEM_CMP_LT Less than 
SHMEM_CMP_GE Greater than or equal to

value Value to be compared against the value at address var.

\section{Constraints}

- var must be the address of a symmetric data object.

- If using $\mathrm{C} / \mathrm{C}++$, the type of var must match that implied in the Synopsis section. When calling from Fortran, the data type of var must be as follows:

- For SHMEM_INT4_WAIT_UNTIL(), var must be of type Integer, with element size of 4 bytes.

- For SHMEM_INT8_WAIT_UNTIL(), var must be of type Integer, with element size of 8 bytes.

- value must be the same type as var.

- If using $\mathrm{C} / \mathrm{C}++$ it must be of integer type. If you are using Fortran, $\mathbf{c m p}$ must be of default kind.

\section{Effect}

A call to any shmem_wait_until() routine does not return until some other processor changes the value at address var to satisfy the condition implied by $\mathbf{c m p}$ and value.

\section{Return Values}

None.

\subsection{Barrier Synchronization Routines}

\subsection{1 shmem_barrier_all}

\section{Summary}

Suspends the execution of the calling PE until all other PEs issue a call to this particular shmem_barrier_all() statement. 


\section{Synopsis}

$\mathrm{C} / \mathrm{C}++$ :

void shmem_barrier_all (void) ;

Fortran:

CALI SHMEM_BARRIER_ALL ( )

\section{Parameters}

None.

\section{Constraints}

- All PEs must call this routine at the same point of the execution.

\section{Effect}

The shmem_barrier_all() routine does not return until all other PEs have entered this routine at the same point of the execution path.

Prior to synchronizing with other PEs, shmem_barrier_all() ensures completion of all previously issued local memory stores and target memory updates issued via OpenSHMEM functions such as shmem_put32.

\section{Return Values}

None.

\subsection{2 shmem_barrier}

\section{Summary}

Performs a barrier operation on a subset of processing elements (PEs). 


\section{Synopsis}

$\mathrm{C} / \mathrm{C}++$ :

void shmem_barrier(int PE_start, int logPE_stride, int PE_size, long $\star$ pSync) ;

Fortran:

INTEGER PE_start, $\log P E \_s t r i d e, \quad P E \_s i z e$

INTEGER PSYNC (SHMEM_BARRIER_SYNC_SIZE)

CALI SHMEM_BARRIER(PE_start, logPE_stride, PE_size, pSync)

\section{Parameters}

PE_start The lowest PE number of the active set of PEs.

logPE_stride The log (base 2) of the stride between consecutive PE numbers in the active set.

PE_size The number of PEs in the active set.

pSync A symmetric work array.

\section{Constraints}

- If using $\mathrm{C} / \mathrm{C}++$, PE_start must be of type integer. If you are using Fortran, it must be a default integer value. Its value must be greater than or equal to zero.

- If using $\mathrm{C} / \mathrm{C}++$, logPE_stride must be of type integer. If you are using Fortran, it must be a default integer value.

- If using $\mathrm{C} / \mathrm{C}++$, PE_size must be of type integer. If you are using Fortran, it must be a default integer value. Its value must be less than or equal to the total number of PEs minus one.

- In $\mathrm{C} / \mathrm{C}++$, pSync must be type integer and of size _SHMEM_BARRIER_SYNC_SIZE. In Fortran, pSync must be of type integer and size SHMEM_BARRIER_SYNC_SIZE. If you are using Fortran, it must be a default integer type. Every element of this array must be initialized to _SHMEM_SYNC_VALUE (SHMEM_SYNC_VALUE in Fortran) before any of the PEs in the active set enter shmem_barrier the first time. 


\section{Effect}

The shmem_barrier() routine does not return until the subset of PEs specified by PE_start, logPE_stride and PE_size, have entered this routine at the same point of the execution path.

\section{Notes}

If the pSync array is initialized at run time, be sure to use some type of synchronization, for example, a call to shmem_barrier_all before calling shmem_barrier for the first time.

If the active set does not change, shmem_barrier can be called repeatedly with the same pSync array. No additional synchronization beyond that implied by shmem_barrier itself is necessary in this case.

\section{Return Values}

None.

\subsection{3 shmem_fence}

\section{Summary}

Provides a separate ordering on the sequence of puts issued by this PE to each destination PE.

\section{Synopsis}

$\mathrm{C} / \mathrm{C}++$ :

void shmem_fence (void);

Fortran:

CALI SHMEM_FENCE

\section{Parameters}

None. 


\section{Constraints}

None.

\section{Effect}

The shmem_fence() routine provides an ordering on the put operations issued by the calling PE prior to the call to shmem_fence() relative to the put operations issued by the calling PE following the call to shmem_fence(). It guarantees that all such prior put operations issued to a particular destination PE are fully written to the symmetric memory of that destination PE, before any such following put operations to that same destination PE are written to the symmetric memory of that destination PE.

Note that the ordering is provided separately on the sequences of puts from the calling PE to each distinct destination PE. The shmem_quiet() routine should be used instead if ordering of puts is required when multiple destination PEs are involved.

\section{Return Values}

None.

\subsection{4 shmem_quiet}

\section{Summary}

Provides an ordering on the sequence of puts issued by this PE across all destination PEs.

\section{Synopsis}

$\mathrm{C} / \mathrm{C}++$ :

void shmem_quiet (void);

Fortran:

CALL SHMEM_QUIET

\section{Parameters}

None. 


\section{Constraints}

None.

\section{Effect}

The shmem_quiet() routine provides an ordering on the put operations issued by the calling PE prior to the call to shmem_quiet() relative to memory load, memory store, put, get or synchronization operations issued by the calling PE following the call to shmem_quiet(). It guarantees that all such prior put operations are fully written to the symmetric memory of the destination PE and visible to all other PEs, before any such following memory load, memory store, put, get or synchronization operations are visible to any PE.

\section{Return Values}

None.

\subsection{Reduction Routines}

The OpenSHMEM reduction routines perform an associative binary operation across symmetric arrays on multiple PEs. See Figure 3 for a generalized diagram of reduction operations. 


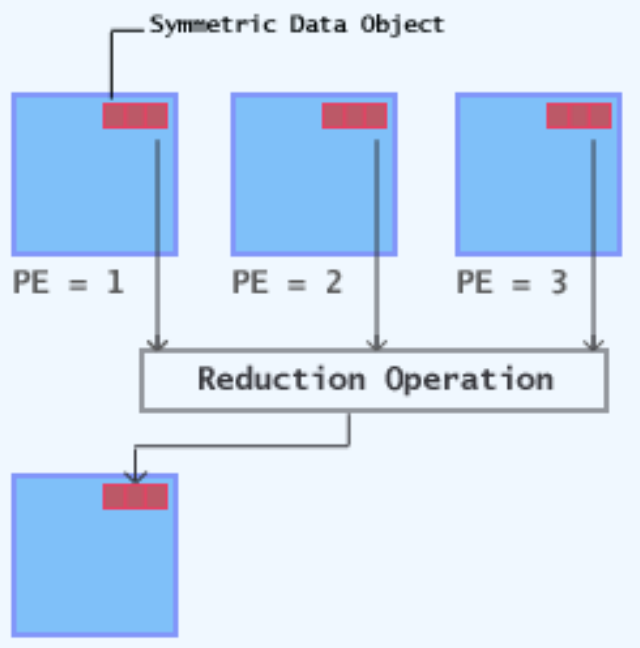

$\mathrm{PE}=0$

(Calling PE )

Fig. 3: General diagram of reduction operations

\subsection{1 shmem_and}

\section{Summary}

Performs a bitwise AND operation on symmetric arrays over the active set of PEs.

\section{Synopsis}

$\mathrm{C} / \mathrm{C}++$ :

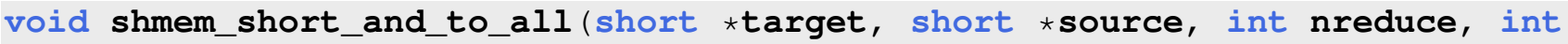

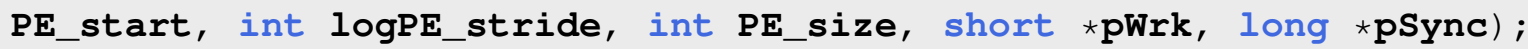
void shmem_int_and_to_all (int *target, int *source, int nreduce, int PE_start, int logPE_stride, int PE_size, int *pWrk, long *pSync); void shmem_long_and_to_all(long *target, long $\star$ source, int nreduce, int PE_start, int logPE_stride, int PE_size, long $\star$ pWrk, long $\star$ pSync);

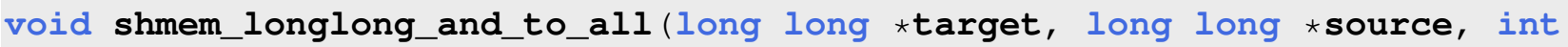
nreduce, int PE_start, int logPE_stride, int PE_size, long long $\star$ pWrk, long $\star$ pSync);

Fortran: 


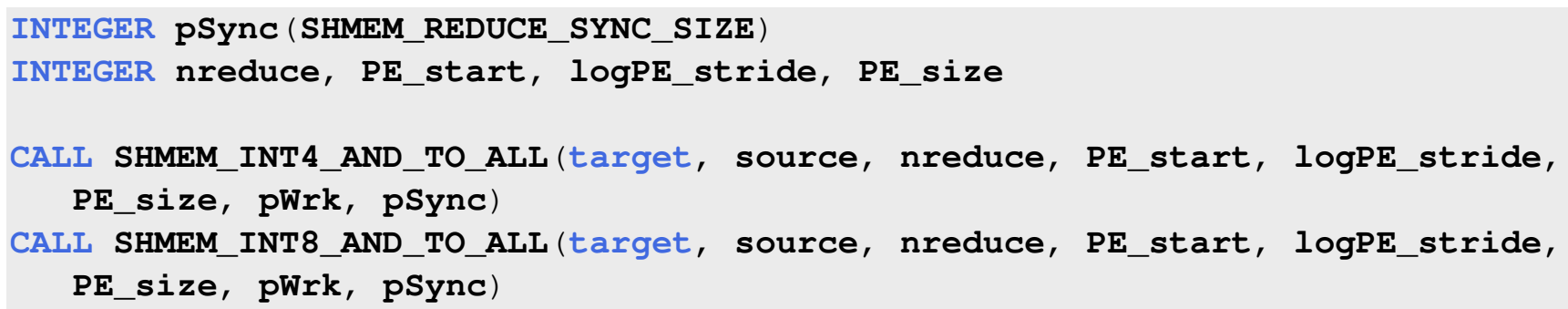

\section{Parameters}

target Address of the symmetric data object where to store the results of the reduction operation.

source Address of the symmetric data object that contains the elements for each separate reduction operation.

nreduce The number of elements in the target and source arrays.

PE_start The lowest PE number of the active set of PEs.

logPE_stride The log (base 2) of the stride between consecutive PE numbers in the active set.

PE_size The number of PEs in the active set.

pWrk A symmetric work array.

pSync A symmetric work array.

\section{Constraints}

- target and source must be the addresses of symmetric data objects.

- The target array on all PEs in the active set must be ready to accept the results of the reduction.

- If using $\mathrm{C} / \mathrm{C}++$, the type of target must match that implied in the Synopsis section. When calling from Fortran, the data type of target must be as follows:

- For SHMEM_INT4_AND_TO_ALL(), var must be of type Integer, with element size of 4 bytes.

- For SHMEM_INT8_AND_TO_ALL(), var must be of type Integer, with element size of 8 bytes. 
- source must be the same type as target.

- source and target may be the same array, but they must not be overlapping arrays.

- If using $\mathrm{C} / \mathrm{C}++$, nreduce must be of type integer. If you are using Fortran, it must be a default integer value.

- If using $\mathrm{C} / \mathrm{C}++$, PE_start must be of type integer. If you are using Fortran, it must be a default integer value. Its value must be greater than or equal to zero.

- If using $\mathrm{C} / \mathrm{C}++$, logPE_stride must be of type integer. If you are using Fortran, it must be a default integer value.

- If using $\mathrm{C} / \mathrm{C}++$, PE_size must be of type integer. If you are using Fortran, it must be a default integer value. Its value must be less than or equal to the total number of PEs minus one.

- The pWrk argument must have the same data type as target. In $\mathrm{C} / \mathrm{C}++$, this contains max(nreduce/2 + 1, SHMEM_REDUCE_MIN_WRKDATA_SIZE) elements. In Fortran, this contains $\max (\mathbf{n} \overline{r e d u c e} / \overline{2}+1$, SHMEM_REDUCE_MIN_WRKDATA_SIZE) elements.

- In $\mathrm{C} / \mathrm{C}++$, pSync must be of type long and size _SHMEM_REDUCE_SYNC_SIZE. In Fortran, pSync must be of type integer and size SHMEM_REDUCE_SYNC_SIZE. If you are using Fortran, it must be a default integer value. Every element of this array must be initialized with the value _SHMEM_SYNC_VALUE (in C/C++) or SHMEM_SYNC_ VALUE (in Fortran) before any of the PEs in the active set enter the reduction routine.

- The pWrk and pSync arrays on all PEs in the active set must not be in use from a prior call to a collective OpenSHMEM routine.

- This routine must be called by all the PEs in the active set at the same point of the execution path; otherwise undefined behavior results.

\section{Effect}

This routine returns the result of performing a bitwise AND operation on the source data object of every PE in the active set. The active set of PEs is defined by the triple PE_start, logPE_stride and PE_size.

As with all OpenSHMEM collective routines, each of these routines assumes that only PES in the active set call the routine. If a PE not in the active set calls a OpenSHMEM collective routine, undefined behavior results. 


\section{Notes}

All OpenSHMEM reduction routines reset the values in pSync before they return, so a particular pSync buffer need only be initialized the first OpenSHMEM reduction routine. Be careful of the following situations: If the pSync array is initialized at run time, some type of synchronization is needed to ensure that all PEs in the working set have initialized pSync before any of them enter a OpenSHMEM routine called with the pSync synchronization array. A pSync or pWrk array can be reused in a subsequent reduction routine call only if none of the PEs in the active set are still processing a prior reduction routine call that used the same pSync or pWrk arrays. In general, this can be assured only by doing some type of synchronization. However, in the special case of reduction routines being called with the same active set, you can allocate two pSync and pWrk arrays and alternate between them on successive calls.

\section{Return Values}

Upon completion, the target array will be updated with the result of the bitwise AND operation and the elements of the pSync array will be restored to their initial values.

\subsection{2 shmem_or}

\section{Summary}

Performs a bitwise OR operation on symmetric arrays over the active set of PEs.

\section{Synopsis}

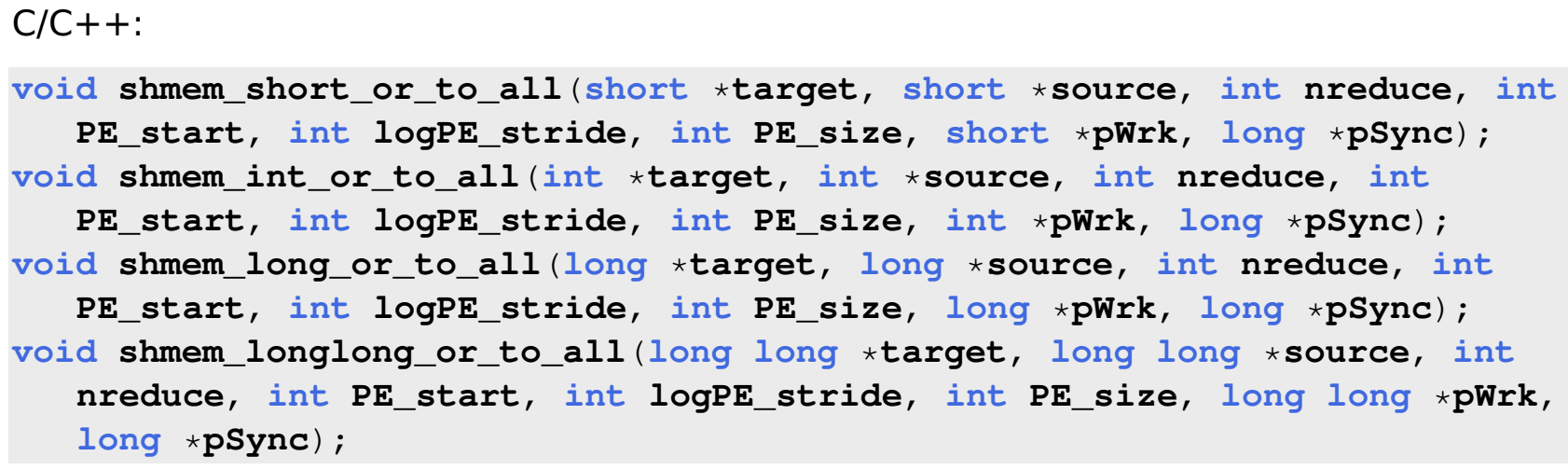

Fortran: 


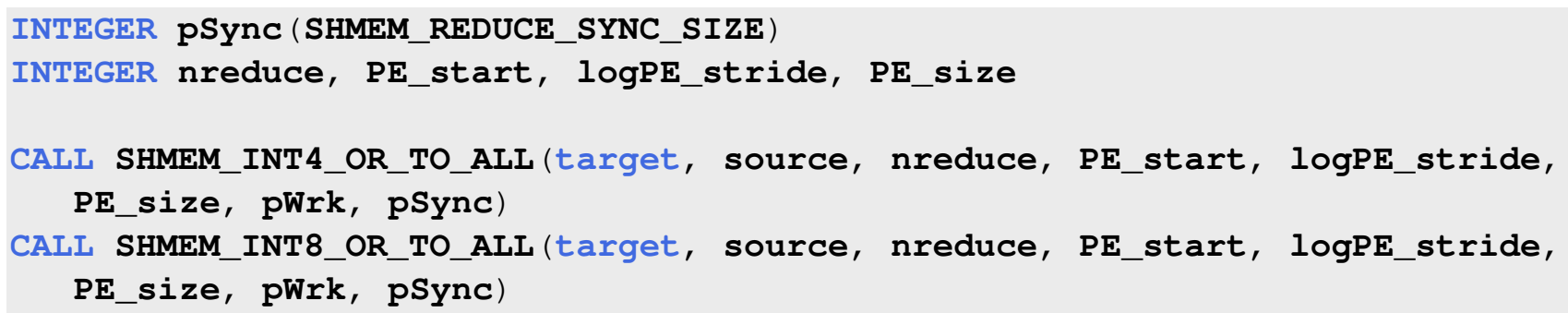

\section{Parameters}

target Address of the symmetric data object where to store the results of the reduction operation.

source Address of the symmetric data object that contains the elements for each separate reduction operation.

nreduce The number of elements in the target and source arrays.

PE_start The lowest PE number of the active set of PEs.

logPE_stride The log (base 2) of the stride between consecutive PE numbers in the active set.

PE_size The number of PEs in the active set.

pWrk A symmetric work array.

pSync A symmetric work array.

\section{Constraints}

- The target array on all PEs in the active set must be ready to accept the results of the reduction.

- target and source must be the addresses of symmetric data objects.

- If using $\mathrm{C} / \mathrm{C}++$, the type of target must match that implied in the Synopsis section. When calling from Fortran, the data type of target must be as follows:

- For SHMEM_INT4_OR_TO_ALL(), target must be of type Integer, with element size of 4 bytes.

- For SHMEM_INT8_OR_TO_ALL(), target must be of type Integer, with element size of 8 bytes. 
- source must be the same type as target.

- source and target may be the same array, but they must not be overlapping arrays.

- If using $\mathrm{C} / \mathrm{C}++$, nreduce must be of type integer. If you are using Fortran, it must be a default integer value.

- If using $\mathrm{C} / \mathrm{C}++$, PE_start must be of type integer. If you are using Fortran, it must be a default integer value. Its value must be greater than or equal to zero.

- If using $\mathrm{C} / \mathrm{C}++$, logPE_stride must be of type integer. If you are using Fortran, it must be a default integer value.

- If using $\mathrm{C} / \mathrm{C}++$, PE_size must be of type integer. If you are using Fortran, it must be a default integer value. Its value must be less than or equal to the total number of PEs minus one.

- The pWrk argument must have the same data type as target. In $\mathrm{C} / \mathrm{C}++$, this contains max(nreduce/2 + 1, SHMEM_REDUCE_MIN_WRKDATA_SIZE) elements. In Fortran, this contains $\max (\mathbf{n} \overline{r e d u c e} / \overline{2}+1$, SHMEM_REDUCE_MIN_WRKDATA_SIZE) elements.

- In $\mathrm{C} / \mathrm{C}++$, pSync must be of type long and size _SHMEM_REDUCE_SYNC_SIZE. In Fortran, pSync must be of type integer and size SHMEM_REDUCE_SYNC_SIZE. If you are using Fortran, it must be a default integer value. Every element of this array must be initialized with the value _SHMEM_SYNC_VALUE (in C/C++) or SHMEM_SYNC_ VALUE (in Fortran) before any of the PEs in the active set enter the reduction routine.

- The pWrk and pSync arrays on all PEs in the active set must not be in use from a prior call to a collective OpenSHMEM routine.

- This routine must be called by all the PEs in the active set at the same point of the execution path; otherwise undefined behavior results.

\section{Effect}

This routine returns the result of performing a bitwise OR operation on the source data object of every PE in the active set. The active set of PEs is defined by the triple PE_start, logPE_stride and PE_size.

As with all OpenSHMEM collective routines, each of these routines assumes that only PES in the active set call the routine. If a PE not in the active set calls a OpenSHMEM collective routine, undefined behavior results. 


\section{Notes}

All OpenSHMEM reduction routines reset the values in pSync before they return, so a particular pSync buffer need only be initialized the first OpenSHMEM reduction routine. Be careful of the following situations: If the pSync array is initialized at run time, some type of synchronization is needed to ensure that all PEs in the working set have initialized pSync before any of them enter a OpenSHMEM routine called with the pSync synchronization array. A pSync or pWrk array can be reused in a subsequent reduction routine call only if none of the PEs in the active set are still processing a prior reduction routine call that used the same pSync or pWrk arrays. In general, this can be assured only by doing some type of synchronization. However, in the special case of reduction routines being called with the same active set, you can allocate two pSync and pWrk arrays and alternate between them on successive calls.

\section{Return Values}

Upon completion, the target array will be updated with the result of the bitwise OR operation and the elements of the pSync array will be restored to their initial values.

\subsection{3 shmem_xor}

\section{Summary}

Performs a bitwise XOR operation on symmetric arrays over the active set of PEs. The active set of PEs is defined by the triple PE_start, logPE_stride and PE_size.

\section{Synopsis}

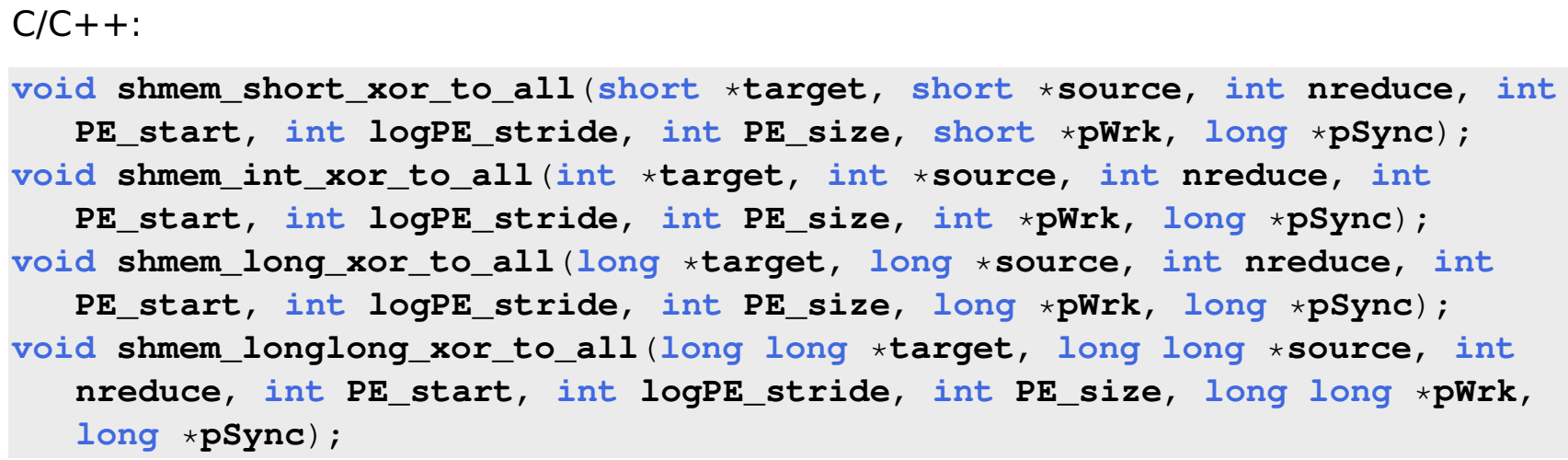

Fortran: 


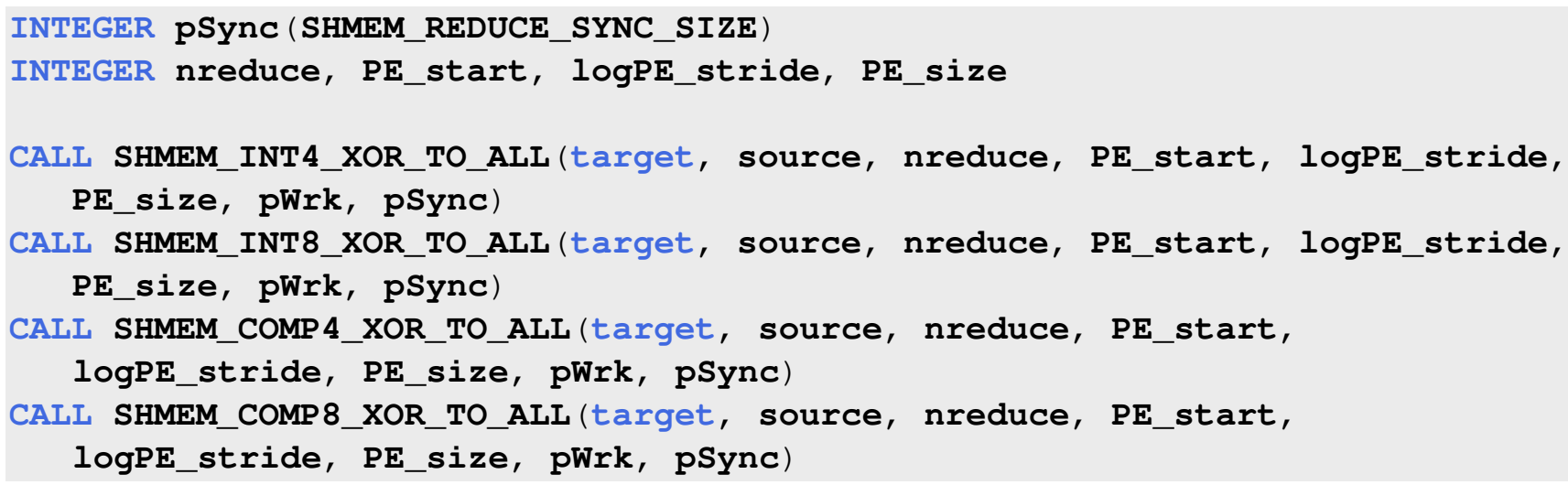

\section{Parameters}

target Address of the symmetric data object where to store the results of the reduction operation.

source Address of the symmetric data object that contains the elements for each separate reduction operation.

nreduce The number of elements in the target and source arrays.

PE_start The lowest PE number of the active set of PEs.

logPE_stride The log (base 2) of the stride between consecutive PE numbers in the active set.

PE_size The number of PEs in the active set.

pWrk A symmetric work array.

pSync A symmetric work array.

\section{Constraints}

- The target array on all PEs in the active set must be ready to accept the results of the reduction.

- target and source must be the addresses of symmetric data objects.

- If using $\mathrm{C} / \mathrm{C}++$, the type of target must match that implied in the Synopsis section. When calling from Fortran, the data type of target must be as follows: 
- For SHMEM_INT4_XOR_TO_ALL(), target must be of type Integer, with element size of 4 bytes.

- For SHMEM_INT8_XOR_TO_ALL(), target must be of type Integer, with element size of 8 bytes.

- source must be the same type as target.

- source and target may be the same array, but they must not be overlapping arrays.

- If using $\mathrm{C} / \mathrm{C}++$, nreduce must be of type integer. If you are using Fortran, it must be a default integer value.

- If using $\mathrm{C} / \mathrm{C}++$, PE_start must be of type integer. If you are using Fortran, it must be a default integer value. Its value must be greater than or equal to zero.

- If using $\mathrm{C} / \mathrm{C}++$, logPE_stride must be of type integer. If you are using Fortran, it must be a default integer value.

- If using $\mathrm{C} / \mathrm{C}++$, PE_size must be of type integer. If you are using Fortran, it must be a default integer value. Its value must be less than or equal to the total number of PEs minus one.

- The pWrk argument must have the same data type as target. In $\mathrm{C} / \mathrm{C}++$, this contains max(nreduce/2 + 1, SHMEM_REDUCE_MIN_WRKDATA_SIZE) elements. In Fortran, this contains $\max (\mathbf{n} \bar{r} e d u c e / 2+1$, SHMEM_REDUCE_MIN_WRKDATA_SIZE) elements.

- In $\mathrm{C} / \mathrm{C}++$, pSync must be of type long and size _SHMEM_REDUCE_SYNC_SIZE. In Fortran, pSync must be of type integer and size SHMEM_REDUCE_SYNC_SIZ̈E. If you are using Fortran, it must be a default integer value. Every element of this array must be initialized with the value _SHMEM_SYNC_VALUE (in C/C++) or SHMEM_SYNC_ VALUE (in Fortran) before any of the PEs in the active set enter the reduction routine.

- The pWrk and pSync arrays on all PEs in the active set must not be in use from a prior call to a collective OpenSHMEM routine.

- This routine must be called by all the PEs in the active set at the same point of the execution path; otherwise undefined behavior results.

\section{Effect}

This routine returns the result of performing a bitwise XOR operation on the source data object of every PE in the active set. The active set of PEs is defined by the triple PE_start, logPE_stride and PE_size. 
As with all OpenSHMEM collective routines, each of these routines assumes that only PEs in the active set call the routine. If a PE not in the active set calls a OpenSHMEM collective routine, undefined behavior results.

\section{Notes}

All OpenSHMEM reduction routines reset the values in pSync before they return, so a particular pSync buffer need only be initialized the first OpenSHMEM reduction routine. Be careful of the following situations: If the pSync array is initialized at run time, some type of synchronization is needed to ensure that all PEs in the working set have initialized pSync before any of them enter a OpenSHMEM routine called with the pSync synchronization array. A pSync or pWrk array can be reused in a subsequent reduction routine call only if none of the PEs in the active set are still processing a prior reduction routine call that used the same pSync or pWrk arrays. In general, this can be assured only by doing some type of synchronization. However, in the special case of reduction routines being called with the same active set, you can allocate two pSync and pWrk arrays and alternate between them on successive calls.

\section{Return Values}

Upon completion, the target array will be updated with the result of the bitwise XOR operation and the elements of the pSync array will be restored to their initial values.

\subsection{4 shmem_max}

\section{Summary}

Computes the maximum value of the source symmetric array over the active set of PEs.

\section{Synopsis}

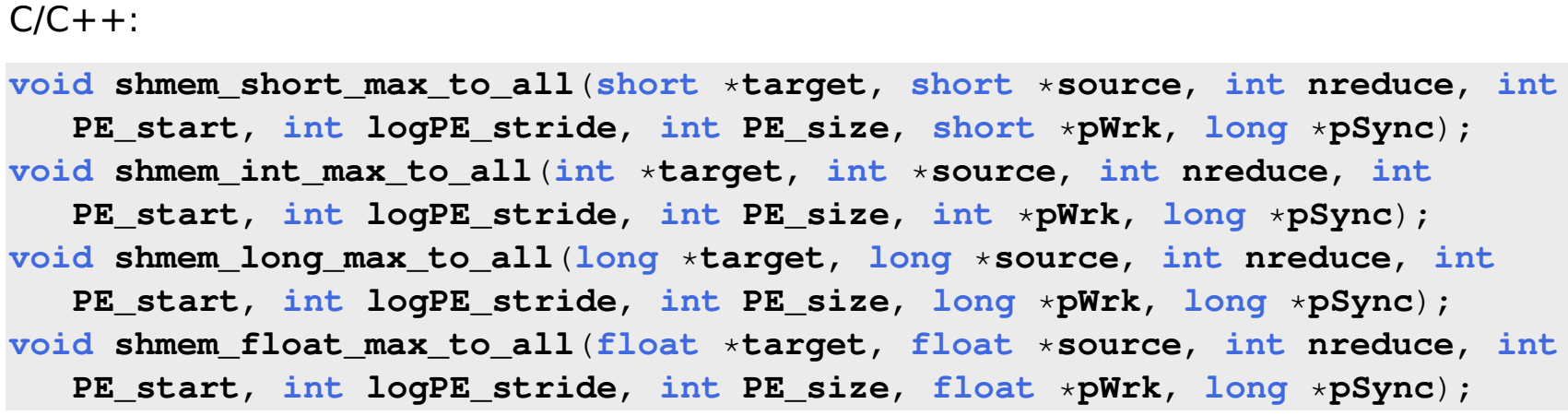




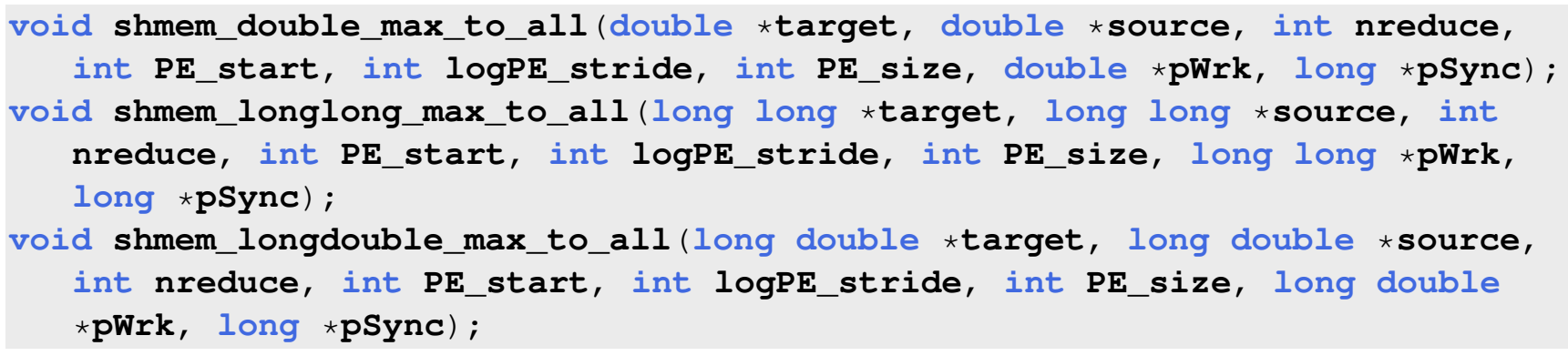

Fortran:

INTEGER PSYNC (SHMEM_REDUCE_SYNC_SIZE)

INTEGER nreduce, PE_start, logPE_stride, PE_size

CALL SHMEM_INT4_MAX_TO_ALL(target, source, nreduce, PE_start, logPE_stride, PE_size, pWrk, pSync)

CALL SHMEM_INT8_MAX_TO_ALL(target, source, nreduce, PE_start, logPE_stride, PE_size, pWrk, pSync)

CALI SHMEM_REAL4_MAX_TO_ALL (target, source, nreduce, PE_start, logPE_stride, PE_size, pWrk, pSync)

CALL SHMEM_REAL8_MAX_TO_ALI (target, source, nreduce, PE_start, logPE_stride, PE_size, pWrk, pSync)

CALL SHMEM_REAL16_MAX_TO_ALL (target, source, nreduce, PE_start, logPE_stride, PE_size, pWrk, pSync)

\section{Parameters}

target Address of the symmetric data object where to store the results of the reduction operation.

source Address of the symmetric data object that contains the elements for each separate reduction operation.

nreduce The number of elements in the target and source arrays.

PE_start The lowest PE number of the active set of PEs.

logPE_stride The log (base 2) of the stride between consecutive PE numbers in the active set.

PE_size The number of PEs in the active set.

pWrk A symmetric work array.

pSync A symmetric work array. 


\section{Constraints}

- The target array on all PEs in the active set must be ready to accept the results of the reduction.

- target and source must be the addresses of symmetric data objects.

- If using $\mathrm{C} / \mathrm{C}++$, the type of target must match that implied in the Synopsis section. When calling from Fortran, the data type of target must be as follows:

- For SHMEM_INT4_MAX_TO_ALL(), target must be of type Integer, with element size of 4 bytes.

- For SHMEM_INT8_MAX_TO_ALL(), target must be of type Integer, with element size of 8 bytes.

- For SHMEM_REAL4_MAX_TO_ALL(), target must be of type Real, with element size of 4 bytes.

- For SHMEM_REAL8_MAX_TO_ALL(), target must be of type Real, with element size of 8 bytes.

- For SHMEM_REAL16_MAX_TO_ALL(), target must be of type Real, with element size of 16 bytes.s

- source must be the same type as target.

- source and target may be the same array, but they must not be overlapping arrays.

- If using $\mathrm{C} / \mathrm{C}++$, nreduce must be of type integer. If you are using Fortran, it must be a default integer value.

- If using $\mathrm{C} / \mathrm{C}++$, PE_start must be of type integer. If you are using Fortran, it must be a default integer value. Its value must be greater than or equal to zero.

- If using $\mathrm{C} / \mathrm{C}++$, logPE_stride must be of type integer. If you are using Fortran, it must be a default integer value.

- If using $\mathrm{C} / \mathrm{C}++$, PE_size must be of type integer. If you are using Fortran, it must be a default integer value. Its value must be less than or equal to the total number of PEs minus one.

- The pWrk argument must have the same data type as target. In $\mathrm{C} / \mathrm{C}++$, this contains max(nreduce/2 + 1, SHMEM_REDUCE_MIN_WRKDATA_SIZE) elements. In Fortran, this contains $\max (\mathbf{n r e d u c e} / \overline{2}+1$, SHMEM_REDUCE_MIN_WRKDATA_SIZE) elements. 
- In $\mathrm{C} / \mathrm{C}++$, pSync must be of type long and size _SHMEM_REDUCE_SYNC_SIZE. In Fortran, pSync must be of type integer and size SHMEM_REDUCE_SYNC_SIZE. If you are using Fortran, it must be a default integer value. Every element of this array must be initialized with the value _SHMEM_SYNC_VALUE (in C/C++) or SHMEM_SYNC_ VALUE (in Fortran) before any of the PEs in the active set enter the reduction routine.

- The pWrk and pSync arrays on all PEs in the active set must not be in use from a prior call to a collective OpenSHMEM routine.

- This routine must be called by all the PEs in the active set at the same point of the execution path; otherwise undefined behavior results.

\section{Effect}

The max reduction routines determine the element with the highest value in array source across all PEs in the active set. The active set of PEs is defined by the triple PE_start, logPE_stride and PE_size. The results of the reduction must be stored at address target on all PEs of the active set.

As with all OpenSHMEM collective routines, each of these routines assumes that only PEs in the active set call the routine. If a PE not in the active set calls a OpenSHMEM collective routine, undefined behavior results.

\section{Notes}

All OpenSHMEM reduction routines reset the values in pSync before they return, so a particular pSync buffer need only be initialized the first OpenSHMEM reduction routine. Be careful of the following situations: If the pSync array is initialized at run time, some type of synchronization is needed to ensure that all PEs in the working set have initialized pSync before any of them enter a OpenSHMEM routine called with the pSync synchronization array. A pSync or pWrk array can be reused in a subsequent reduction routine call only if none of the PEs in the active set are still processing a prior reduction routine call that used the same pSync or pWrk arrays. In general, this can be assured only by doing some type of synchronization. However, in the special case of reduction routines being called with the same active set, you can allocate two pSync and pWrk arrays and alternate between them on successive calls.

\section{Return Values}

Upon completion, the target array will be updated with the result of the operation and the elements of the pSync array will be restored to their initial values. 


\subsection{5 shmem_min}

\section{Summary}

Computes the minimum value of symmetric arrays over the active set of PEs. The active set of PEs is defined by the triplet PE_start, logPE_stride and PE_size.

\section{Synopsis}

$\mathrm{C} / \mathrm{C}++$ :

void shmem_short_min_to_all (short *target, short $\star$ source, int nreduce, int

PE_start, int logPE_stride, int PE_size, short $\star$ pWrk, long $\star$ pSync); void shmem_int_min_to_all(int $\star$ target, int $\star$ source, int nreduce, int

PE_start, int logPE_stride, int PE_size, int $\star$ pWrk, long $\star$ pSync); void shmem_long_min_to_all(long $\star$ target, long $\star$ source, int nreduce, int

PE_start, int logPE_stride, int PE_size, long $\star$ pWrk, long $\star$ pSync) ;

void shmem_float_min_to_all(float *target, float *source, int nreduce, int

PE_start, int logPE_stride, int PE_size, float $\star$ pWrk, long $\star$ psync);

void shmem_double_min_to_all(double *target, double $\star$ source, int nreduce,

int PE_start, int logPE_stride, int PE_size, double $\star$ pWrk, long $\star$ pSync);

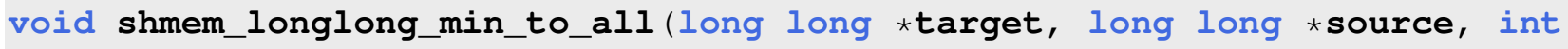

nreduce, int PE_start, int logPE_stride, int PE_size, long long $\star$ pWrk, long $\star$ pSync);

void shmem_longdouble_min_to_all(long double $\star$ target, long double $\star s o u r c e$,

int nreduce, int $\mathrm{PE} \_s t a r t$, int $\log \mathrm{PE} \_s t r i d e$, int $\mathrm{PE} \_s i z e$, long double $\star$ pWrk, long $\star$ pSync);

Fortran:

INTEGER PSYNC (SHMEM_REDUCE_SYNC_SIZE)

INTEGER nreduce, PE_start, logPE_stride, PE_size

CALI SHMEM_INT4_MIN_TO_ALL (target, source, nreduce, PE_start, logPE_stride, PE_size, pWrk, pSync)

CALL SHMEM_INT8_MIN_TO_ALL(target, source, nreduce, PE_start, logPE_stride, PE_size, pWrk, pSync)

CALI SHMEM_REAL4_MIN_TO_ALL (target, source, nreduce, PE_start, logPE_stride, PE_size, pWrk, pSync)

CALI SHMEM_REAL8_MIN_TO_ALL (target, source, nreduce, PE_start, logPE_stride, PE_size, pWrk, pSync)

CALI SHMEM_REAL16_MIN_TO_ALL (target, source, nreduce, PE_start, logPE_stride, PE_size, pWrk, pSync) 


\section{Parameters}

target Address of the symmetric data object where to store the results of the reduction operation.

source Address of the symmetric data object that contains the elements for each separate reduction operation.

nreduce The number of elements in the target and source arrays.

PE_start The lowest PE number of the active set of PEs.

logPE_stride The log (base 2) of the stride between consecutive PE numbers in the active set.

PE_size The number of PEs in the active set.

pWrk A symmetric work array.

pSync A symmetric work array.

\section{Constraints}

- The target array on all PEs in the active set must be ready to accept the results of the reduction.

- target and source must be the addresses of symmetric data objects.

- If using $\mathrm{C} / \mathrm{C}++$, the type of target must match that implied in the Synopsis section. When calling from Fortran, the data type of target must be as follows:

- For SHMEM_INT4_MIN_TO_ALL(), target must be of type Integer, with element size of 4 bytes.

- For SHMEM_INT8_MIN_TO_ALL(), target must be of type Integer, with element size of 8 bytes.

- For SHMEM_REAL4_MIN_TO_ALL(), target must be of type Real, with element size of 4 bytes.

- For SHMEM_REAL8_MIN_TO_ALL(), target must be of type Real, with element size of 8 bytes.

- For SHMEM_REAL16_MIN_TO_ALL(), target must be of type Real, with element size of 16 bytes.

- source must be the same type as target. 
- source and target may be the same array, but they must not be overlapping arrays.

- If using $\mathrm{C} / \mathrm{C}++$, nreduce must be of type integer. If you are using Fortran, it must be a default integer value.

- If using $\mathrm{C} / \mathrm{C}++$, PE_start must be of type integer. If you are using Fortran, it must be a default integer value. Its value must be greater than or equal to zero.

- If using $\mathrm{C} / \mathrm{C}++$, logPE_stride must be of type integer. If you are using Fortran, it must be a default integer value.

- If using $\mathrm{C} / \mathrm{C}++$, PE_size must be of type integer. If you are using Fortran, it must be a default integer value. Its value must be less than or equal to the total number of PEs minus one.

- The pWrk argument must have the same data type as target. In $\mathrm{C} / \mathrm{C}++$, this contains max(nreduce/2 + 1, SHMEM_REDUCE_MIN_WRKDATA_SIZE) elements. In Fortran, this contains $\max (\mathbf{n} r e d u c e / 2+1$, SHMEM_REDUCE_MIN_WRKDATA_SIZE) elements.

- In $\mathrm{C} / \mathrm{C}++$, pSync must be of type long and size _SHMEM_REDUCE_SYNC_SIZE. In Fortran, pSync must be of type integer and size SHMEM_REDUCE_SYNC_SIZEE. If you are using Fortran, it must be a default integer value. Every element of this array must be initialized with the value _SHMEM_SYNC_VALUE (in C/C++) or SHMEM_SYNC_ VALUE (in Fortran) before any of the PEs in the active set enter the reduction routine.

- The pWrk and pSync arrays on all PEs in the active set must not be in use from a prior call to a collective OpenSHMEM routine.

- This routine must be called by all the PEs in the active set at the same point of the execution path; otherwise undefined behavior results.

\section{Effect}

The min reduction routines determine the element with the lowest value in array source across all PEs in the active set. The active set of PEs is defined by the triple PE_start, logPE_stride and PE_size. The results of the reduction must be stored at address target on all PEs of the active set.

As with all OpenSHMEM collective routines, each of these routines assumes that only PEs in the active set call the routine. If a PE not in the active set calls a OpenSHMEM collective routine, undefined behavior results. 


\section{Notes}

All OpenSHMEM reduction routines reset the values in pSync before they return, so a particular pSync buffer need only be initialized the first OpenSHMEM reduction routine. Be careful of the following situations: If the pSync array is initialized at run time, some type of synchronization is needed to ensure that all PEs in the working set have initialized pSync before any of them enter a OpenSHMEM routine called with the pSync synchronization array. A pSync or pWrk array can be reused in a subsequent reduction routine call only if none of the PEs in the active set are still processing a prior reduction routine call that used the same pSync or pWrk arrays. In general, this can be assured only by doing some type of synchronization. However, in the special case of reduction routines being called with the same active set, you can allocate two pSync and pWrk arrays and alternate between them on successive calls.

\section{Return Values}

Upon completion, the target array will be updated with the result of the operation and the elements of the pSync array will be restored to their initial values.

\subsection{6 shmem_sum}

\section{Summary}

Computes the summation of symmetric arrays over the active set of PEs.

\section{Synopsis}

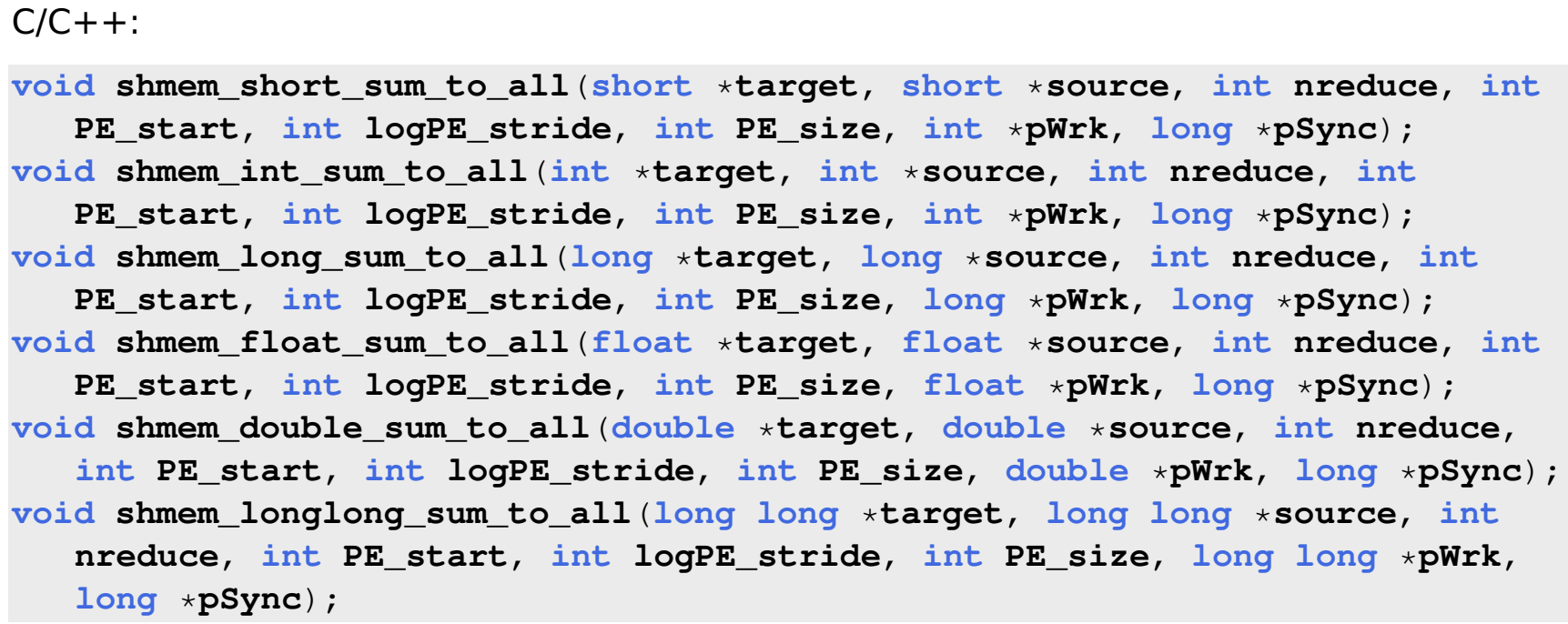




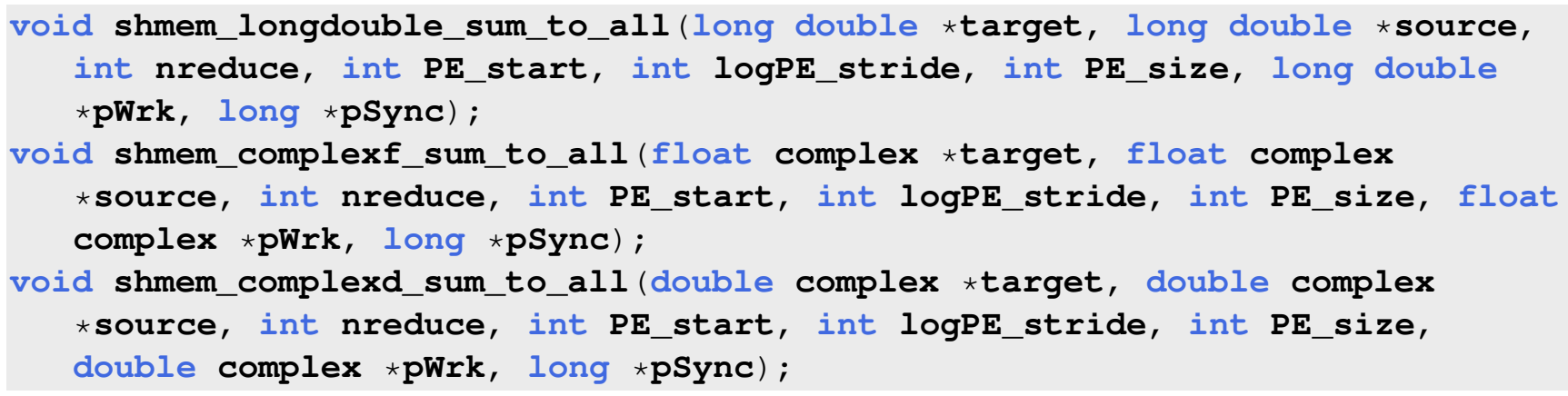

Fortran:

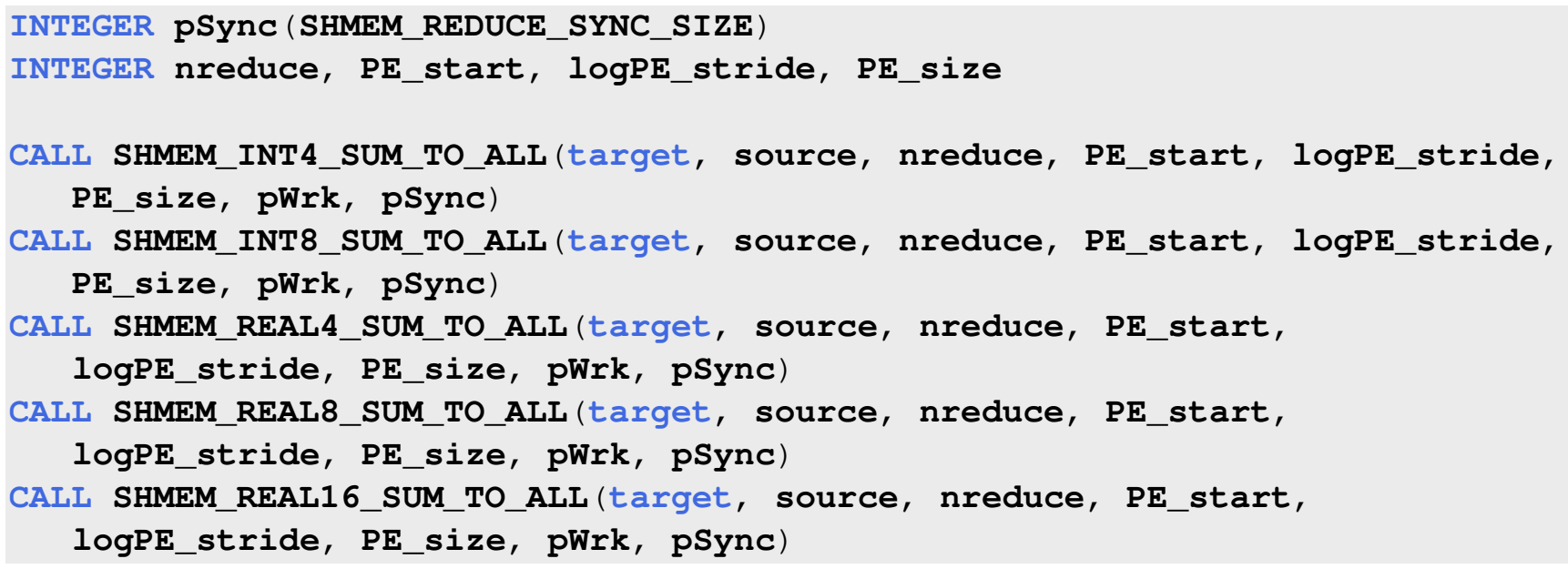

\section{Parameters}

target Address of the symmetric data object where to store the results of the reduction operation.

source Address of the symmetric data object that contains the elements for each separate reduction operation.

nreduce The number of elements in the target and source arrays.

PE_start The lowest PE number of the active set of PEs.

logPE_stride The log (base 2) of the stride between consecutive PE numbers in the active set.

PE_size The number of PEs in the active set.

pWrk A symmetric work array. 
pSync A symmetric work array.

\section{Constraints}

- The target array on all PEs in the active set must be ready to accept the results of the reduction.

- target and source must be the addresses of symmetric data objects.

- If using $\mathrm{C} / \mathrm{C}++$, the type of target must match that implied in the Synopsis section. When calling from Fortran, the data type of target must be as follows:

- For SHMEM_INT4_SUM_TO_ALL(), target must be of type Integer, with element size of 4 bytes.

- For SHMEM_INT8_SUM_TO_ALL(), target must be of type Integer, with element size of 8 bytes.

- For SHMEM_REAL4_SUM_TO_ALL(), target must be of type Real, with element size of 4 bytes.

- For SHMEM_REAL8_SUM_TO_ALL(), target must be of type Real, with element size of 8 bytes.

- For SHMEM_REAL16_SUM_TO_ALL(), target must be of type Real, with element size of 16 bytes.

- source must be the same type as target.

- source and target may be the same array, but they must not be overlapping arrays.

- If using $\mathrm{C} / \mathrm{C}++$, nreduce must be of type integer. If you are using Fortran, it must be a default integer value.

- If using $\mathrm{C} / \mathrm{C}++$, PE_start must be of type integer. If you are using Fortran, it must be a default integer value. Its value must be greater than or equal to zero.

- If using $\mathrm{C} / \mathrm{C}++$, logPE_stride must be of type integer. If you are using Fortran, it must be a default integer value.

- If using $\mathrm{C} / \mathrm{C}++$, PE_size must be of type integer. If you are using Fortran, it must be a default integer value. Its value must be less than or equal to the total number of PEs minus one.

- The pWrk argument must have the same data type as target. In $\mathrm{C} / \mathrm{C}++$, this contains max(nreduce/2 + 1, _SHMEM_REDUCE_MIN_WRKDATA_SIZE) elements. In Fortran, this contains $\max (\mathbf{n r e d u c e} / 2+1$, SHMEM_REDUCE_MIN_WRKDATA_SIZE) elements. 
- In $\mathrm{C} / \mathrm{C}++$, pSync must be of type long and size _SHMEM_REDUCE_SYNC_SIZE. In Fortran, pSync must be of type integer and size SHMEM_REDUCE_SYNC_SIZE. If you are using Fortran, it must be a default integer value. Every element of this array must be initialized with the value _SHMEM_SYNC_VALUE (in C/C++) or SHMEM_SYNC_ VALUE (in Fortran) before any of the PEs in the active set enter the reduction routine.

- The pWrk and pSync arrays on all PEs in the active set must not be in use from a prior call to a collective OpenSHMEM routine.

- This routine must be called by all the PEs in the active set at the same point of the execution path; otherwise undefined behavior results.

\section{Effect}

The sum reduction routines compute the summation of nreduce elements in array source across all PEs in the active set. The active set of PEs is defined by the triple PE_start, logPE_stride and PE_size. The results of the reduction must be stored at address target on all PEs of the active set.

As with all OpenSHMEM collective routines, each of these routines assumes that only PEs in the active set call the routine. If a PE not in the active set calls a OpenSHMEM collective routine, undefined behavior results.

\section{Notes}

All OpenSHMEM reduction routines reset the values in pSync before they return, so a particular pSync buffer need only be initialized the first OpenSHMEM reduction routine. Be careful of the following situations: If the pSync array is initialized at run time, some type of synchronization is needed to ensure that all PEs in the working set have initialized pSync before any of them enter a OpenSHMEM routine called with the pSync synchronization array. A pSync or pWrk array can be reused in a subsequent reduction routine call only if none of the PEs in the active set are still processing a prior reduction routine call that used the same pSync or pWrk arrays. In general, this can be assured only by doing some type of synchronization. However, in the special case of reduction routines being called with the same active set, you can allocate two pSync and pWrk arrays and alternate between them on successive calls.

\section{Return Values}

Upon completion, the target array will be updated with the result of the operation and the elements of the pSync array will be restored to their initial values. 


\subsection{7 shmem_prod}

\section{Summary}

Computes the product of symmetric arrays over the active set of PEs.

\section{Synopsis}

$\mathrm{C} / \mathrm{C}++$ :

void shmem_short_prod_to_all(short *target, short $\star$ source, int nreduce, int

PE_start, int logPE_stride, int PE_size, int $\star$ pWrk, long $\star$ pSync);

void shmem_int_prod_to_all (int *target, int $\star$ source, int nreduce, int

PE_start, int logPE_stride, int PE_size, int $\star$ pWrk, long $\star$ pSync);

void shmem_long_prod_to_all(long *target, long $\star$ source, int nreduce, int

PE_start, int logPE_stride, int PE_size, long $\star$ pWrk, long $\star$ pSync);

void shmem_float_prod_to_all(float *target, float *source, int nreduce, int

PE_start, int logPE_stride, int PE_size, float $\star$ pWrk, long $\star$ pSync) ;

void shmem_double_prod_to_all(double $\star$ target, double $\star$ source, int nreduce,

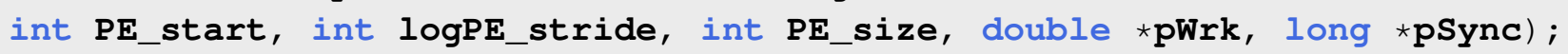

void shmem_longlong_prod_to_all(long long $\star$ target, long long $\star$ source, int

nreduce, int PE_start, int logPE_stride, int PE_size, long long $\star$ pWrk,

long $\star$ pSync);

void shmem_longdouble_prod_to_all(long double *target, long double $\star$ source,

int nreduce, int $\mathrm{PE}$ start, int $\log \mathrm{PE}$ stride, int $\mathrm{PE}$ size, long double

$\star$ pWrk, long $\star$ psync);

void shmem_complexf_prod_to_all(float complex *target, float complex

*source, int nreduce, int PE_start, int logPE_stride, int PE_size, float complex $\star$ pWrk, long $\star$ pSync);

void shmem_complexd_prod_to_all(double complex *target, double complex *source, int nreduce, int PE_start, int logPE_stride, int PE_size, double complex $\star$ pWrk, long $\star$ pSync);

Fortran:

INTEGER PSYNC (SHMEM_REDUCE_SYNC_SIZE)

INTEGER nreduce, PE_start, logPE_stride, PE_size

CALL SHMEM_INT4_PROD_TO_ALL(target, source, nreduce, PE_start, logPE_stride, PE_size, pWrk, pSync)

CALI SHMEM_INT8_PROD_TO_ALL (target, source, nreduce, PE_start, logPE_stride, PE_size, pWrk, pSync) 


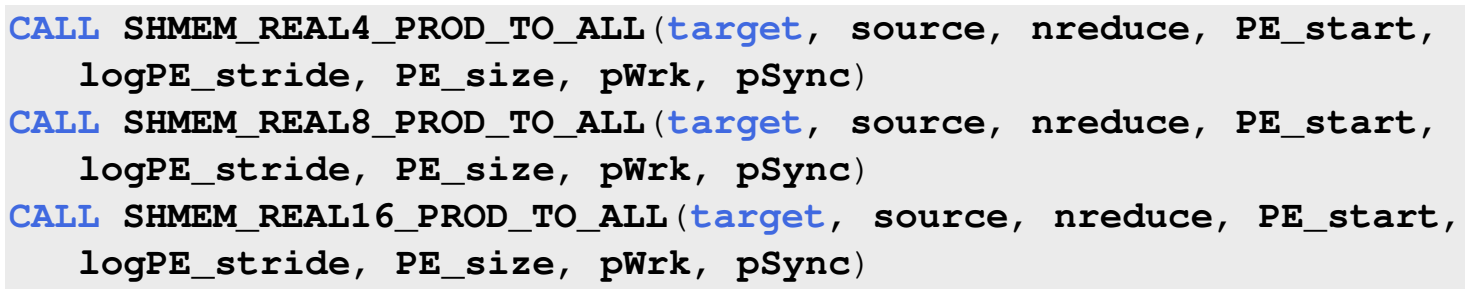

\section{Parameters}

target Address of the symmetric data object where to store the results of the reduction operation.

source Address of the symmetric data object that contains the elements for each separate reduction operation.

nreduce The number of elements in the target and source arrays.

PE_start The lowest PE number of the active set of PEs.

logPE_stride The log (base 2) of the stride between consecutive PE numbers in the active set.

PE_size The number of PEs in the active set.

pWrk A symmetric work array.

pSync A symmetric work array.

\section{Constraints}

- The target array on all PEs in the active set must be ready to accept the results of the reduction.

- target and source must be the addresses of symmetric data objects.

- If using $\mathrm{C} / \mathrm{C}++$, the type of target must match that implied in the Synopsis section. When calling from Fortran, the data type of target must be as follows:

- For SHMEM_INT4_PROD_TO_ALL(), target must be of type Integer, with element size of 4 bytes.

- For SHMEM_INT8_PROD_TO_ALL(), target must be of type Integer, with element size of 8 bytes. 
- For SHMEM_REAL4_PROD_TO_ALL(), target must be of type Real, with element size of 4 bytes.

- For SHMEM_REAL8_PROD_TO_ALL(), target must be of type Real, with element size of 8 bytes.

- For SHMEM_REAL16_PROD_TO_ALL(), target must be of type Real, with element size of 16 bytes.

- source must be the same type as target.

- source and target may be the same array, but they must not be overlapping arrays.

- If using $\mathrm{C} / \mathrm{C}++$, nreduce must be of type integer. If you are using Fortran, it must be a default integer value.

- If using $\mathrm{C} / \mathrm{C}++$, PE_start must be of type integer. If you are using Fortran, it must be a default integer value. Its value must be greater than or equal to zero.

- If using $\mathrm{C} / \mathrm{C}++$, logPE_stride must be of type integer. If you are using Fortran, it must be a default integer value.

- If using $\mathrm{C} / \mathrm{C}++$, PE_size must be of type integer. If you are using Fortran, it must be a default integer value. Its value must be less than or equal to the total number of PEs minus one.

- The pWrk argument must have the same data type as target. In $\mathrm{C} / \mathrm{C}++$, this contains max(nreduce/2 + 1, SHMEM_REDUCE_MIN_WRKDATA_SIZE) elements. In Fortran, this contains $\max (\mathbf{n} \bar{r} e d u c e / 2+1$, SHMEM_REDUCE_MIN_WRKDATA_SIZE) elements.

- In $\mathrm{C} / \mathrm{C}++$, pSync must be of type long and size _SHMEM_REDUCE_SYNC_SIZE. In Fortran, pSync must be of type integer and size SHMEM_REDUCE_SYNC_SIZEE. If you are using Fortran, it must be a default integer value. Every element of this array must be initialized with the value _SHMEM_SYNC_VALUE (in C/C++) or SHMEM_SYNC_ VALUE (in Fortran) before any of the PEs in the active set enter the reduction routine.

- The pWrk and pSync arrays on all PEs in the active set must not be in use from a prior call to a collective OpenSHMEM routine.

- This routine must be called by all the PEs in the active set at the same point of the execution path; otherwise undefined behavior results. 


\section{Effect}

The prod reduction routines compute the summation of nreduce elements in array source across all PEs in the active set. The active set of PEs is defined by the triple PE_start, logPE_stride and PE_size. The results of the reduction must be stored at address target on all PEs of the active set.

As with all OpenSHMEM collective routines, each of these routines assumes that only PEs in the active set call the routine. If a PE not in the active set calls a OpenSHMEM collective routine, undefined behavior results.

\section{Notes}

All OpenSHMEM reduction routines reset the values in pSync before they return, so a particular pSync buffer need only be initialized the first OpenSHMEM reduction routine. Be careful of the following situations: If the pSync array is initialized at run time, some type of synchronization is needed to ensure that all PEs in the working set have initialized pSync before any of them enter a OpenSHMEM routine called with the pSync synchronization array. A pSync or pWrk array can be reused in a subsequent reduction routine call only if none of the PEs in the active set are still processing a prior reduction routine call that used the same pSync or pWrk arrays. In general, this can be assured only by doing some type of synchronization. However, in the special case of reduction routines being called with the same active set, you can allocate two pSync and pWrk arrays and alternate between them on successive calls.

\section{Return Values}

Upon completion, the target array will be updated with the result of the operation and the elements of the pSync array will be restored to their initial values.

\subsection{Collect Routines}

\subsection{1 shmem_collect}

\section{Summary}

Concatenates blocks of data from multiple processing elements (PEs) to an array in every PE . 


\section{Synopsis}

$\mathrm{C} / \mathrm{C}++$ :

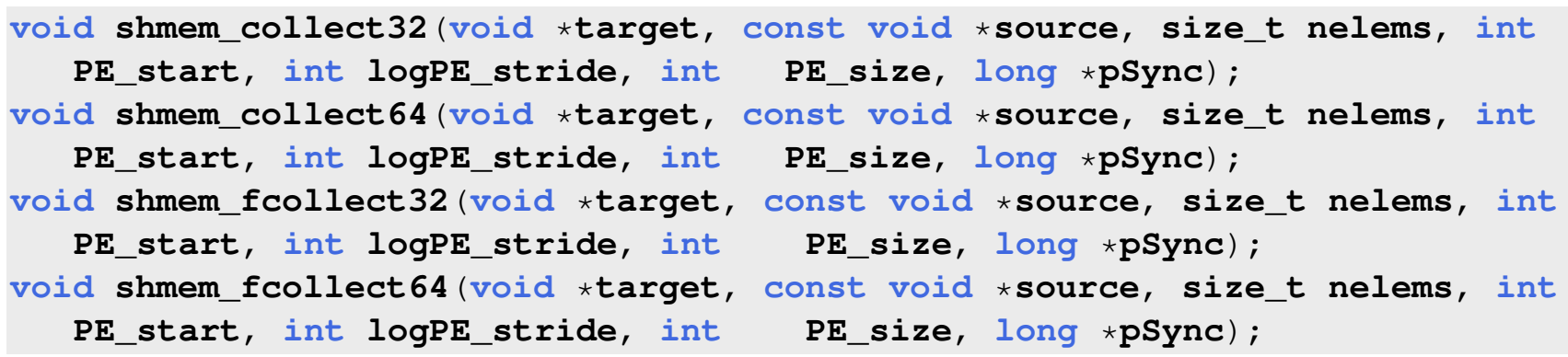

Fortran:

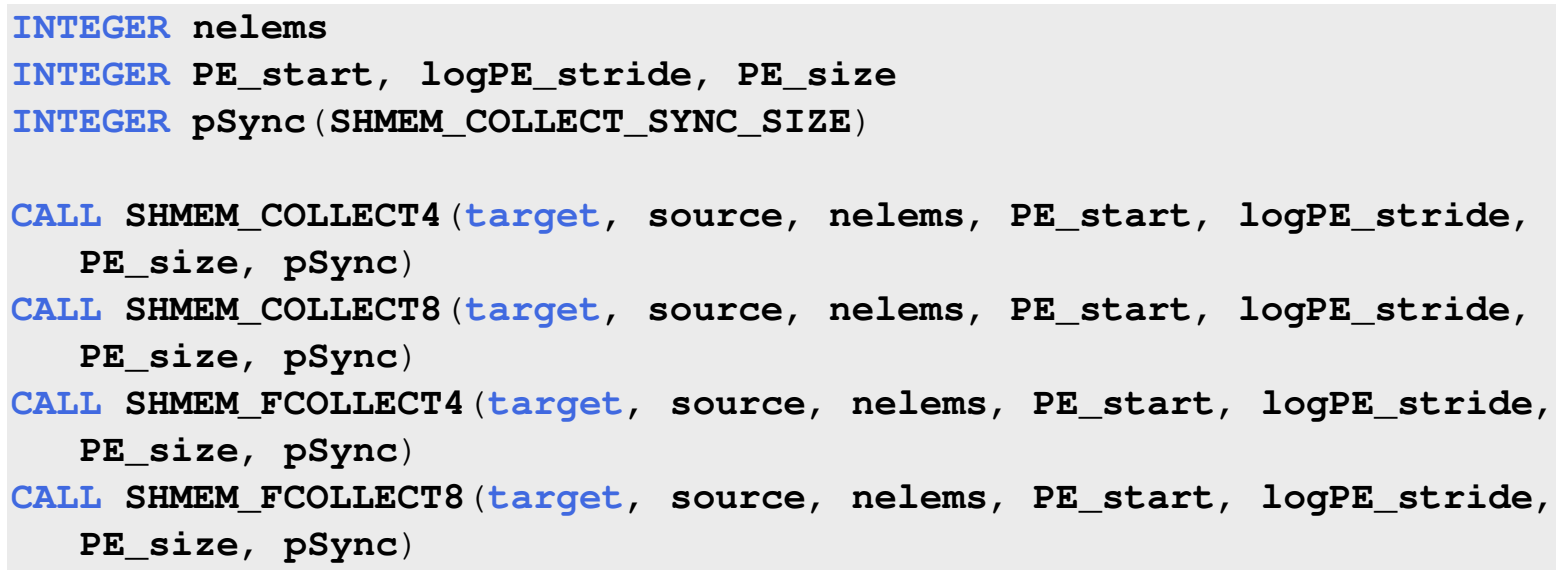

\section{Parameters}

target Address of the symmetric data object where to store the results of the collect operation.

source Address of the symmetric data object that contains the data to be concatenated.

nelems The number of elements in the source array.

PE_start The lowest PE number of the active set of PEs.

logPE_stride The log (base 2) of the stride between consecutive PE numbers in the active set.

PE_size The number of PEs in the active set.

pSync A symmetric work array. 


\section{Constraints}

- The target array on all PEs in the active set must be ready to accept the results of the concatenation. It must also be large enough to accept the results of the concatenation.

- target and source must be the addresses of symmetric data objects.

- Data types for target are as follows:

- For shmem_collect32(), shmem_fcollect32(), SHMEM_COLLECT4 and SHMEM_ FCOLLECT4, target must be of type Integer, with element size of 32 bytes.

- For shmem_collect64(), shmem_fcollect64(), SHMEM_COLLECT8 and SHMEM_ FCOLLECT8, target must be of type Integer, with element size of 64 bytes.

- source must be the same type as target.

- source and target may be the same array, but they must not be overlapping arrays.

- If using $\mathrm{C} / \mathrm{C}++$, nelems must be of type integer. If you are using Fortran, it must be a default integer value.

- If using $\mathrm{C} / \mathrm{C}++$, PE_start must be of type integer. If you are using Fortran, it must be a default integer value. Its value must be greater than or equal to zero.

- If using $\mathrm{C} / \mathrm{C}++$, logPE_stride must be of type integer. If you are using Fortran, it must be a default integer value.

- If using $\mathrm{C} / \mathrm{C}++$, PE_size must be of type integer. If you are using Fortran, it must be a default integer value. Its value must be less than or equal to the total number of PEs minus one.

- The pSync array on all PEs in the active set must not be in use from a prior call to a collective OpenSHMEM routine.

- This routine must be called by all the PEs in the active set at the same point of the execution path; otherwise undefined behavior results.

\section{Effect}

The collect routines concatenate nelems elements from array source across all PEs in the active set, and store the result in array target. The fcollect routines require that nelems be the same value in all participating PEs, while the collect routines allow nelems to vary from PE to PE.

As with all OpenSHMEM collective routines, each of these routines assumes that only PEs in the active set call the routine. If a PE not in the active set calls a OpenSHMEM collective routine, undefined behavior results. 


\section{Notes}

All OpenSHMEM reduction routines reset the values in pSync before they return, so a particular pSync buffer need only be initialized the first OpenSHMEM reduction routine. Be careful of the following situations: If the pSync array is initialized at run time, some type of synchronization is needed to ensure that all PEs in the working set have initialized pSync before any of them enter a OpenSHMEM routine called with the pSync synchronization array. A pSync or pWrk array can be reused in a subsequent reduction routine call only if none of the PEs in the active set are still processing a prior reduction routine call that used the same pSync or pWrk arrays. In general, this can be assured only by doing some type of synchronization. However, in the special case of reduction routines being called with the same active set, you can allocate two pSync and pWrk arrays and alternate between them on successive calls.

\section{Return Values}

Upon successful completion of these routines, target will have the result of the concatenation.

\subsection{Broadcast Routines}

\subsection{1 shmem_broadcast}

\section{Summary}

Copy a data object from a designated PE to a target location on all other PEs of the active set. See Figure 4 for a diagram of a simple broadcast operation. 


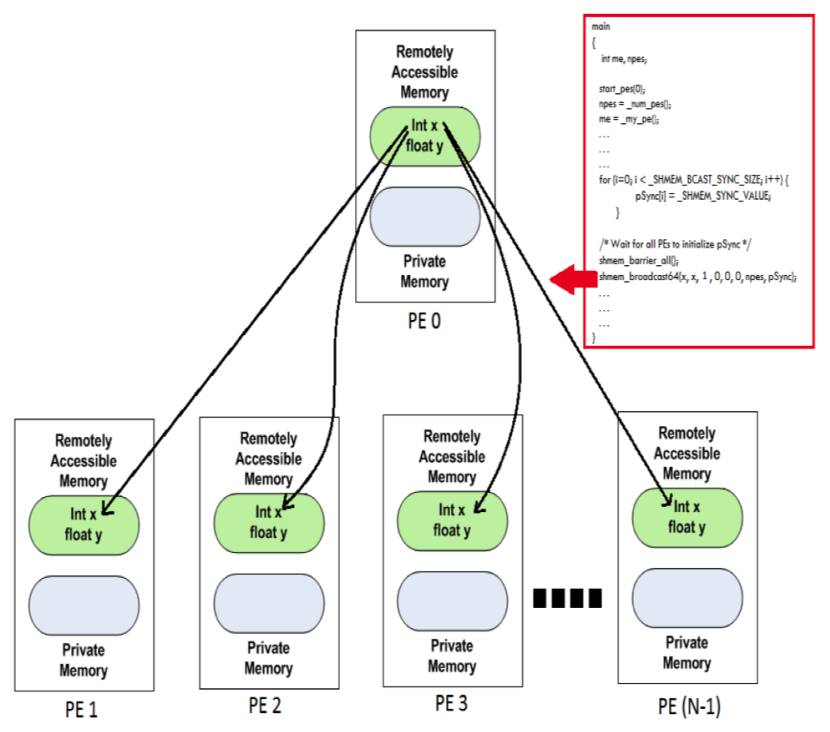

Fig. 4: Diagram of a simple broadcast operation.

\section{Synopsis}

$\mathrm{C} / \mathrm{C}++$ :

void shmem_broadcast32 (void *target, const void $\star$ source, size_t nelems, int PE_root, int PE_start, int logPE_stride, int PE_size, long $\star$ pSync) ; void shmem_broadcast64 (void *target, const void $\star$ source, size_t nelems, int PE_root, int $\mathrm{PE} \_s t a r t$, int $\log \mathrm{PE} \_s t r i d e$, int $\mathrm{PE}$ size, long $\star$ pSync);

\section{Fortran:}

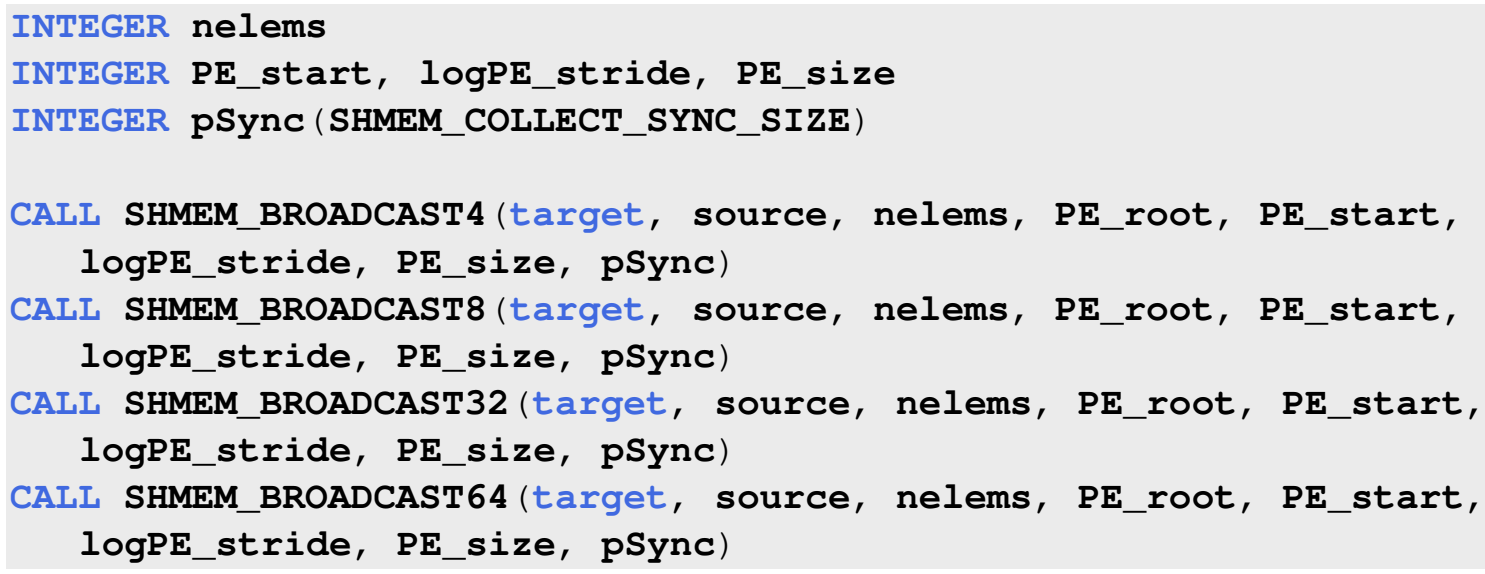




\section{Parameters}

target Address of the symmetric data object in which to store the data.

source Address of the symmetric data object that contains data to be copied.

nelems The number of elements in the target and source arrays.

PE_root The PE from which data will be copied.

PE_start The lowest PE number of the active set of PEs.

logPE_stride The log (base 2) of the stride between consecutive PE numbers in the active set.

PE_size The number of PEs in the active set.

pSync A symmetric work array.

\section{Constraints}

- The target array on all PEs in the active set must be ready to accept the results of the broadcast.

- source and target must be the addresses of symmetric data objects.

- Data types for target are as follows:

- For shmem_broadcast8() and shmem_broadcast64(), target may be of any non character type that has an element size of 64 bits. No Fortran derived types or $\mathrm{C} / \mathrm{C}++$ structures are allowed.

- For shmem_broadcast32(), target may be of any non character type that has an element size of 32 bits. No Fortran derived types or $\mathrm{C} / \mathrm{C}++$ structures are allowed.

- For shmem_broadcast4(), target may be of any non character type that has an element size of 32 bits.

- source must be the same type as target.

- source and target may be the same array, but they must not be overlapping arrays.

- If using $\mathrm{C} / \mathrm{C}++$, nelems must be of type integer. If you are using Fortran, it must be a default integer value.

- PE_root is a zero-based integer count into the active set: $0 \leq P E_{-}$root $<P E_{-}$size. If you are using Fortran, it must be a default integer value. 
- If using $\mathrm{C} / \mathrm{C}++$, PE_start must be of type integer. If you are using Fortran, it must be a default integer value. Its value must be greater than or equal to zero.

- If using $\mathrm{C} / \mathrm{C}++$, logPE_stride must be of type integer. If you are using Fortran, it must be a default integer value.

- If using $\mathrm{C} / \mathrm{C}++$, PE_size must be of type integer. If you are using Fortran, it must be a default integer value. Its value must be less than or equal to the total number of PEs minus one.

- In C/C++, pSync must be of type long and size _SHMEM_BCAST_SYNC_SIZE. In Fortran, pSync must be of type integer and size SHMEM_BCAST_SYNC_SIZE. Every element of this array must be initialized with the value _SHMEM_SYNC_VALUE (in $\mathrm{C} / \mathrm{C}++$ ) or SHMEM_SYNC_VALUE (in Fortran) before any $\overline{-}^{-}$the PEs in the active set enter the broadcast.

- The pSync array on all PEs in the active set must not be in use from a prior call to a collective OpenSHMEM routine.

- This routine must be called by all the PEs in the active set at the same point of the execution path; otherwise undefined behavior results.

\section{Effect}

The broadcast routines write the data at address source of the PE specified by PE_root to address target on all other PEs in the active set. The active set of PEs is defined by the triple PE_start, logPE_stride and PE_size. The data is not copied to the target address on the PE specified by PE_root.

Before returning, the broadcast routines ensure that the elements of the pSync array are restored to their initial values.

As with all OpenSHMEM collective routines, each of these routines assumes that only PEs in the active set call the routine. If a PE not in the active set calls a OpenSHMEM collective routine, undefined behavior results.

\section{Notes}

You must ensure that the pSync array is not being updated by any PE in the active set while any of the PEs participates in processing of an OpenSHMEM broadcast routine.

Be careful to avoid these situations: If the pSync array is initialized at run time, some type of synchronization is needed to ensure that all PEs in the working set have initialized pSync 
before any of them enter an OpenSHMEM routine called with the pSync synchronization array.

A pSync array may be reused on a subsequent OpenSHMEM broadcast routine only if none of the PEs in the active set are still processing a prior OpenSHMEM broadcast routine call that used the same pSync array. In general, this can be ensured only by doing some type of synchronization. However, in the special case of OpenSHMEM routines being called with the same active set, you can allocate two pSync arrays and alternate between them on successive calls.

\section{Return Values}

None.

\subsection{Lock Routines}

The OpenSHMEM lock routines manage mutual exclusion memory locks. These routines are appropriate for protecting a critical region from simultaneous update by multiple PEs.

\subsection{1 shmem_set_lock}

\section{Summary}

Sets a mutual exclusion memory lock after it is no longer in use by another process.

\section{Synopsis}

$\mathrm{C} / \mathrm{C}++$ :

void shmem_set_lock(long $\star$ lock);

Fortran:

INTEGER lock

CALI SHMEM_SET_LOCK (lock)

\section{Parameters}

lock Address of a symmetric data object that is a scalar variable or an array of length 1 . 


\section{Constraints}

- The value at address lock must be set to 0 on all PEs prior to the first use.

- If using $\mathrm{C} / \mathrm{C}++$, lock must be of type integer. If you are using Fortran, it must be of default kind.

\section{Effect}

The shmem_set_lock() routine sets a mutual exclusion lock after waiting for the lock to be freed by any other PE currently holding the lock. Waiting PEs are assured of getting the lock in a first-come, first-served manner.

\section{Return Values}

None.

\subsection{2 shmem_clear_lock}

\section{Summary}

Releases a lock previously set by the calling PE.

\section{Synopsis}

$\mathrm{C} / \mathrm{C}++$ :

void shmem_clear_lock (long $\star l o c k) ;$

Fortran:

INTEGER lock

CALI SHMEM_CLEAR_LOCK (lock)

\section{Parameters}

lock Address of a symmetric data object that is a scalar variable or an array of length 1 . 


\section{Constraints}

- If using $\mathrm{C} / \mathrm{C}++$, lock must be of type integer. If you are using Fortran, it must be of default kind.

- The lock can only be released by the PE that previously set the lock.

\section{Effect}

The shmem_clear_lock() routine releases a lock previously set by shmem_set_lock() after ensuring that all local and remote stores initiated in the critical region are complete.

\section{Return Values}

None.

\subsection{3 shmem_test_lock}

\section{Summary}

Sets a mutual exclusion lock only if it is currently cleared.

\section{Synopsis}

$\mathrm{C} / \mathrm{C}++$ :

int shmem_test_lock(long $\star$ lock);

Fortran:

INTEGER lOCk, SHMEM_TEST_LOCK

$I$ = SHMEM_TEST_LOCK ( lock)

\section{Parameters}

lock Address of a symmetric data object that is a scalar variable or an array of length 1.

\section{Constraints}

- If using $\mathrm{C} / \mathrm{C}++$, lock must be of type integer. If you are using Fortran, it must be of default kind. 


\section{Effect}

The shmem_test_lock() function sets a mutual exclusion lock only if it is currently cleared. By using this function, a PE can avoid blocking on a set lock. If the lock is currently set, the function returns without waiting.

\section{Return Values}

0 The lock was originally cleared and this call was able to set the lock.

1 The lock had been set and the call returned without waiting to set the lock.

Note to implementers: the lock variable is initialized to 0 everywhere. Once set, the value of the lock should be treated as opaque to allow implementations freedom to optimize lock structures, e.g. for specific hardware operations. 


\section{Library Constants}

\subsection{Constants Related To Reduction Operations}

\subsubsection{SHMEM_BCAST_SYNC_SIZE, _SHMEM_BCAST_SYNC_SIZE}

Length of the pSync arrays needed for broadcast operations. The value of this constant is implementation specific. Refer to the Broadcast Routines section under Library Routines for more information about the usage of this constant.

\subsubsection{SHMEM_SYNC_VALUE, _SHMEM_SYNC_VALUE}

Holds the value used to initialize the elements of pSync arrays. The value of this constant is implementation specific.

\subsubsection{SHMEM_REDUCE_SYNC_SIZE, _SHMEM_REDUCE_SYNC_SIZE}

Length of the work arrays needed for reduction operations. The value of this constant is implementation specific. Refer to the Reduction Routines section under Library Routines for more information about the usage of this constant.

\subsubsection{SHMEM_BARRIER_SYNC_SIZE, _SHMEM_BARRIER_SYNC_SIZE}

Length of the work array needed for barrier operations. The value of this constant is implementation specific. Refer to the Barrier Synchronization Routines section under Library Routines for more information about the usage of this constant.

\subsubsection{SHMEM_COLLECT_SYNC_SIZE,_SHMEM_COLLECT_SYNC_SIZE}

Length of the work array needed for collect operations. The value of this constant is implementation specific. Refer to the Collect Routines section under Library Routines for more information about the usage of this constant.

\subsubsection{SHMEM_REDUCE_MIN_WRKDATA_SIZE, _SHMEM_REDUCE_MIN_WRKDATA_ SIZE}

Minimum length of work arrays used in various collective operations. 


\section{Writing OpenSHMEM Programs}

\subsection{Incorporating OpenSHMEM into Programs}

$\mathrm{C}$ and $\mathrm{C}++$ programs that use the OpenSHMEM library must

\#include <shmem.h>

All Fortran OpenSHMEM programs should

include 'shmem. fh'

and Fortran OpenSHMEM programs that use constants defined by OpenSHMEM must

include 'shmem.fh'

\subsubsection{Compatibility Note}

Implementations must also provide these header files so that they can be referenced in C and $\mathrm{C}++$ as

\#include <mpp/shmem.h>

and in Fortran as

include 'mpp/shmem. $f$ h'

for backward compatbility with OpensHMEM 1.0 and SGI SHMEM.

\subsection{Initialization}

An OpenSHMEM program must call start_pes() (See Section Subsection 8.1.1) before any other OpenSHMEM routine. If start_pes() is not called first, the subsequent behavior of OpenSHMEM is undefined. Calling start_pes() more than once has no subsequent effect. The parameter to start_pes() is ignored; the number of PEs is taken from the invoking environment. 


\section{A Deprecated Items}

All of the items (e.g. subroutines, constants) in this section are present in OpenSHMEM purely for backwards compatibility with some older versions of SHMEM that pre-date OpenSHMEM, and should be avoided in new programs. Implementations must include all items in this section, but the subroutines below must have no effect ("no-ops"), conform to this API, and be documented as such.

\section{A.1 Cache Management Routines}

\section{A.1.1 shmem_set_cache_inv (deprecated)}

\section{Summary}

Enables automatic cache coherency mode.

\section{Synopsis}

$\mathrm{C} / \mathrm{C}++$ :

void shmem_set_cache_inv(void);

Fortran:

CALI SHMEM_SET_CACHE_INV ()

\section{Parameters}

None.

\section{Constraints}

None.

Effect

None.

\section{Return Values}

None. 


\section{A.1.2 shmem_set_cache_line_inv (deprecated)}

\section{Summary}

Enable cache coherency for a specific object only.

\section{Synopsis}

$\mathrm{C} / \mathrm{C}++$ : void shmem_set_cache_line_inv(void *target);

Fortran:

CALI SHMEM_SET_CACHE_LINE_INV(target)

\section{Parameters}

target Address of the symmetric data object.

\section{Constraints}

- If using $\mathrm{C} / \mathrm{C}++$, target can be of any non character type. If you are using Fortran, it can be of any kind.

\section{Effect}

None.

\section{Return Values}

None.

\section{A.1.3 shmem_clear_cache_inv (deprecated)}

\section{Summary}

Disable cache coherency. 


\section{Synopsis}

$\mathrm{C} / \mathrm{C}++$ :

void shmem_clear_cache_inv(void);

Fortran:

CALI SHMEM_CLEAR_CACHE_INV ( )

\section{Parameters}

None.

\section{Constraints}

None.

Effect

None.

\section{Return Values}

None.

A.1.4 shmem_clear_cache_line_inv (deprecated)

\section{Summary}

Disable cache coherency.

\section{Synopsis}

$\mathrm{C} / \mathrm{C}++$ :

void shmem_clear_cache_line_inv(void* target);

Fortran:

CALI SHMEM_CLEAR_CACHE_LINE_INV (target) 
A DEPRECATED ITEMS

Parameters

target Address of the symmetric data object.

\section{Constraints}

None.

Effect

None.

\section{Return Values}

None.

\section{A.1.5 shmem_udcflush (deprecated)}

\section{Summary}

Makes the entire user data cache coherent.

\section{Synopsis}

$\mathrm{C} / \mathrm{C}++$ :

void shmem_udcflush (void) ;

Fortran:

CALL SHMEM_UDCFLUSH ( )

\section{Parameters}

None.

\section{Constraints}

None. 


\section{Effect}

None.

\section{Return Values}

None.

\section{A.1.6 shmem_udcflush_line (deprecated)}

\section{Summary}

Enable cache coherency for a specified data object only.

\section{Synopsis}

$\mathrm{C} / \mathrm{C}++$ :

void shmem_udcflush_line (void *target);

Fortran:

CALI SHMEM_UDCFLUSH_LINE (target)

\section{Parameters}

target Address of the symmetric data object.

\section{Constraints}

- If using $\mathrm{C} / \mathrm{C}++$, target can be of any non character type. If you are using Fortran, it can be of any kind.

Effect

None.

\section{Return Values}

None. 


\section{B Environment Variables}

Consistent with the SGI implementation of SHMEM, the OpenSHMEM specification currently provides a set of environment variables that allows users to affect run-time behavior:

\begin{tabular}{|l|l|l|}
\hline Variable & Value & Function \\
\hline \hline SMA_VERSION & any & print the library version at start-up \\
\hline SMA_INFO & any & print helpful text about all these environment variable \\
\hline SMA_SYMMETRIC_SIZE & non-negative integer & number of bytes to allocate for symmetric heap \\
\hline SMA_DEBUG & any & enable debugging messages \\
\hline
\end{tabular}

Implementations are free to define their own environment variables. 


\section{Compiling and Running Applications}

The OpenSHMEM specification is silent regarding how OpenSHMEM programs are compiled, linked and run. This section shows some examples of how wrapper programs could be utilized to compile and launch applications. The commands are styled after wrapper programs found in many MPI implementations.

\section{C.1 Compilation}

\section{C.1.1 Applications written in C}

Assuming that the implementation provides a wrapper program named oshcc, to aid in the compilation of $\mathrm{C}$ applications, the wrapper could be called as follows:

oshcc <compiler options> -o myprogram myprogram.c

The program arguments for oshcc are:

<compiler options> Options understood by the underlying C compiler called by oshcc.

\section{C.2 Applications written in $\mathrm{C}++$}

Assuming that the implementation provides a wrapper program named oshCC, to aid in the compilation of $\mathrm{C}++$ applications, the wrapper could be called as follows:

oshCC <compiler options> -o myprogram myprogram.cpp

The program arguments for oshCC are:

<compiler options> Options understood by the underlying $\mathrm{C}++$ compiler called by oshCC.

\section{C.3 Applications written in Fortran}

Assuming that the implementation provides a wrapper program named oshfort, to aid in the compilation of Fortran applications, the wrapper could be called as follows:

oshfort <compiler options> -o myprogram myprogram.f

The program arguments for oshfort are:

<compiler options> Options understood by the underlying Fortran compiler called by oshfort. 


\section{Running Applications}

Assuming that the implementation provides a wrapper program named oshrun, to launch OpenSHMEM applications, the wrapper could be called as follows:

oshrun <additional options $>-\mathbf{n p}<\#><$ program $>$ <program arguments $>$

The program arguments for oshrun are:

<additional options> options passed to the underlying launcher

$-n p<\#>$ The number of processing elements (PEs) to be used in the execution.

$<$ program $>$ The program executable to be launched

$<$ program arguments $>$ flags and other parameters to pass to the program 
E EXAMPLES

E Examples

1.0 FINAL 


\section{E.1 C Language Examples}

Listing 1: Program that is a trivial Hello World.

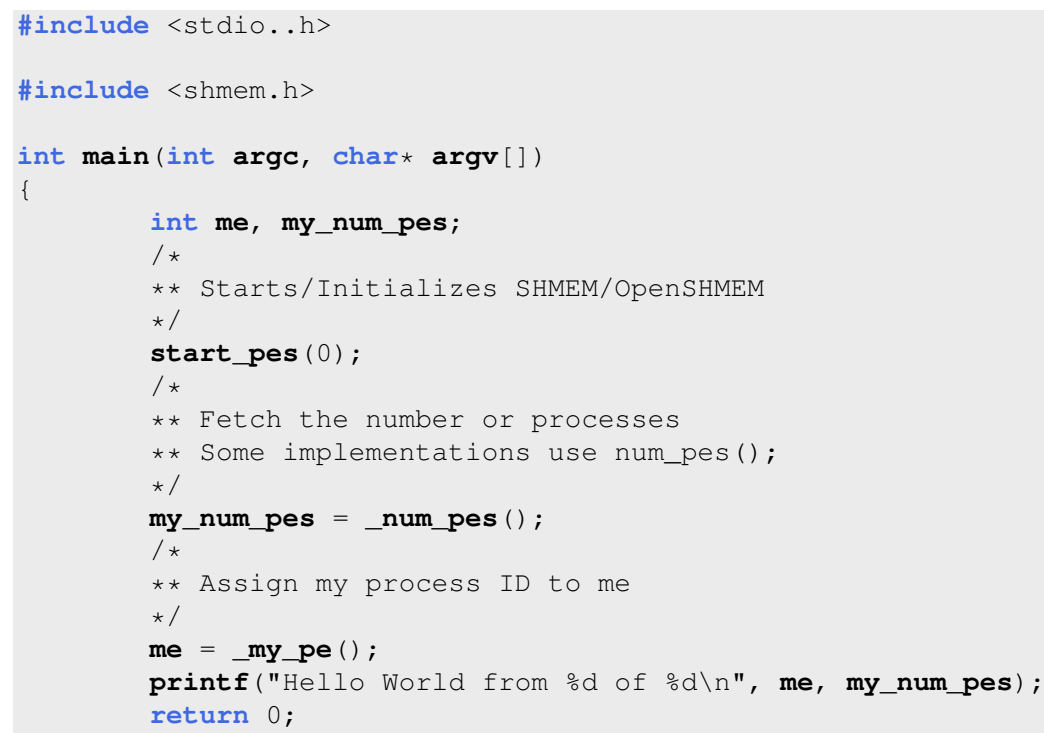

Listing 2: Program that implements a Circular Shift.

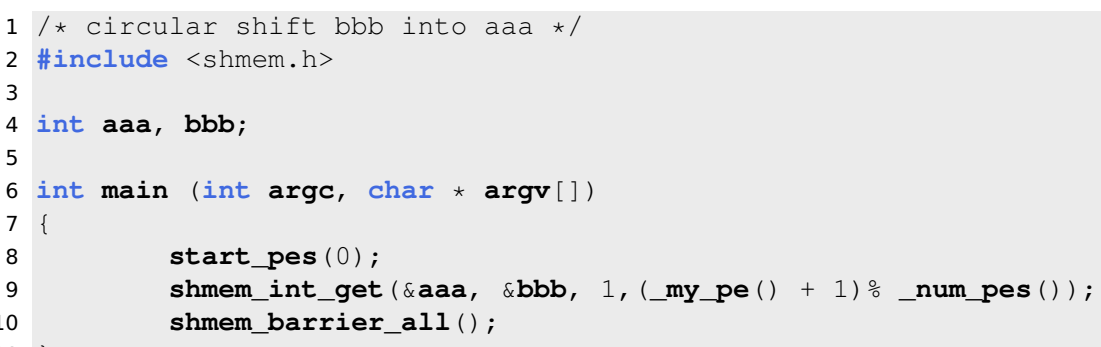


Listing 3: Program that demonstrates the use of shmalloc.

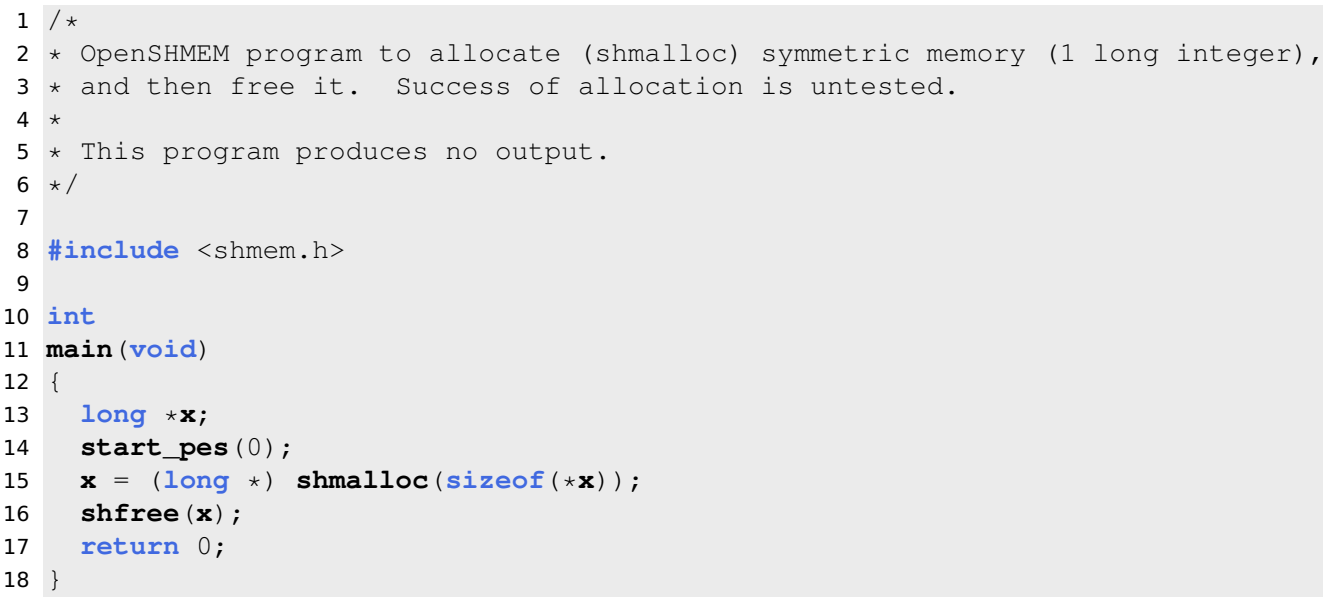

Listing 4: Program that implements Ping.

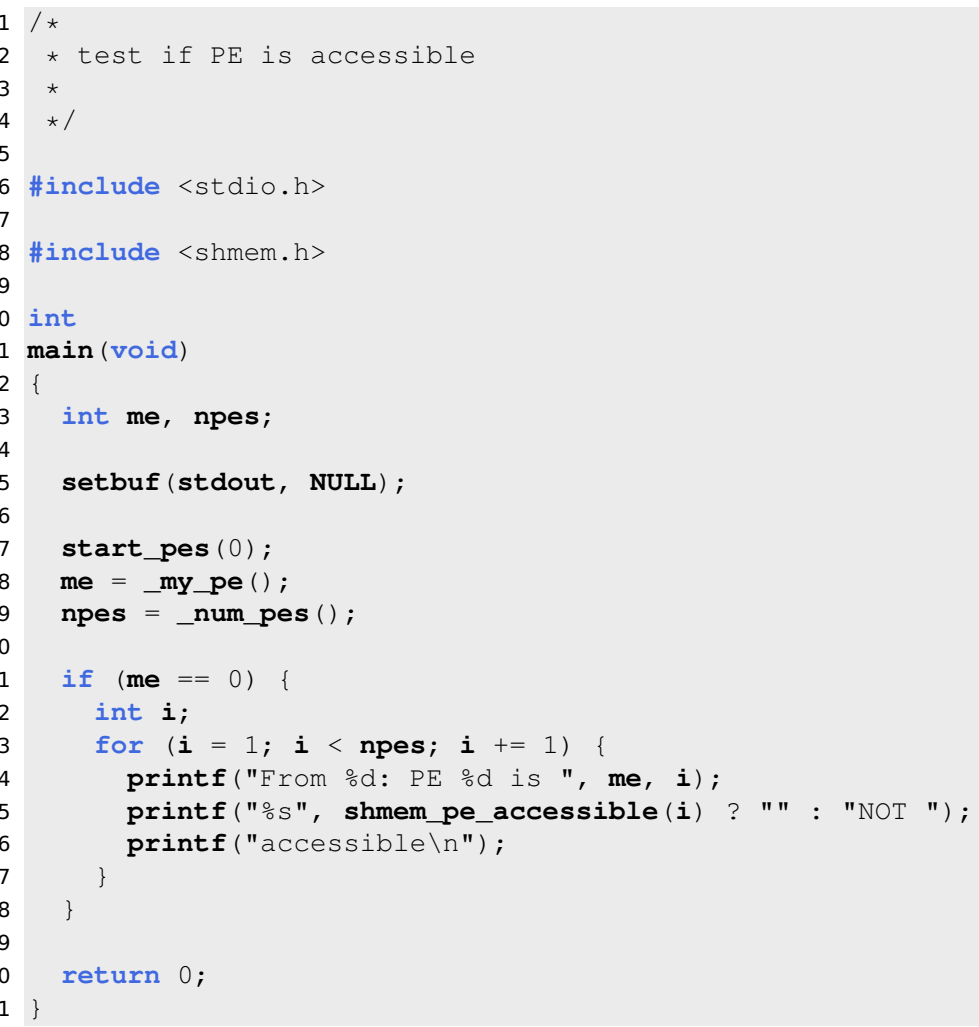


Listing 5: Program that uses the MAX reduction.

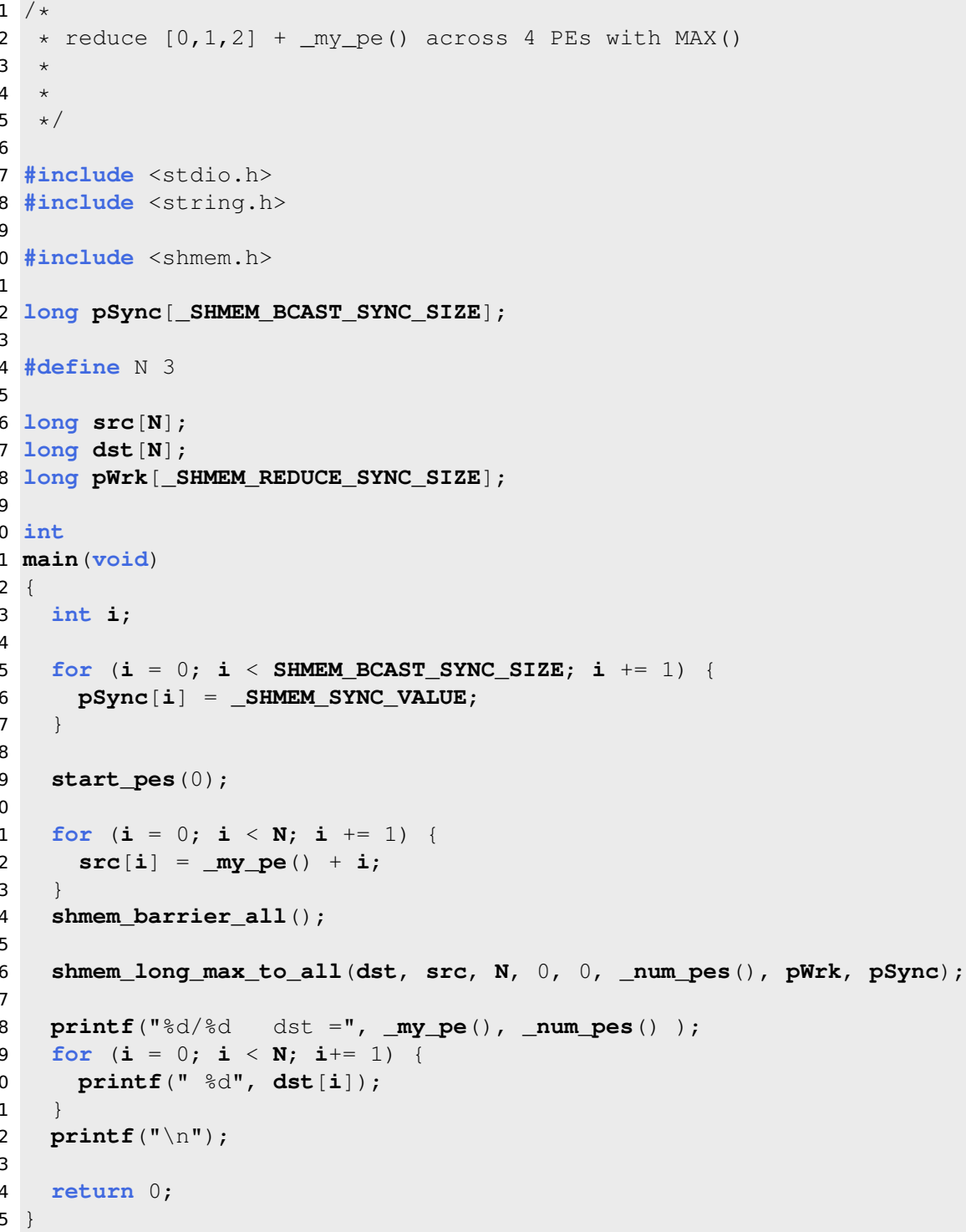




\section{Listing 6: Program that makes use of strided puts.}

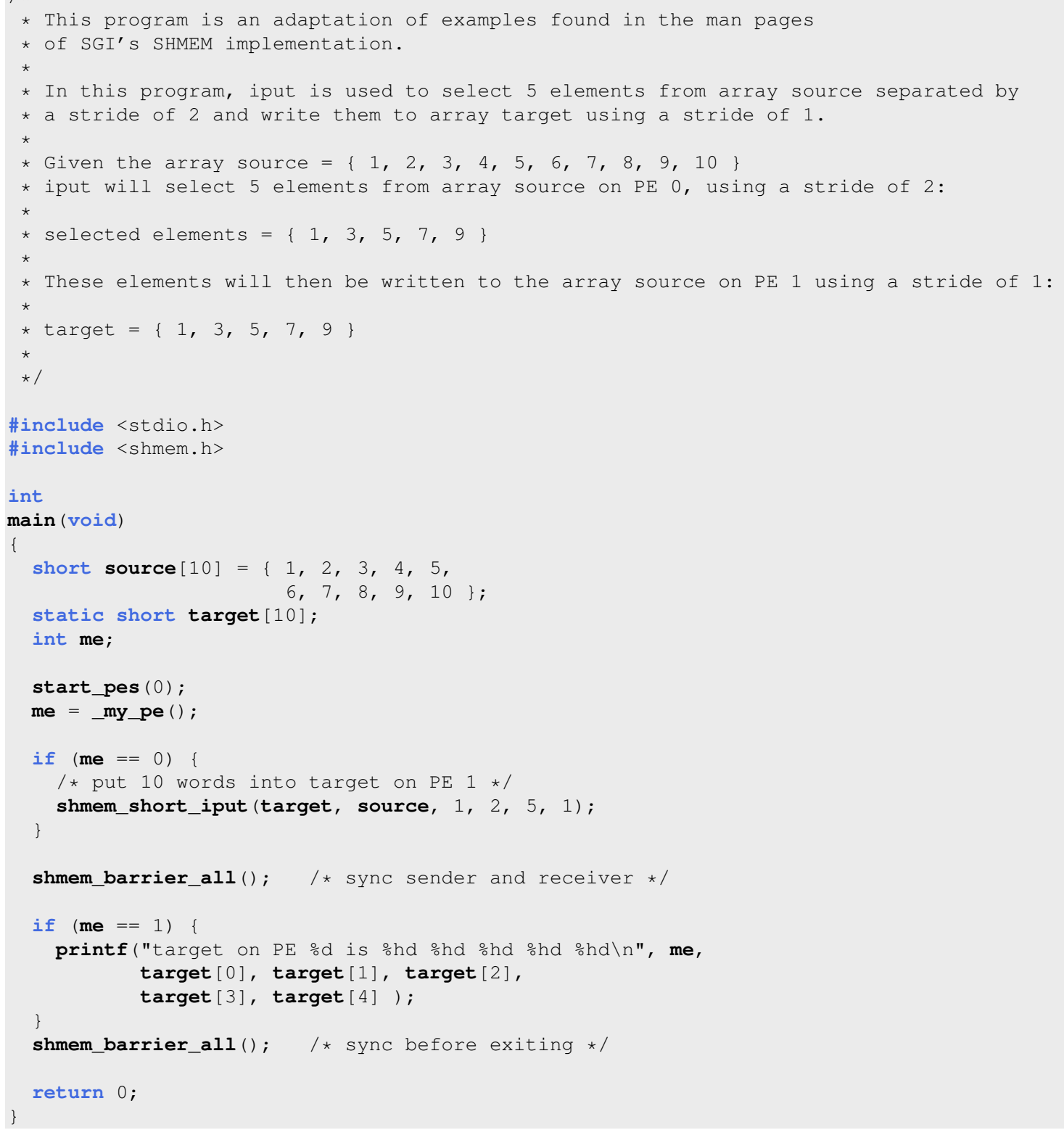


Listing 7: Program that implements an ALL-2-ALL (header)

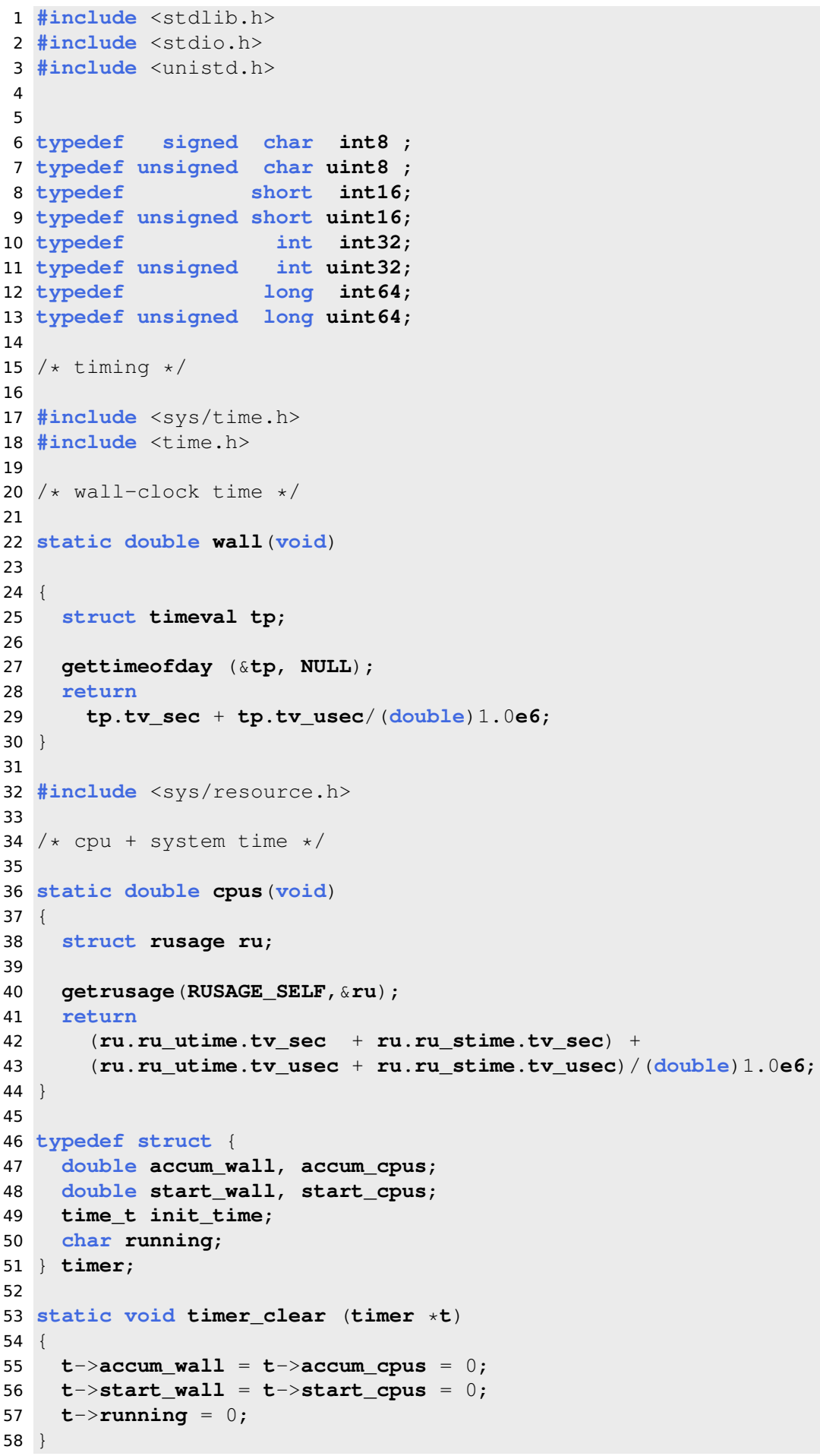




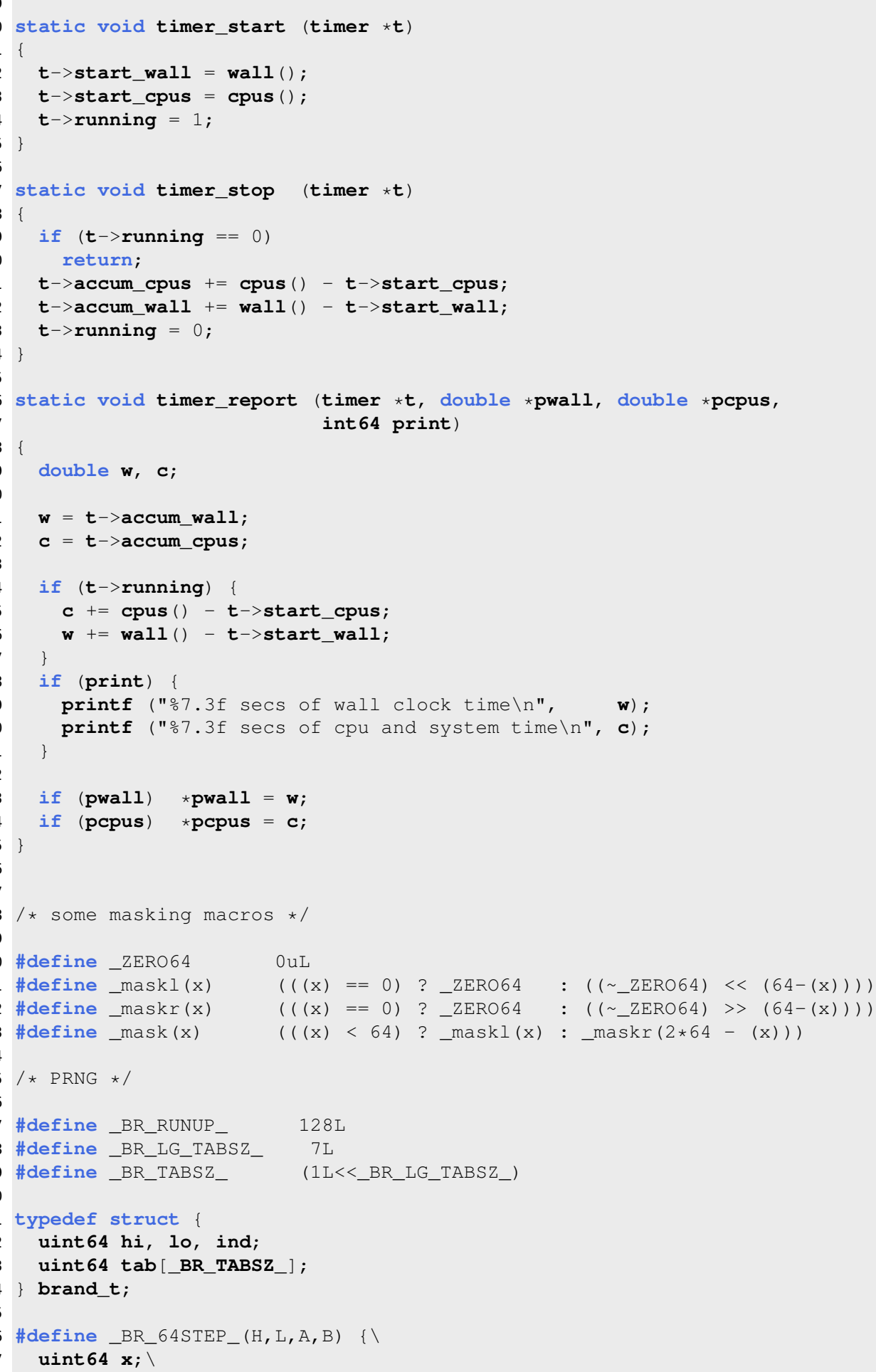




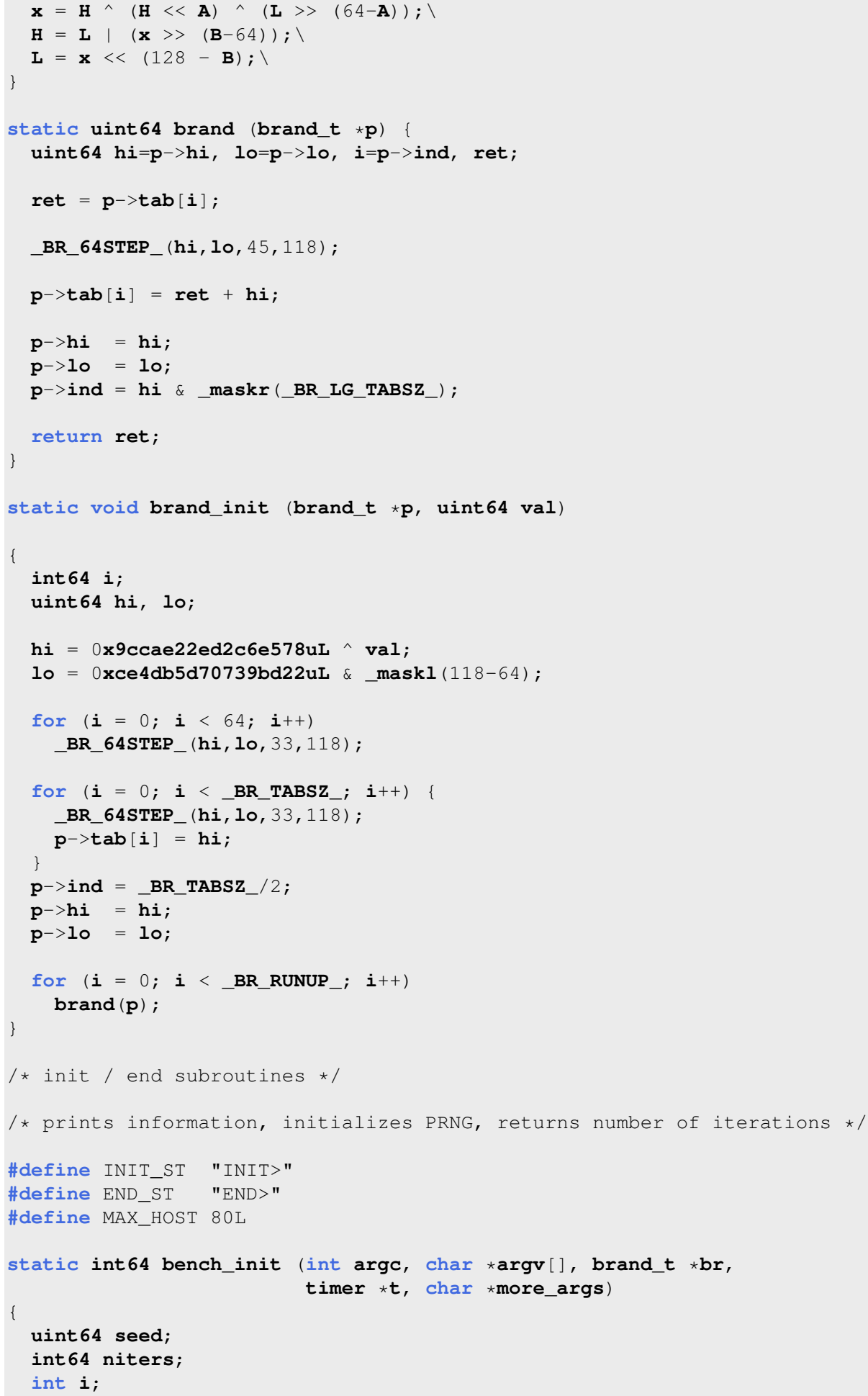




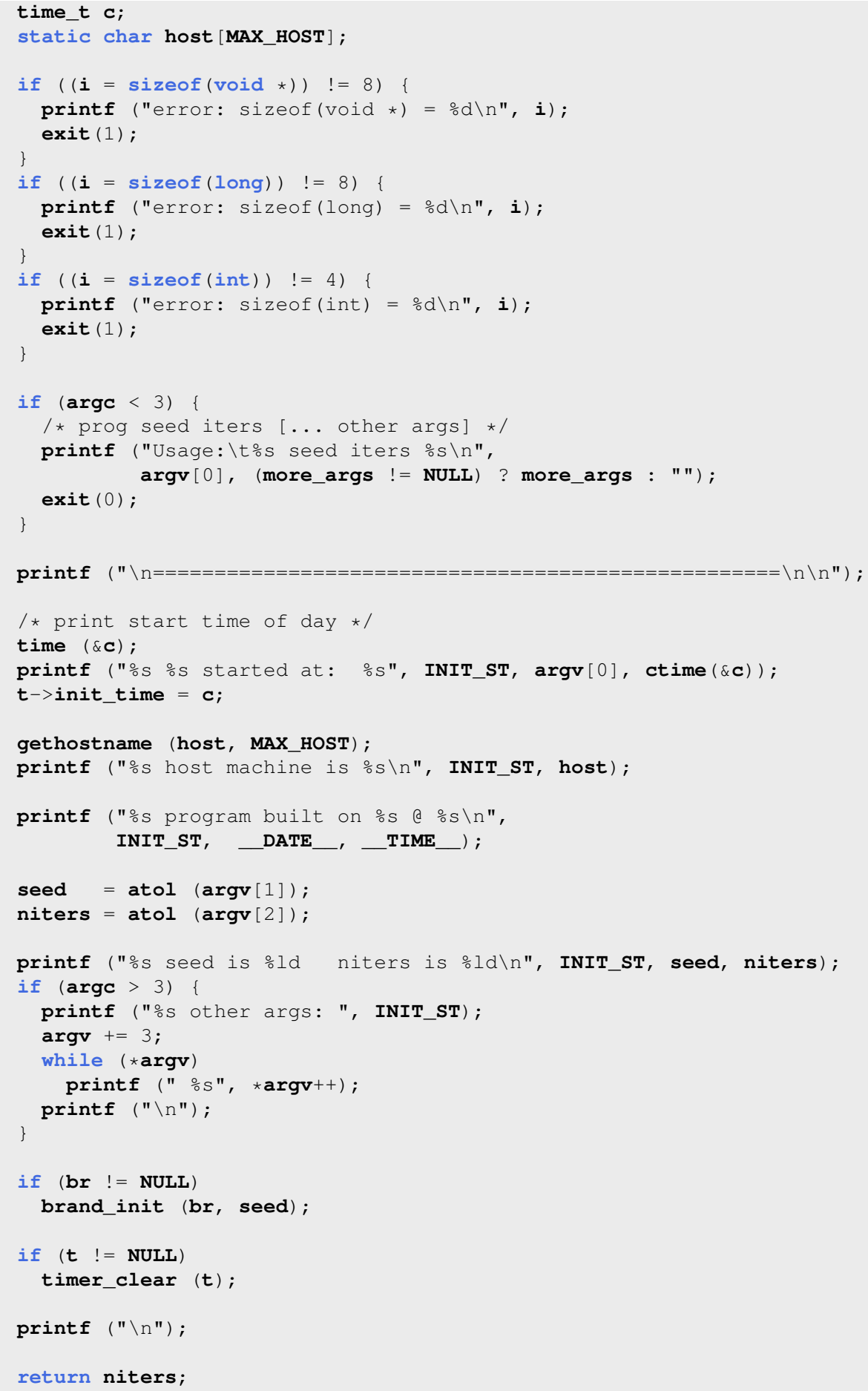




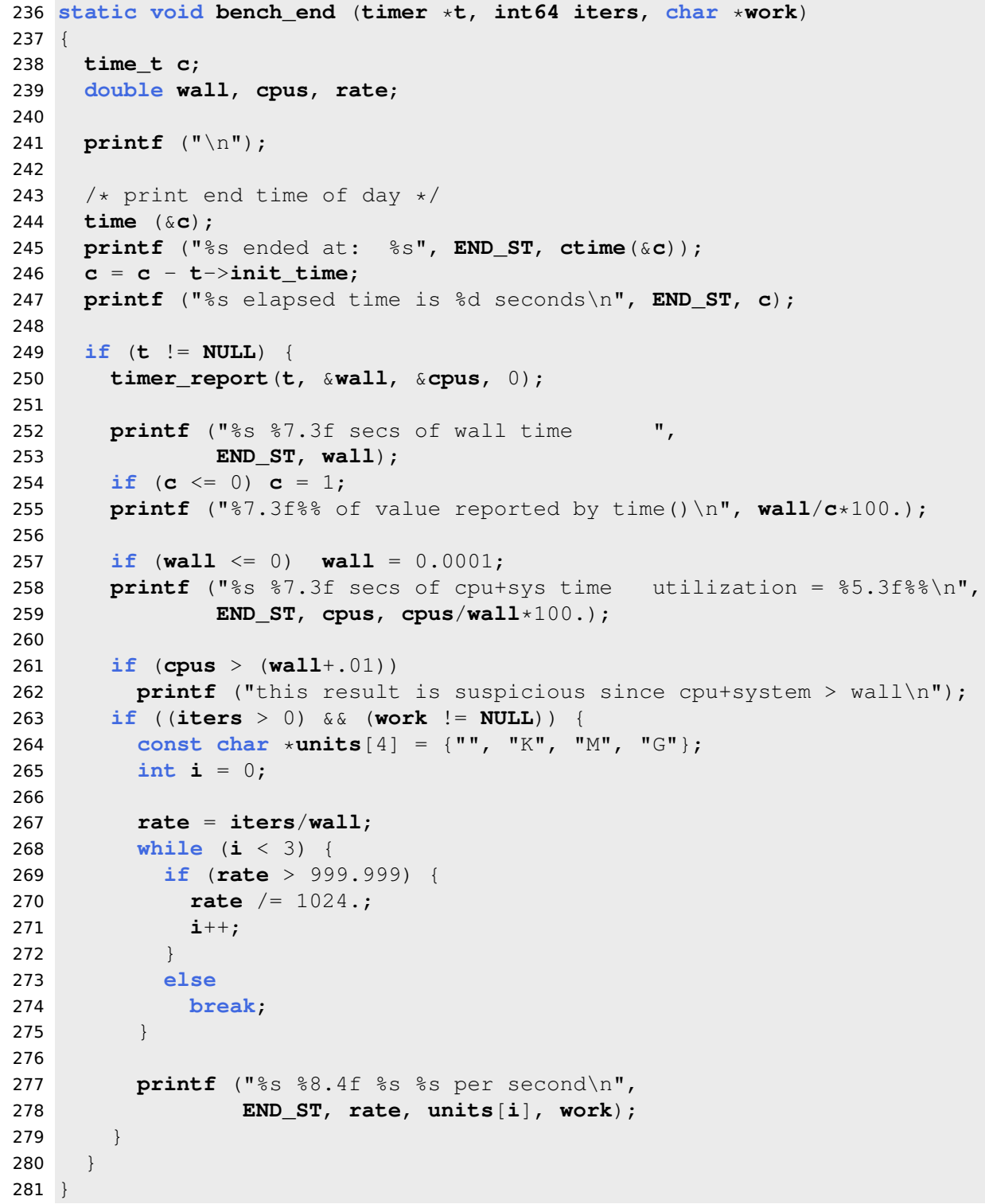


Listing 8: Program that implements an ALL-2-ALL (main)

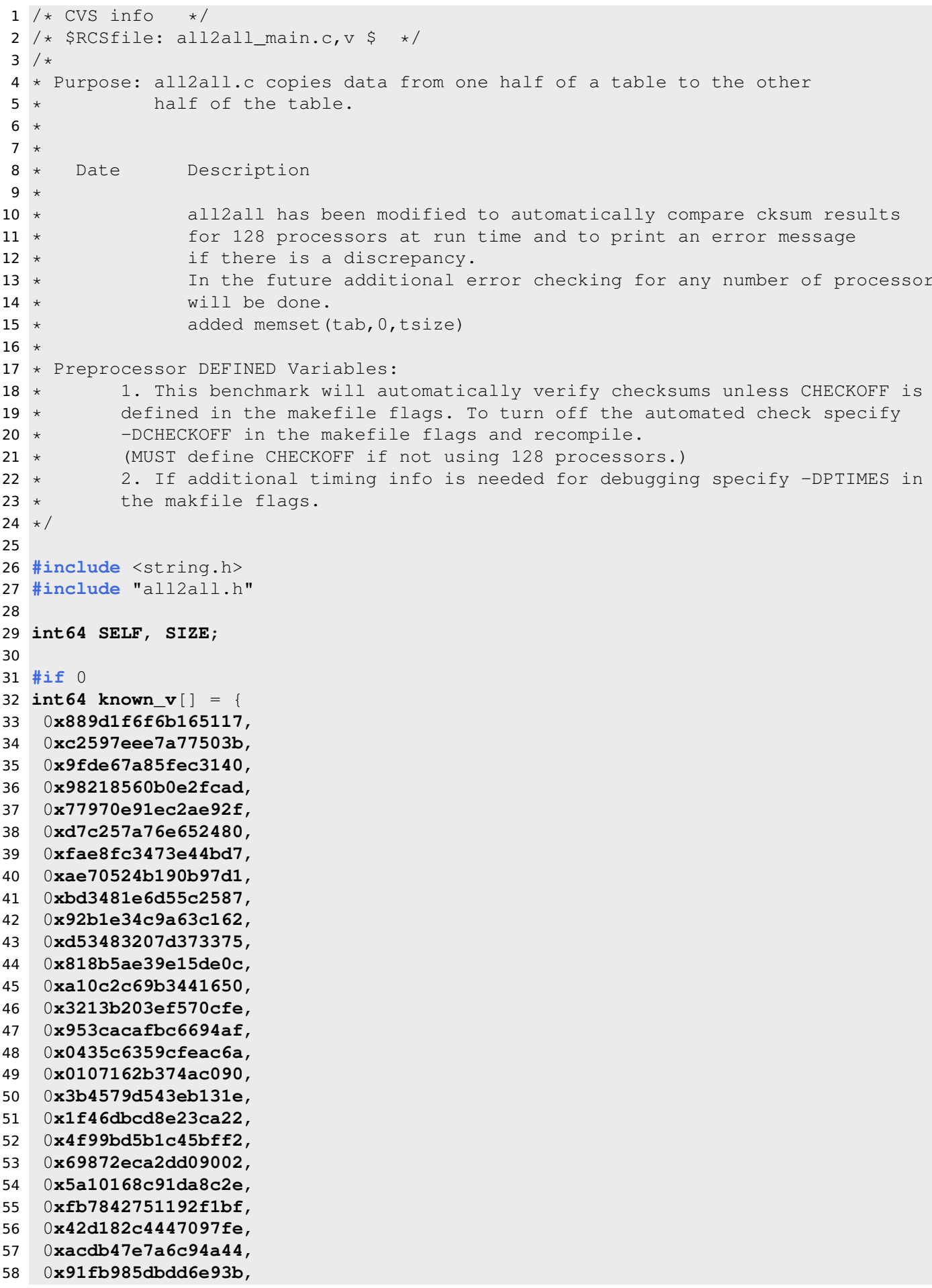




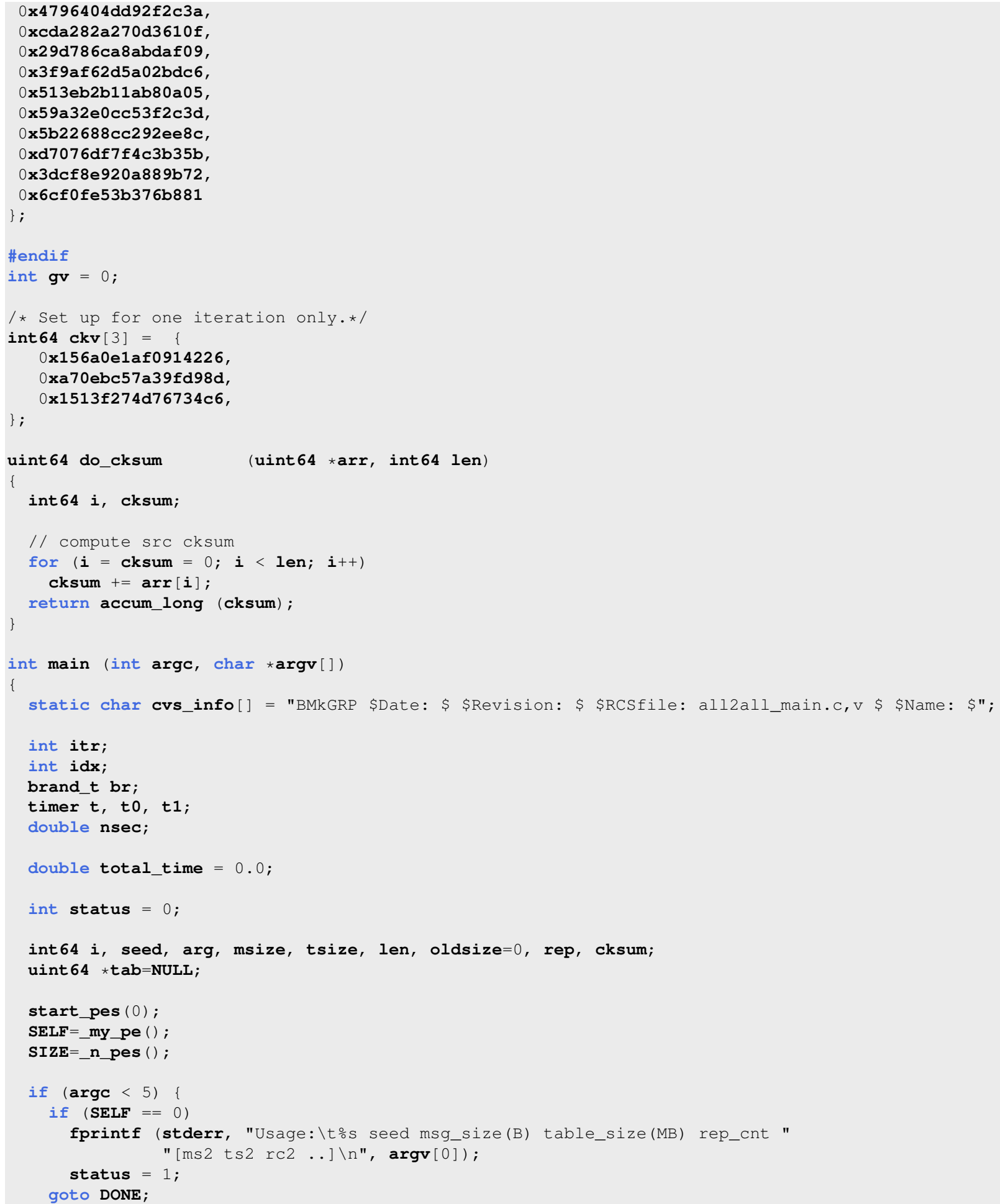




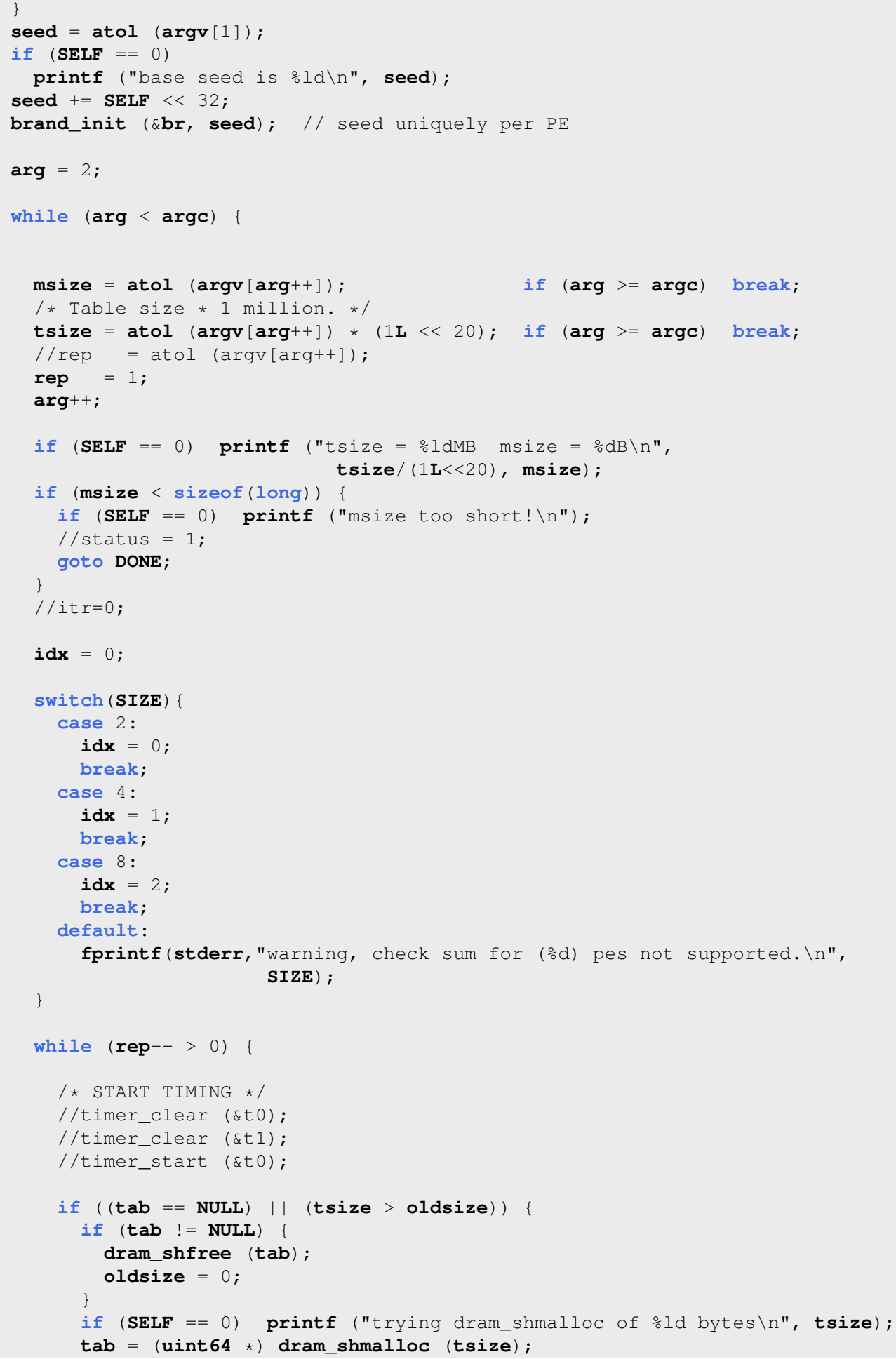




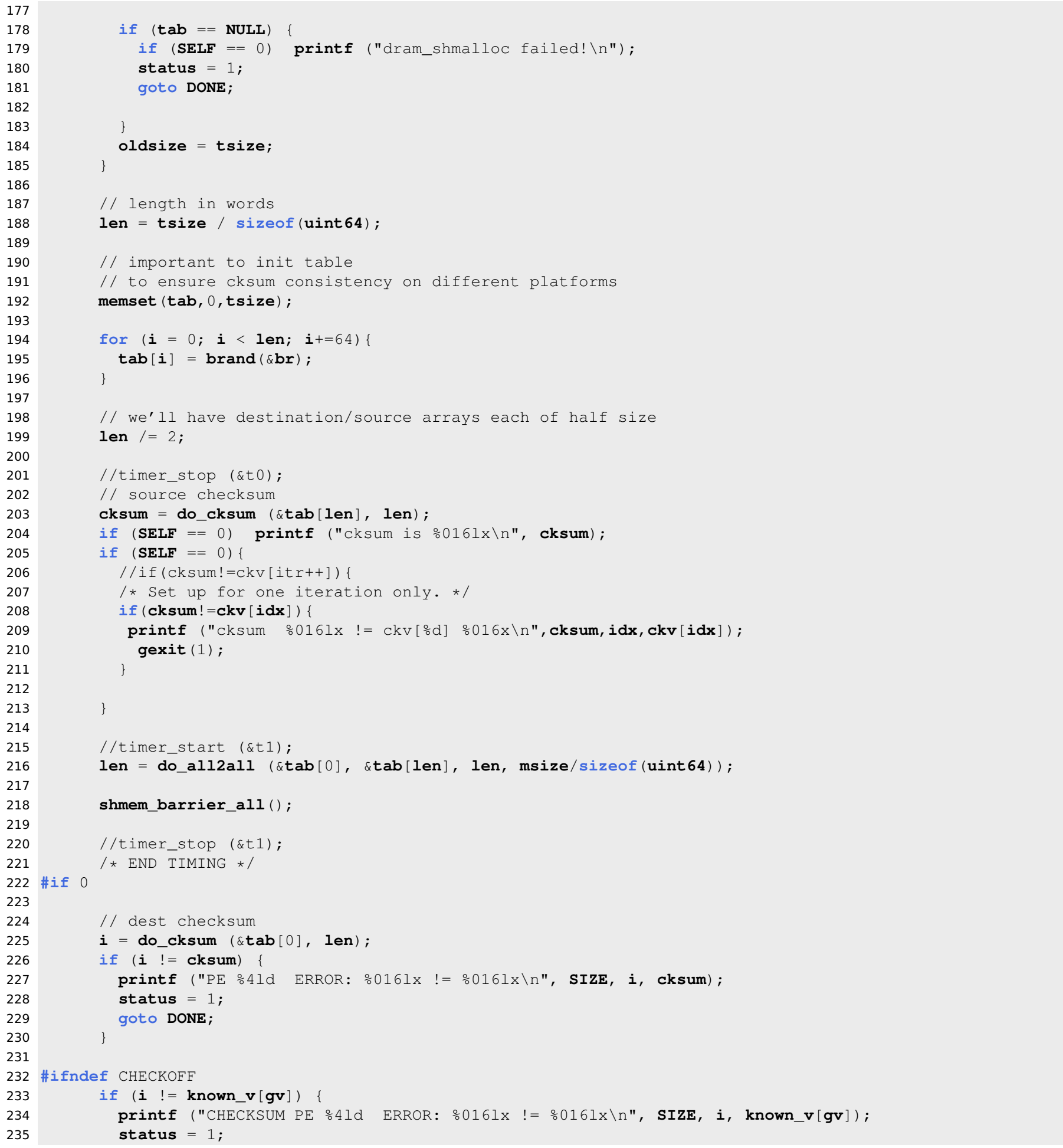




\section{E EXAMPLES}

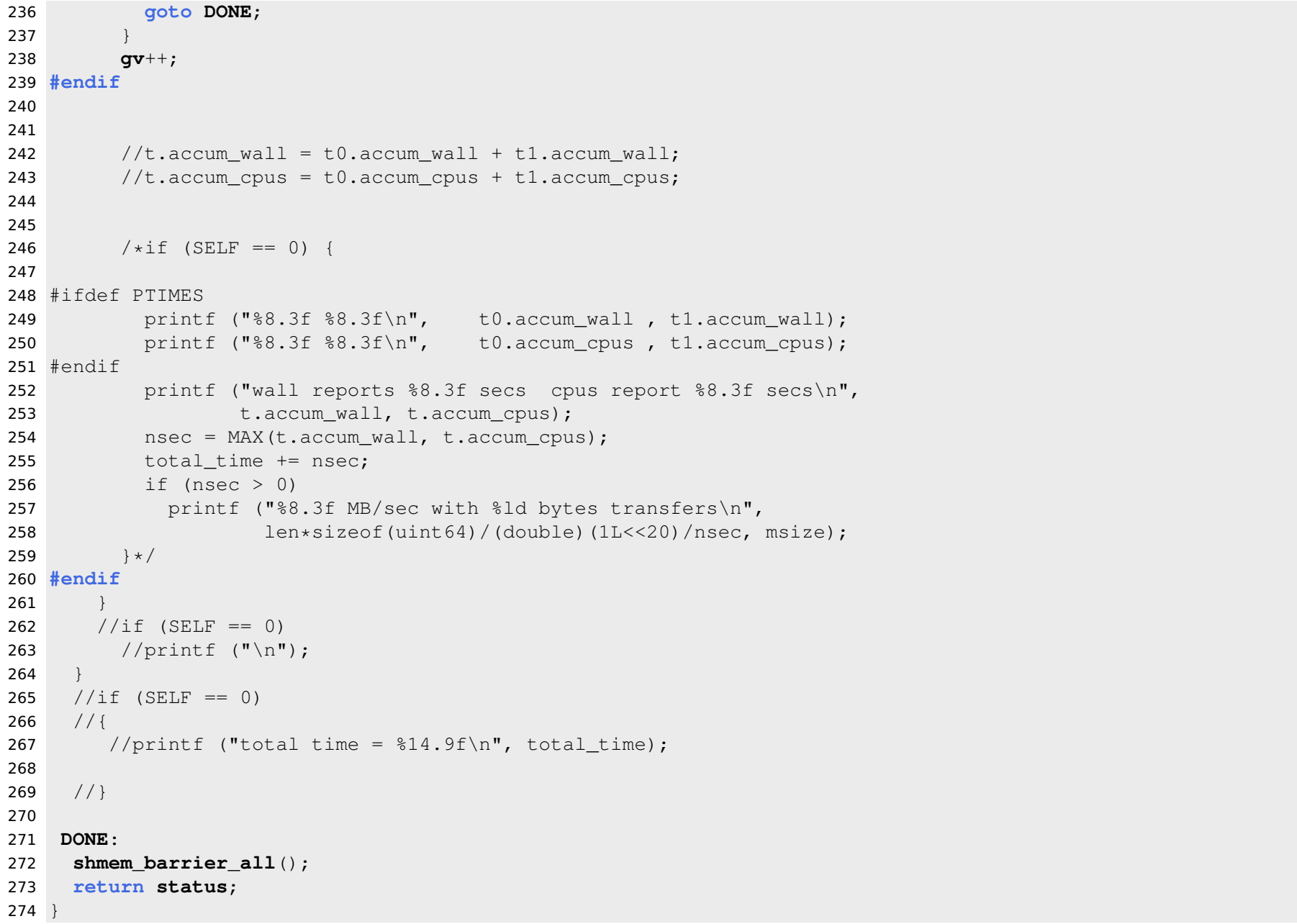


Listing 9: Program that implements an ALL-2-ALL (subs)

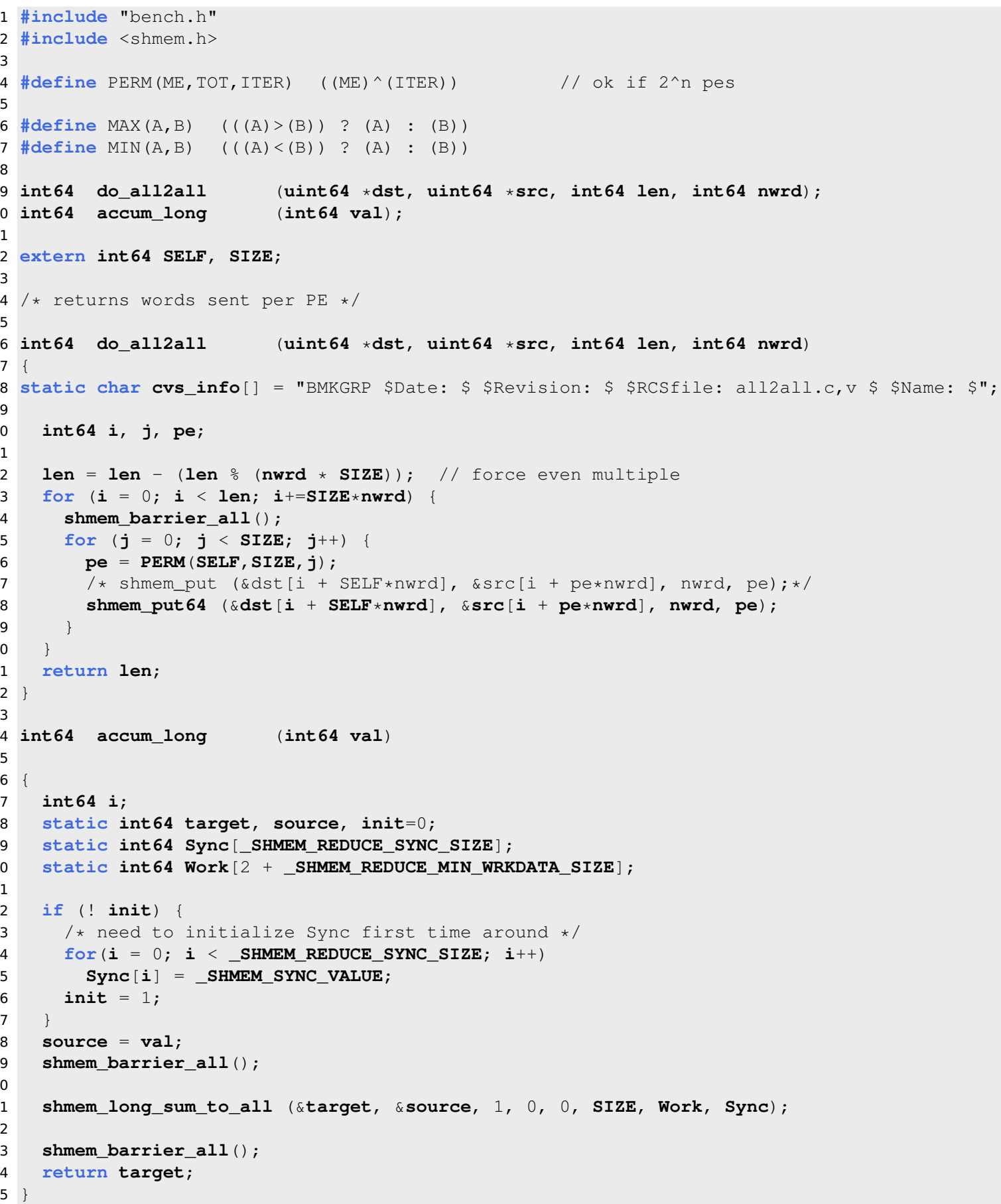




\section{Listing 10: Program that computes Pi}

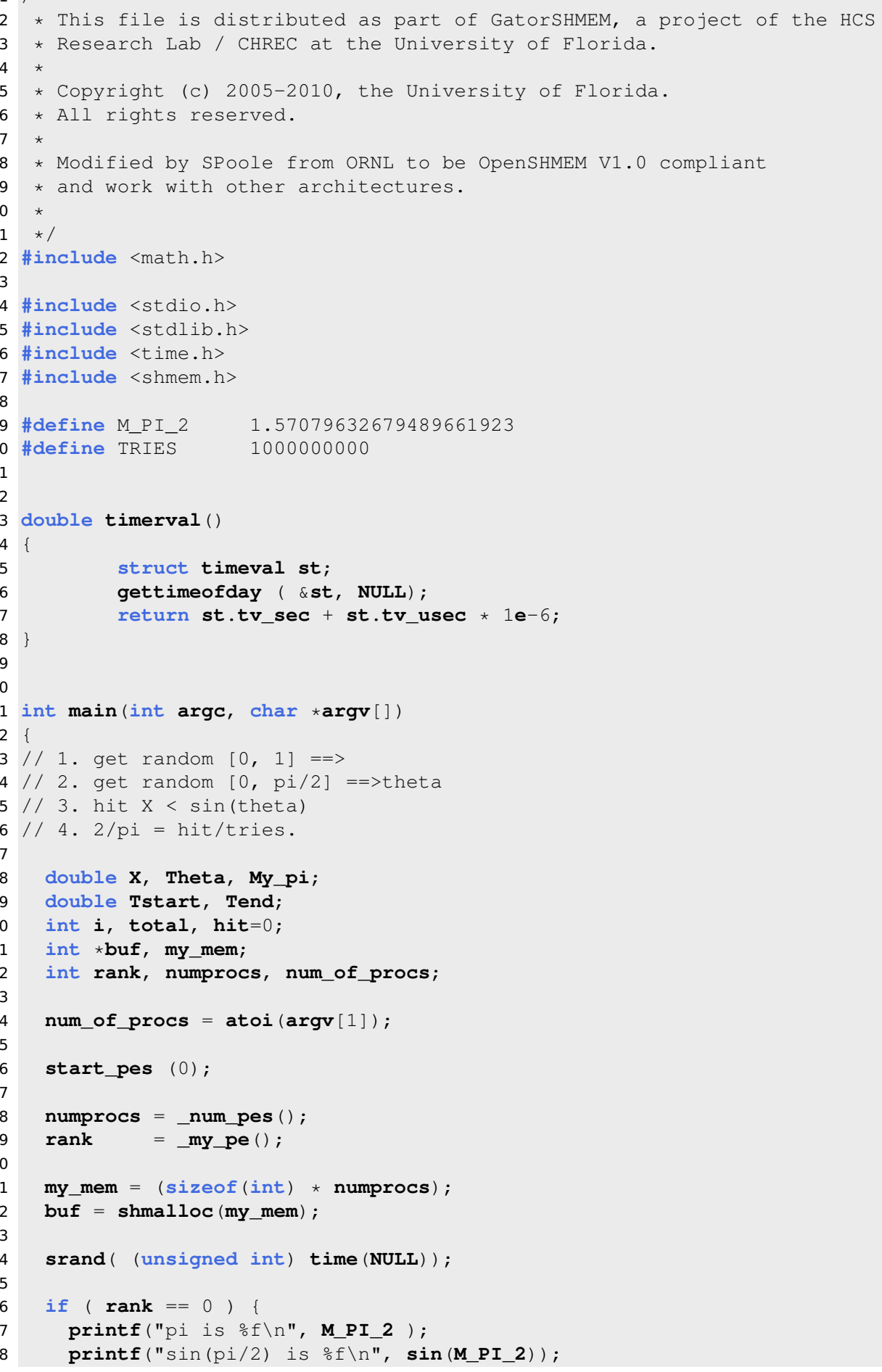




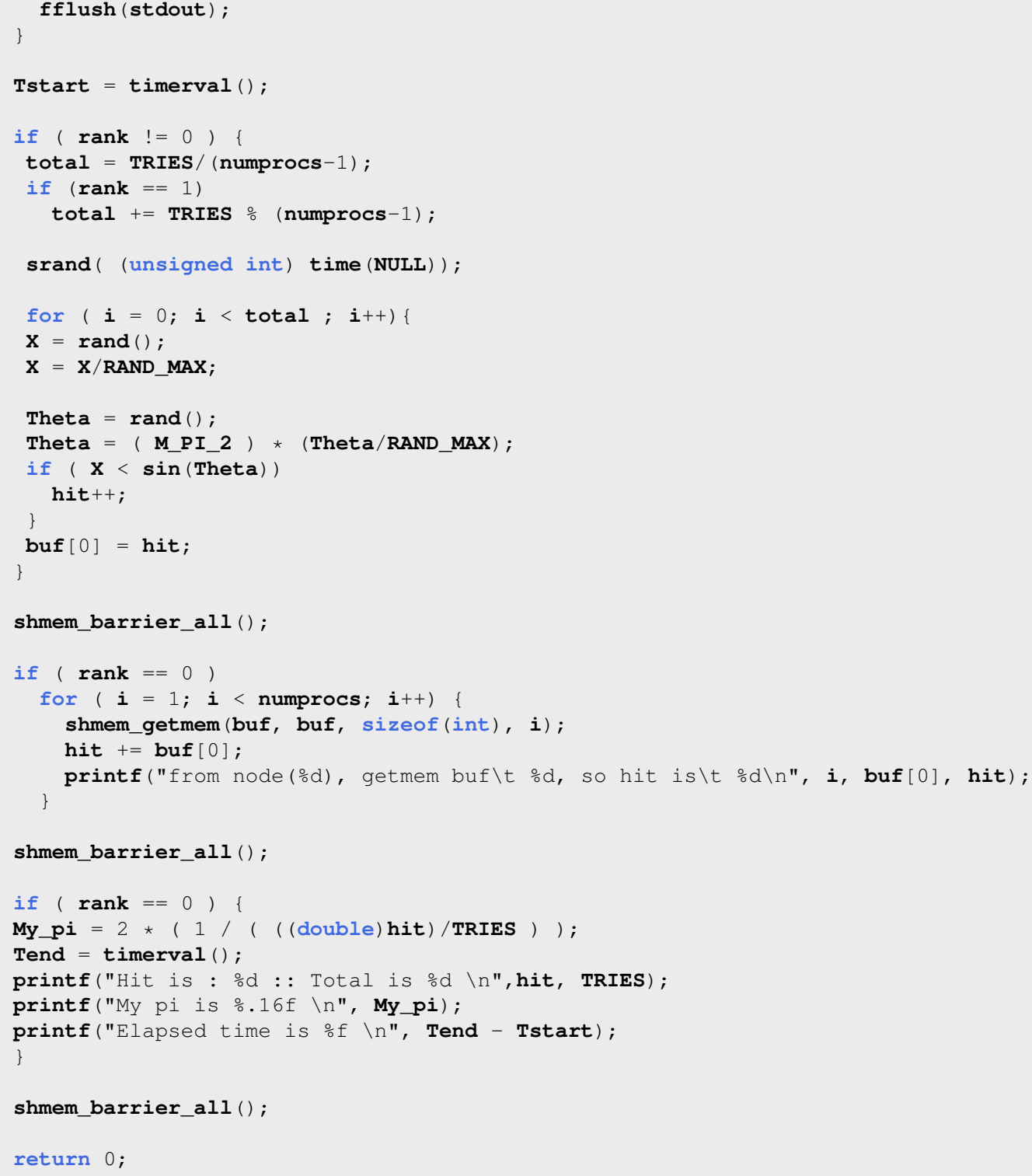




\section{E.2 Fortran Language Examples}

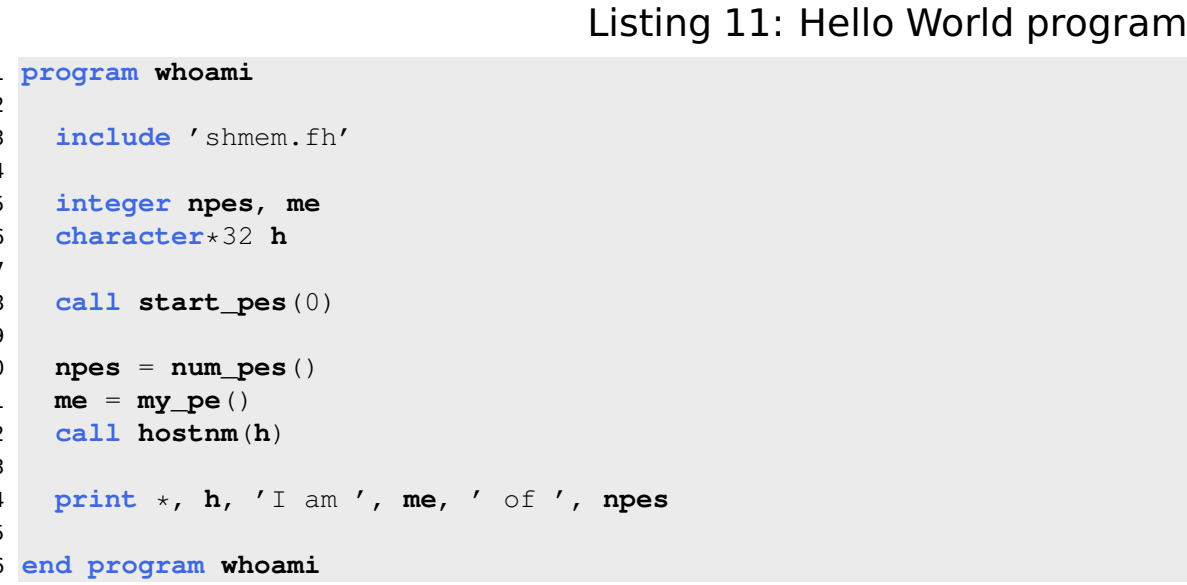




\section{F Glossary}

\section{F.1 OpenSHMEM Concepts}

processing element One of the processors involved in the execution of an OpenSHMEM application. Called "PE" for short.

PE number Integer value used to identify a processing element.

active set of PEs The group of PEs involved in the execution of a collective operation.

OpenSHMEM program Program that makes use of routines in the OpenSHMEM library.

put operation An operation that places data on a remote processing element.

get operation An operation that retrieves data from a remote processing element.

remote pointer $A$ pointer that allows to directly reference a data object that is stored in a remote processing element.

reduction Performs an associative binary operation across a set of values on multiple PEs.

broadcast A collective operation that places a data object available on a "root" PE, onto all other PEs in the broadcast's active set.

barrier A collective synchronization mechanism. PEs in the active set cannot leave the barrier routine until all of those PEs have reached the barrier. 


\title{
F.2 Data Terminology
}

\author{
cache Intermediate and transparent \\ store used to speed up future \\ requests. \\ symmetric data object A local data object that has a \\ corresponding data object on \\ all other PEs with the same \\ length, type and offset. \\ symmetric heap Special memory region, with \\ possibly different starting \\ address on each PE, in which \\ dynamically created \\ symmetric data objects are \\ stored. Objects in this region \\ have the same offset on every \\ PE with respect to the region's \\ starting address. \\ environment variable Set of named values, inherited \\ from execution/launch, that \\ will affect how programs \\ behave. \\ strided-data A special type of array in which \\ elements are separated by a \\ specific number (non-unit) of \\ memory locations.
}

\section{F.3 Implementation Terminology}

atomic operation An operation that guarantees that the resource being accessed will not be modified by another process until the operation is completed.

data latency The period of time that starts when a processing element initiates a transfer of data and ends when the processing element is able to make use of the data. 
overhead Any combination of network latency, memory bandwidth or computation time required to perform a communication operation.

mutual exclusion Mechanism used to avoid the simultaneous use of a shared resource.

lock Synchronization mechanism that limits access to a given resource.

undefined behavior A behavior not defined by the OpenSHMEM specification. See the Undefined Behavior section for more information about undefined behavior in OpenSHMEM . 


\section{References}

[1] ARMCI website,

http: / / www . emsl.pnl.gov/docs/parsoft/armci/.

[2] GASNet specification,

http://gasnet.cs.berkeley.edu/.

[3] GPSHMEM, http://www.scl.ameslab.gov/Projects/GP SHMEM/GP SHMEM. html.

[4] PGAS forum, http: //www.pgas.org/.

[5] TurboSHMEM, http://da.nieltiggemann.de/science/sc/turboshmem/.

[6] HPC Tools group at the University of Houston. Commonly used function calls in the OpenSHMEM library for $\mathrm{C} / \mathrm{C}++$ and FORTRAN, http://www.openshmem.org/wiki/index.php/Documentation:Tutorials.

[7] Krzysztof Parzyszek, Ricky A. Kendall, and Robyn R. Lutz. Generalized portable SHMEM library for High Performance Computing. lowa State University, 2003.

[8] Stephen W. Poole and Galen M. Shipman. Open-SHMEM: Towards a unified RMA model, 2000.

[9] Hongzhang Shan and Jaswinder Pal Singh. A Comparison of MPI, SHMEM and cachecoherent shared address space programming models on a tightly-coupled multiprocessors (sic.). International Journal of Parallel Programming, 29(3):283-318, June 2001.

[10] Tim Stitt. An introduction to the Partitioned Global Address Space (PGAS) programming model,

http://cnx.org/content/m20649/latest/.

[11] K. Yelick. Performance and productivity opportunities using global address space programming models, 2000. 